\title{
A new method to distinguish hadronically decaying boosted $Z$ bosons from $W$ bosons using the ATLAS detector
}

\author{
ATLAS Collaboration ${ }^{\star}$ \\ CERN, 1211 Geneva 23, Switzerland
}

Received: 17 September 2015 / Accepted: 8 April 2016/ Published online: 28 April 2016

(C) CERN for the benefit of the ATLAS collaboration 2016. This article is published with open access at Springerlink.com

\begin{abstract}
The distribution of particles inside hadronic jets produced in the decay of boosted $W$ and $Z$ bosons can be used to discriminate such jets from the continuum background. Given that a jet has been identified as likely resulting from the hadronic decay of a boosted $W$ or $Z$ boson, this paper presents a technique for further differentiating $Z$ bosons from $W$ bosons. The variables used are jet mass, jet charge, and a $b$-tagging discriminant. A likelihood tagger is constructed from these variables and tested in the simulation of $W^{\prime} \rightarrow W Z$ for bosons in the transverse momentum range $200 \mathrm{GeV}<p_{\mathrm{T}}<400 \mathrm{GeV}$ in $\sqrt{s}=8 \mathrm{TeV}$ $p p$ collisions with the ATLAS detector at the LHC. For Zboson tagging efficiencies of $\epsilon_{Z}=90,50$, and $10 \%$, one can achieve $W^{+}$-boson tagging rejection factors $\left(1 / \epsilon_{W^{+}}\right)$of $1.7,8.3$ and 1000 , respectively. It is not possible to measure these efficiencies in the data due to the lack of a pure sample of high $p_{\mathrm{T}}$, hadronically decaying $Z$ bosons. However, the modelling of the tagger inputs for boosted $W$ bosons is studied in data using a $t \bar{t}$-enriched sample of events in $20.3 \mathrm{fb}^{-1}$ of data at $\sqrt{s}=8 \mathrm{TeV}$. The inputs are well modelled within uncertainties, which builds confidence in the expected tagger performance.
\end{abstract}

\section{Introduction}

Processes involving the production and decay of $W$ and $Z$ bosons provide benchmarks for testing the Standard Model (SM), as well as probes of physics beyond the SM (BSM). Since the cross section for the direct strong production of events with multiple jets (QCD multijets) at the Large Hadron Collider (LHC) is much larger than for $W$ and $Z$ boson production, it is usually the case that the leptonic decays of bosons must be used to reduce the overwhelming background. However, when the momentum $p^{V}$ of a boson $V$ is comparable with its mass, $m_{V}$, the spatial proximity of the decay products provides a new set of tools that can be

^e-mail: atlas.publications@cern.ch used to distinguish between jets from hadronic boson decays and jets originating from QCD multijet backgrounds. In particular, since the angle between the decay products of a boson $V$ scales with $2 m_{V} / p^{V}$, for large $p^{V}$, jet substructure techniques become powerful tools. This leads to a tradeoff between using relatively pure leptonic decays and highbranching-ratio hadronic decays. In some BSM theories, new particles similar to $W / Z$ bosons do not couple directly to leptons, so searching for hadronic decays of heavy particles is essential.

Jet substructure techniques developed to distinguish hadronically decaying $W$ and $Z$ bosons from QCD multijet background processes have become increasingly sophisticated. A recent review is given in Ref. [1]. Both ATLAS [2] and CMS [3] have performed detailed comparisons of the various tagging variables and jet-grooming techniques with the overall conclusion that large QCD multijet suppression factors ${ }^{1}$ are possible while maintaining acceptable levels of boson tagging efficiency. Given a $W / Z$-boson tagger, a natural next step is to distinguish boson types.

There are several important possible applications of a boson-type tagger at the LHC. First, a type tagger could enhance the SM physics program with $W$ and $Z$ bosons in the final state. Measurements of this kind include the determination of the cross sections for $V+$ jets, $V V$, and $t \bar{t}+V$. Another important use of a boson-type tagger is in searches for flavour-changing neutral currents (FCNC). Due to the Glashow-Iliopoulos-Maiani (GIM) mechanism [4], FCNC processes in the SM are highly suppressed. Many models of new physics predict large enhancements to such processes. Both ATLAS and CMS have performed searches for FCNC [5,6] of the form $t \rightarrow Z q$ in the leptonic channels, but these could be extended by utilizing the hadronic $Z$ decays as well. FCNC processes mediated by a leptophobic $Z^{\prime}$ such as $t \rightarrow Z^{\prime} q$ may be detected only via hadronic type-tagging methods. A third use of a boson-type tagger is to categorize the properties of new physics, if discovered at the LHC.

$\overline{{ }^{1} \mathcal{O}(1 \%) \text { QCD }}$ multijet efficiency at $50 \%$ signal efficiency. 
For instance, if a new boson were discovered as a hadronic resonance, a boson-type tagger could potentially distinguish a $W^{\prime}(\rightarrow q q)$ from a $Z^{\prime}(\rightarrow q q)$ (where mass alone may not be useful). This is especially relevant for leptophobic new bosons, which could not be distinguished using leptonic decays.

Labelling jets as originating from a $W$ or $Z$ boson is less ambiguous than quark/gluon labelling. A $W$ boson can radiate a $Z$ boson, just like a quark can radiate a gluon, but this is heavily suppressed for the former and not for the latter. The radiation pattern of jets from $W$ - and $Z$-bosons is less topology dependent because it is largely independent of the other radiation in the event as $W$ and $Z$ bosons are colour singlets. Aside from the production cross section and subtle differences in differential decay distributions, the only features that distinguish between $W$ and $Z$ bosons are their mass, charge, and branching ratios. Experimentally, this means that the only variables that are useful in discriminating between hadronic decays of $W$ and $Z$ bosons are those which are sensitive to these properties. The three variables used in the analysis presented here are jet mass, sensitive to the boson mass, jet charge, sensitive to the boson charge, and a $b$ tagging discriminant which is sensitive to the heavy-flavour decay branching fractions of the bosons. The application of a boson-type tagger in practice will be accompanied by the prior use of a boson tagger (to reject QCD multijet processes). The type-tagger variables are largely independent of typical boson-tagger discriminants like $n$-subjettiness [7], which rely on the two-prong hard structure of both the $W$ and $Z$ decays. ${ }^{2}$

This paper introduces a jet tagging method to distinguish between hadronically decay $W$ and $Z$ bosons at the LHC, and documents its performance with the ATLAS detector at $\sqrt{s}=8 \mathrm{TeV}$. The paper is organized as follows. Section 2 describes the simulated datasets used in constructing and evaluating the boson-type tagger. Following a discussion of the differences between the properties of $W$ and $Z$ bosons in Sect. 3, Sect. 4 defines the three discriminating variables. The construction and performance of the tagger are detailed in Sect. 5 and the sensitivity to systematic uncertainties is described in Sect. 6. The input variables are studied in a dataset enriched in boosted $W$ bosons in Sect. 7. The paper ends with a discussion of possible uses of the tagger in Sect. 8 and conclusions in Sect. 9.

\section{Datasets}

Two sets of Monte Carlo (MC) simulations are generated, one to study the tagger's $W$ versus $Z$ performance and the other to compare the tagger inputs for $W$ bosons with the data. Simulations of hypothetical $W^{\prime} \rightarrow W Z$ production and

\footnotetext{
${ }^{2}$ See Sect. A. for details.
}

decay provide a copious source of boosted $W$ and $Z$ bosons whose $p_{\mathrm{T}}$ scale is set by the mass of the $W^{\prime}$ boson. Such events are used to construct a tagger to separate hadronically decaying boosted $W$ and $Z$ bosons, as well as to evaluate its performance. It is not possible to measure the performance directly in the data due to the lack of a pure sample of boosted, hadronically decaying $Z$ bosons, but the modelling of the tagger inputs can be studied using hadronically decaying $W$ bosons from $t \bar{t}$ events in the data.

A simulated sample of $W^{\prime}$ bosons is generated with PYTHIA 8.160 [8] using the leading-order parton distribution function set (PDF) MSTW2 008 [9,10] and the AU2 [11] set of tunable parameters (tune) for the underlying event. The baseline samples use PYTHIA for the $2 \rightarrow 2$ matrix element calculation, as well as $p_{\mathrm{T}}$-ordered parton showers [12] and the Lund string model [13] for hadronization. Additional samples are produced with HERWIG++ [14], which uses angular ordering of the parton showers [15], a cluster model for hadronization [16], as well as the EE3 [17] underlyingevent tune. The $W$ ' differs from the SM $W$ boson only in its mass and the branching ratio $W^{\prime} \rightarrow W Z$ is set to $100 \%$. The $W$ and $Z$ bosons are produced with a mixture of polarizations, but the longitudinal polarization state dominates because $m_{W}, m_{Z} \ll m_{W^{\prime}}$. In order to remove artifacts in the $p_{\mathrm{T}}$ distributions of the $W$ and $Z$ bosons due to the generation of $W^{\prime}$ particles with discrete masses, the $p_{\mathrm{T}}^{V}$ spectra are re-weighted to be uniform in the range $200 \mathrm{GeV}<p_{\mathrm{T}}^{V}<400 \mathrm{GeV}$. As is discussed in Sect. 1, for $p_{\mathrm{T}}>200 \mathrm{GeV}$, a jet with large radius is expected to capture most of the $W$ or $Z$ boson decay products. The range is truncated to $p_{\mathrm{T}}<400 \mathrm{GeV}$ because hadronically decaying $W$ bosons can be probed with data in this $p_{\mathrm{T}}$ range; there are too few events in the $8 \mathrm{TeV}$ dataset for $p_{\mathrm{T}}>400 \mathrm{GeV}$.

Top-quark pair production is simulated using the next-toleading-order (NLO) generator POWHEG-BOX [18-20] with the NLO PDF set CT10 [10] and parton showering from PYTHIA 6 [21]. The single-top ( $s-, t-$, and $W t$-channel) backgrounds are modelled with POWHEG-BOX and PYTHIA 6 , as for the nominal $t \bar{t}$ simulation. The PDF set CT1 0 f 4 [9] is used for the $t$-channel and CT10 is used for the $s$-and $W t$-channels. For the $W t$-channel, the 'inclusive Diagram Removal' (DR) scheme is used for overlap with $t \bar{t}$ [22]. The $W+$ jets and $Z+$ jets backgrounds are modelled with ALPGEN 2.1.4 [23], PYTHIA 6 and the CTEQ6L1 PDF set [24]. Dibosons are generated with HERWIG 6 . 520.2 [25] using the CTEQ6L1 PDF set and the AUET2 tune [26]. Version 6.426 is used everywhere for PYTHIA 6, with the Perugia2011C tune [27].

Events are processed with a full simulation of the ATLAS detector and trigger [28] based on the Geant 4 [29] toolkit, and reconstructed using the same software as for the experimental data. The average number of additional $p p$ collisions per bunch crossing (pileup interactions) was 20.7 over the full 
2012 run. The effects of pileup are modelled by adding multiple minimum-bias events, which are simulated with PYTHIA 8.160 , to the generated hard-scatter events. The distribution of the number of interactions is then weighted to reflect the pileup distribution in the 2012 data. A sample of $W$ bosons is selected from data taken in 2012 at centre-of-mass energy of $\sqrt{s}=8 \mathrm{TeV}$ from $t \bar{t}$ candidates as described in Sect. 7 .

\section{Distinguishing a $Z$ boson from a $W$ boson}

Decays of $W$ or $Z$ bosons are characterized by the boson's mass and coupling to fermions. The mass difference between the $W$ and $Z$ boson is about $10 \mathrm{GeV}$ and if produced from a hard scatter or the decay of a heavy enough resonance, both bosons are produced nearly on-shell since the width $\Gamma_{V}=2.1$ (2.5) $\mathrm{GeV}$ is much less than the mass $m_{V}=80.4$ (91.2) GeV for $W(Z)$ bosons [30]. The Breit-Wigner resonance curves for $W$ and $Z$ bosons are shown in Fig. 1a. The separation between the curves is a theoretical limit on how well mass-sensitive variables can distinguish between $W$ and $Z$ bosons. For hadronic boson decays, the mass peaks measured with jets are broader. This is because the jet-clustering algorithm for final-state hadrons loses parti- cles at large angles to the jet axis and includes extra particles from the underlying event and pileup.

The generic coupling of a boson $V$ to fermions is given by $g_{\mathrm{V}} \gamma_{\mu}\left[c_{\mathrm{V}}-c_{\mathrm{A}} \gamma_{5}\right]$, where $g_{\mathrm{V}}$ is a boson-dependent overall coupling strength, and $c_{\mathrm{V}}$ and $c_{\mathrm{A}}$ are the vector and axial-vector couplings, respectively. The $W$ boson couples only to left-handed fermions so $c_{\mathrm{V}}=c_{\mathrm{A}}=1$ with $g_{W} \propto$ $k N_{\mathrm{C}} G_{\mathrm{F}} m_{W}^{3}\left|V_{i j}\right|^{2}$, where $G_{\mathrm{F}}$ is the Fermi coupling constant, $V_{i j}$ is a Cabibbo-Kobayashi-Maskawa (CKM) matrix element [31,32], $k$ represents higher-order corrections, and $N_{\mathrm{C}}=3$ for the three colours of quarks and $N_{\mathrm{C}}=1$ for leptons. The CKM matrix is nearly diagonal so $W^{+} \rightarrow u \bar{d}$ and $W^{+} \rightarrow c \bar{s}$ are the dominant decay modes. Small off-diagonal elements contribute to the other possible decay modes, and the overall hadronic branching ratios are approximately $50 \%$ for $W \rightarrow c X$ and $50 \%$ for $W \rightarrow$ light-quark pairs. The $W$ boson has electric charge \pm 1 in units of the electron charge, so by conservation of charge, its decay products have the same net charge. The scalar sum of the charge of all the final-state hadrons originating from a $W$ boson decay is not infrared safe (directly sensitive to the non-zero detection threshold), so there are limits to the performance of charge tagging dictated by the energy threshold placed on charged particles in the event reconstruction.
Fig. 1 a Breit-Wigner resonances for the $W$ (red) and $Z$ (blue) bosons, $\mathbf{b}$ angular distribution of the decay products of transversely polarized $W / Z$ bosons with respect to the spin direction in the boson rest frame, $\mathbf{c}$ hadronic branching fractions of the $W^{+}$ boson, and $\mathbf{d}$ of the $Z$ boson. In c, d, light stands for decay modes not involving $\mathbf{b}, \mathbf{c}$ quarks

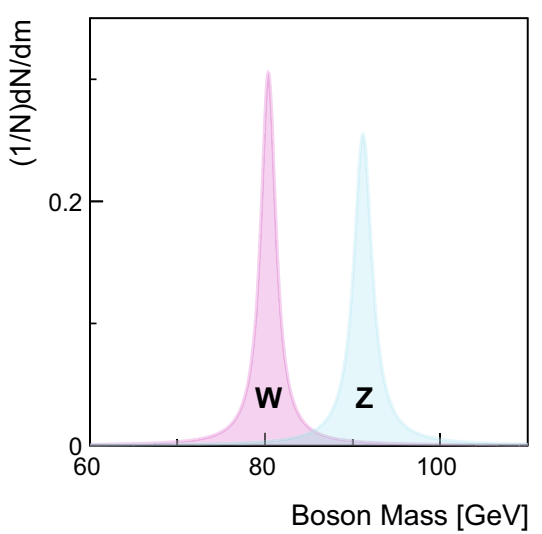

(a)

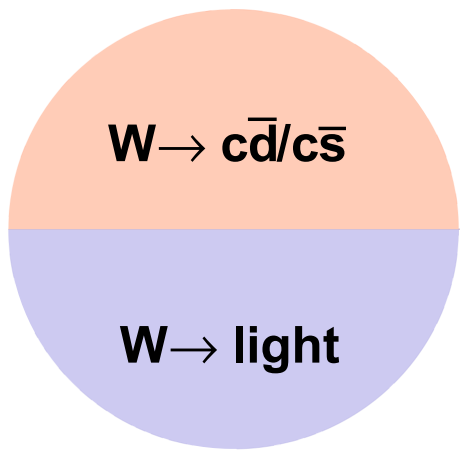

(c)

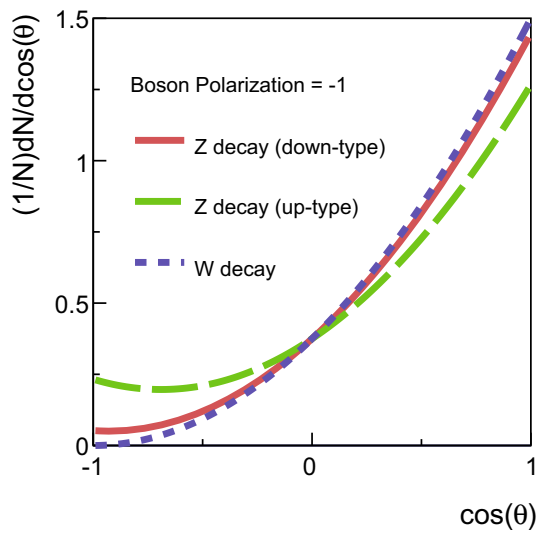

(b)

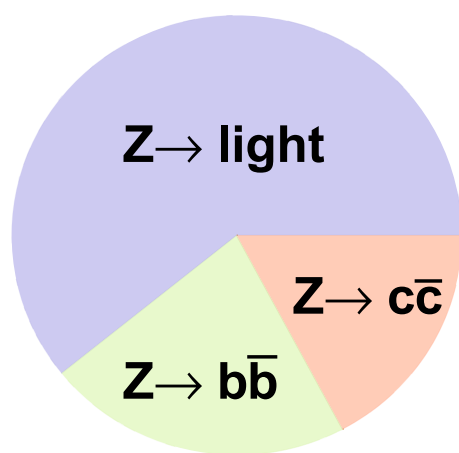

(d) 
In contrast to $W$ boson decays, $Z$ bosons decay to both the left- and right-handed fermions. The partial width for $Z \rightarrow$ $f \bar{f}$ is proportional to $k N_{\mathrm{C}} G_{\mathrm{F}} m_{Z}^{3}\left[c_{\mathrm{V}}^{2}+c_{\mathrm{A}}^{2}\right]$. The factors $c_{\mathrm{V}}$ and $c_{\mathrm{A}}$ are slightly different for up- and down-type fermions. The $b \bar{b}$ branching ratio is $22 \%$, the $c \bar{c}$ branching ratio is $17 \%$ and the sum of the remaining branching ratios is $61 \%$. W boson decays to $b$-quarks are highly suppressed by the small CKM matrix elements $V_{c b}$ and $V_{u b}$, so that identifying $b$ hadron decays associated with a hadronically decaying boson is a powerful discriminating tool. Branching ratios are plotted in Fig. 1d for $Z$ decays to light quarks, $c$-quarks, and $b$ quarks, and in Fig. 1c for the $W$ boson decays to light quarks and $c$-quarks.

Since the coupling structure is not identical for $W$ and $Z$ bosons, the total decay rates differ, and the angular distributions of the decay products also differ slightly. However, even at parton level without any combinatoric noise, the differences in the angular distributions are subtle. There is no difference for the two bosons with longitudinal polarization because the distributions for right- and left-handed fermions are the same. The distributions are different for right- and left-handed fermions for transversely polarized $W$ and $Z$ bosons, as shown in Fig. 1b. The relative contribution of left- and right-handed components for the $Z$ decays depends on the quark flavour; for up-type quarks the relative contribution from right-handed fermions is $15 \%$ while it is only $3 \%$ for down-type quarks. In $t \bar{t}$ decays, the fraction of longitudinally polarized $W$ bosons (ignoring the $b$-quark mass) is $m_{t}^{2} /\left(m_{t}^{2}+2 m_{W}^{2}\right) \sim 0.7$. In contrast, the boson is mostly transversely polarized in inclusive $V+$ jets events. Any discrimination shown in Fig. 1b is diluted by the longitudinal polarization, combinatorics, non-perturbative effects, and detector reconstruction, so angular distributions are not considered further in this paper. ${ }^{3}$

\section{Definitions of reconstructed objects}

ATLAS is a multi-purpose particle detector [33] with nearly $4 \pi$ coverage in solid angle. ${ }^{4}$ The energy of the hadronic

\footnotetext{
3 The impact of polarization on distinguishing boosted $W$ boson jets from QCD multijets has been studied in Ref. [3]. There are small differences in performance between transversely and longitudinally polarized bosons, but any differences are less relevant for $W$ versus $Z$ tagging where the angular distributions are identical for longitudinally polarized bosons and only slightly differ for transversely polarized bosons.

4 ATLAS uses a right-handed coordinate system with its origin at the nominal interaction point (IP) in the centre of the detector and the $z$-axis along the beam pipe. The $x$-axis points from the IP to the centre of the LHC ring, and the $y$-axis points upward. Polar coordinates $(r, \phi)$ are used in the transverse plane, $\phi$ being the azimuthal angle around the beam pipe. The pseudorapidity is defined in terms of the polar angle $\theta$ as $\eta=-\ln \tan (\theta / 2)$. Transverse momentum and energy are defined in the $x-y$ plane as $p_{\mathrm{T}}=p \cdot \sin (\theta)$ and $E_{\mathrm{T}}=E \cdot \sin (\theta)$.
}

decay products of boosted bosons is measured by a system of calorimeters. The electromagnetic calorimeter consists of a $\mathrm{Pb} /$ liquid-argon sampling calorimeter split into barrel $(|\eta|$ $<1.5)$ and endcap $(1.5<|\eta|<3.2)$ sections. The hadronic calorimetry is provided by a barrel steel/scintillating-tile calorimeter $(|\eta|<1.7)$ and two endcap $\mathrm{Cu} /$ liquid-argon sections $(1.5<|\eta|<3.2)$. Finally, the forward region

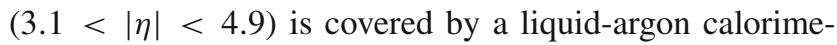
ter with $\mathrm{Cu}(\mathrm{W})$ absorber in the electromagnetic (hadronic) section. Energy depositions are grouped into topological calorimeter-cell clusters [34] and then calibrated using the local cluster weighting algorithm [35,36]. Jets are formed from clusters using two different jet algorithms. Small-radius jets are built with the anti- $k_{t}$ algorithm with jet radius parameter $R=0.4$ [37]. Large-radius jets are formed using the anti- $k_{t}$ algorithm with $R=1.0$ and then trimmed [38] by re-clustering the jet constituents with the $k_{t}$ algorithm using $R=0.3$ and removing the constituents with $p_{\mathrm{T}}$ less than $5 \%$ of the original jet $p_{\mathrm{T}}$. Both the small- and large-radius jets are further calibrated to account for the residual detector response effects. For small-radius jets, this is a $p_{\mathrm{T}^{-}}$and $\eta$-dependent energy calibration, plus a correction to mitigate the contribution from additional $p p$ collisions and to suppress jets from these additional collisions [39]. In addition to $p_{\mathrm{T}}$ - and $\eta$-dependent energy corrections, large-radius jets $J$ have a calibrated jet mass:

$m_{J}^{2}=\left(\sum_{j \in J} E_{j}\right)^{2}-\left(\sum_{j \in J} \vec{p}_{j}\right)^{2}$,

where $E_{j}$ is the energy of cluster $j$ and $\vec{p}_{j}$ is a vector with magnitude $E_{j}$ and direction $\left(\phi_{j}, \eta_{j}\right)$. The jet mass calibration depends on the calibrated jet energy and on the jet $\eta$ [45]. When a $W$ or $Z$ boson is produced with large enough momentum, its decay products are collimated. When $2 m_{V} / p^{V} \sim 1$, an $R=1.0$ trimmed jet captures a large fraction of the decay products and the jet-mass scale is set by $m_{V}$. Since the $W$ and $Z$ boson masses differ by about $10 \mathrm{GeV}$, the jet mass can be used to discriminate between these two particles. The distributions of the boson masses and jet masses for hadronically decaying $W$ and $Z$ bosons are shown in Fig. 2 . The particle-level ('truth') jet mass is constructed from stable particles in the MC simulation $(c \tau>10 \mathrm{~mm})$, excluding neutrinos and muons, clustered with the same jet algorithm as for calorimeter-cell clusters. The QCD processes that govern the formation of stable particles from the $W$ and $Z$ decay products create a broad distribution of jet masses even without taking into account detector resolution. Constructing the jet mass from calorimeter-cell clusters further broadens the distribution. The jet-mass resolution (physical $\oplus$ detector) is large compared to the natural width of the $W$ and $Z$ bosons and comparable to the difference in their 


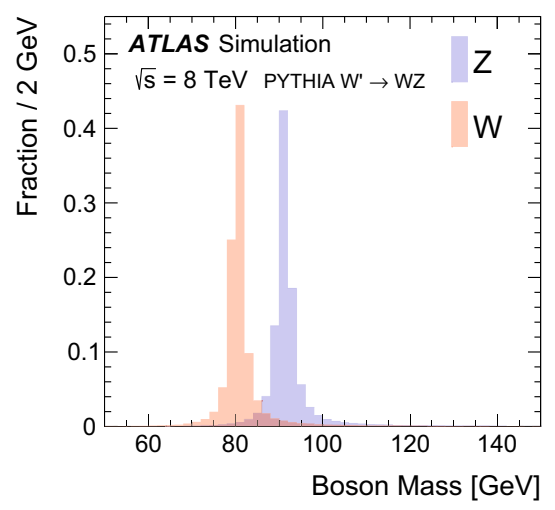

(a)

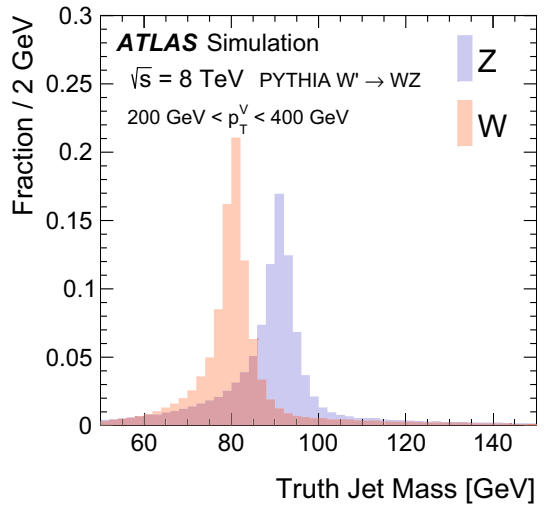

(b)

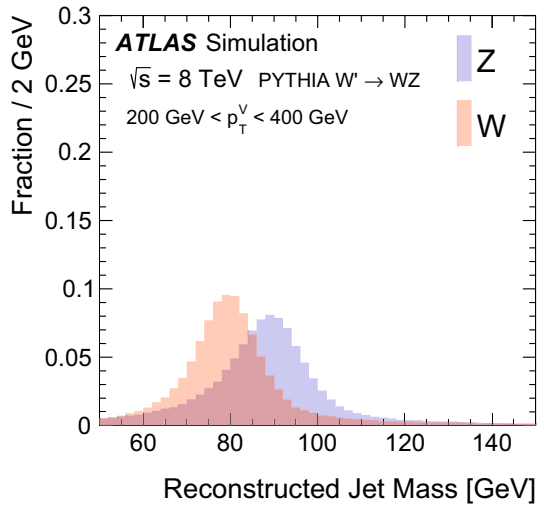

(c)

Fig. 2 a The boson mass at generator level, b 'truth' jet mass (at particle level) after parton fragmentation, and $\mathbf{c}$ reconstructed jet mass distributions. The left plot has a different vertical scale than the right two plots and also has no $p_{\mathrm{T}}$ requirement

masses. For example, the standard deviation of the detector resolution $\sigma\left(m^{\text {reco jet }} / \mathrm{m}^{\text {truth jet }}\right)$ is approximately $10 \%$. The jet-mass variable nevertheless has some discriminating power.

The momentum and electric charge of particles traversing the detector contain information about the charge of their parent boson. The tracks of charged particles are measured in a $2 \mathrm{~T}$ axial field generated by a solenoid magnet which surrounds the inner detector (ID) consisting of silicon pixels, silicon micro-strips, and a transition radiation tracking detector. Charged-particle tracks are reconstructed from all three ID technologies with a full coverage in $\phi,|\eta|<2.5$ and $p_{\mathrm{T}}>400 \mathrm{MeV}$. The charge $q$ of a track is determined as part of the reconstruction procedure, which uses a fit with five parameters: the transverse and longitudinal impact parameters, $\phi, \theta$, and $q / p$, where $p$ is the track momentum. To suppress the impact of pileup, tracks are required to originate from the primary collision vertex, which is defined as the vertex with the largest $\sum p_{\mathrm{T}}^{2}$ computed from associated tracks. Additionally, tracks must satisfy a very loose quality criterion for the track fit $\chi^{2}$ per degree of freedom, which must be less than three. Tracks are associated with jets using ghost association [40]. The charge of tracks associated with a jet is sensitive to the charge of the initiating parton. In order to minimize the fluctuations due to low- $p_{\text {T }}$ particles, the jet charge is calculated using a $p_{\mathrm{T}}$-weighting scheme [41]:

$Q_{J}=\frac{1}{\left(p_{\mathrm{T}, \mathrm{J}}\right)^{\kappa}} \sum_{i \in \text { Tracks }} q_{i} \times\left(p_{\mathrm{T}}^{i}\right)^{\kappa}$

where Tracks is the set of tracks with $p_{\mathrm{T}}>500 \mathrm{MeV}$ associated with jet $J, q_{i}$ is the charge (in units of the electron charge) determined from the curvature of track $i$ with associated $p_{\mathrm{T}}^{i}$, $\kappa$ is a free parameter, and $p_{\mathrm{T}, \mathrm{J}}$ is the transverse momentum of the jet measured in the calorimeter. The calorimeter energy is

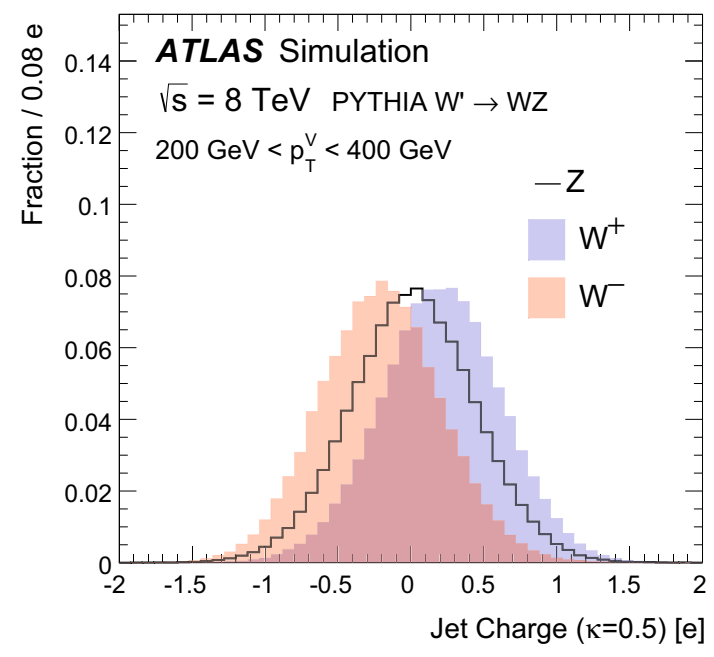

Fig. 3 The jet charge distribution for jets originating from $W^{ \pm}$and $Z$ bosons in simulated $W^{\prime}$ decays. Each distribution is normalized to unity. The parameter $\kappa$ controls the $p_{\mathrm{T}}$-weighting of the tracks in the jet charge sum

used in the denominator to determine $p_{\mathrm{T}}$ instead of the sum of track momenta to account for the contribution from neutral particles. Dedicated studies have shown that $\kappa=0.5$ is generally best for determining the charge of partons from the jets they produce [42]. The distributions of the jet charge for jets initiated by $W^{+}, W^{-}$and $Z$ bosons are shown in Fig. 3 . There is an observable separation between positive and negative $W$ bosons. The expected charge composition of a $W$ sample is process dependent. For example, there are more $W^{+}$than $W^{-}$bosons in inclusive $W^{\prime}$ production because of the initial charge asymmetry of quarks in the proton resulting in more $W^{\prime+}\left(\rightarrow W^{+} Z\right)$ than $W^{\prime-}\left(\rightarrow W^{-} Z\right)$. The discrimination between $Z$ bosons and a near even mixture of $W^{ \pm}$is greatly diminished with respect to e.g. $Z$ versus $W^{+}$. In that case charge sensitive variables are not very useful for the tag- 


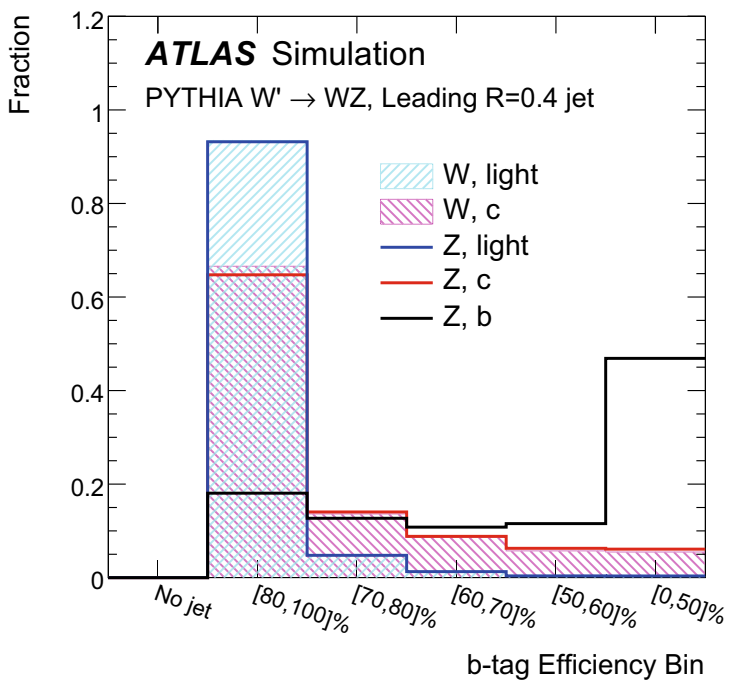

Fig. 4 The efficiency-binned MV1 distribution for small-radius jets associated with large-radius jets resulting from $W$ and $Z$ boson decays. The left (right) plot shows the leading (sub-leading) small-radius jet MV1 distribution. The bins correspond to exclusive regions of $b$-jet

ger and so all results are shown also without such variables. In a variety of physics processes, the charge of the hadronically decaying $W$ boson is known from other information in the event. For example, in searches for FCNC effects in $t \bar{t}$ events with one leptonically decaying $W$ boson, the charge of the lepton is opposite to the charge of the hadronically decaying $W$ boson. Henceforth, only $W^{+}$bosons are used for constructing the boson-type tagger; the results are the same for $W^{-}$bosons.

The tracks from charged particles can be used further to identify the decays of certain heavy-flavour quarks inside jets due to the long $b$-hadron lifetime. This is useful for bosontype tagging because the $Z$ boson couples to $b \bar{b}$ while decays of the $W$ boson to $b$-quarks are highly suppressed and can be neglected. ATLAS has commissioned a $b$-tagging algorithm called MV1 (defined in Refs. [43,44]) which combines information about track impact-parameter significance with the explicit reconstruction of displaced $b$ - and $c$-hadron decay vertices. The boson-type tagger presented here uses multiple bins of the MV1 distribution simultaneously. Five bins of MV1 are defined by $b$-tag efficiencies (probability to tag a $b$-quark jet as such) of $0-50,50-60,60-70,70-80$, and $80-100 \%$ as determined in simulated $t \bar{t}$ events. A lower $b$ tag efficiency leads to higher light-quark jet rejection. The five $b$-tagging efficiency bins are exclusive and MV1 is constructed as a likelihood with values mostly between zero and one (one means more like a $b$-jet). For example, a $100 \% b$ tagging efficiency corresponds to a threshold of MV1 $>0$ and an $80 \% b$-tagging efficiency corresponds to a threshold value of MV1 $>z$ for $z \ll 1$. The $80-100 \% b$-tag efficiency bin then corresponds to jets with an MV1 value between 0

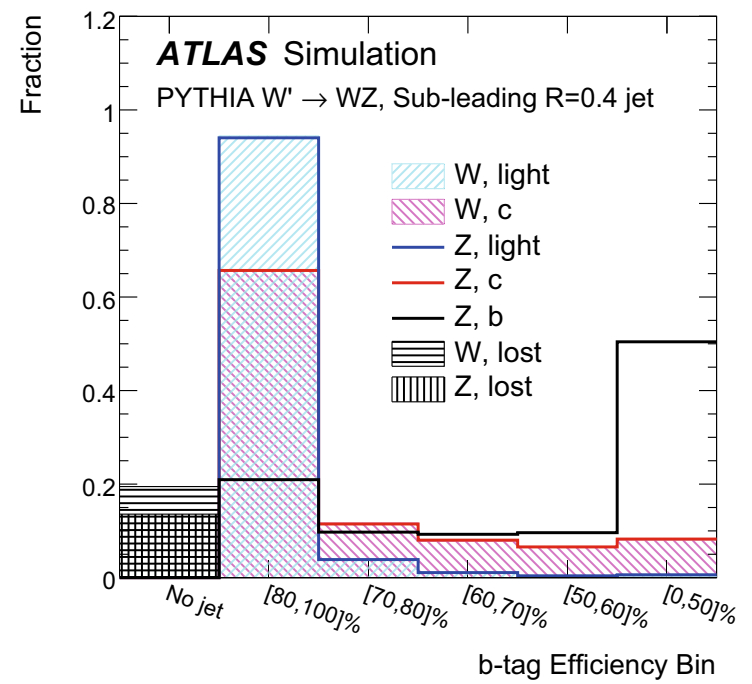

efficiency. As such, the bin content of the black line ( $b$-tagging for $b$ jets) should be proportional to the size of the efficiency window: about $50 \%$ for the rightmost bin, $10 \%$ for the three middle bins and $20 \%$ for the second bin

and $z$. Constructed in this way, the fraction of true $b$-jets inside an efficiency bin $x \%-y \%$ should be $(y-x) \%$.

Small-radius jets are matched to a large-radius jet by geometric matching ${ }^{5}(\Delta R<1.0)$. Of all such small-radius jets, the two leading ones are considered. There are thus 30 possible bins of combined MV1 when considering the leading and sub-leading matched small-radius jet. The number of bins is 25 from the $5 \times 5$ efficiency-binned MV1 distributions in addition to five more for the case in which there is no second small-radius jet matched to the large-radius jet. The distribution for the efficiency-binned MV1 variable for the leading and sub-leading matched small-radius jets is shown for $W$ and $Z$ bosons in Fig. 4. The flavour of a small-radius jet is defined as the type of the highest energy parton from the parton shower record within $\Delta R<0.4$. As expected, a clear factorization is seen in Fig. 4 - the MV1 value depends on the flavour of the small-radius jet and not the process that created it. This means that $c$-jets from $W$ decays have the same MV1 distribution as $c$-jets from $Z$ decays; the same is true for light jets. Small-radius jets originating from $b$-hadron decays tend to have a larger value of MV1, which means they fall in a lower efficiency bin. Small-radius jets not originating from $b$ or $c$-decays are called light jets and are strongly peaked in the most efficient bin of MV1. There is always one small-radius jet matched to the large-radius jet, but about $20 \%$ of the time there is no sub-leading small-radius jet with $p_{\mathrm{T}}>25 \mathrm{GeV}$

\footnotetext{
5 In the definition of jets, $R$ is the characteristic size in $(y, \phi)$ and the rapidity $y$ is used in the jet clustering procedure, whereas geometrical matching between reconstructed objects is performed using $(\Delta R)^{2}=$ $(\Delta \phi)^{2}+(\Delta \eta)^{2}$, where $\eta$ is the pseudorapidity.
} 


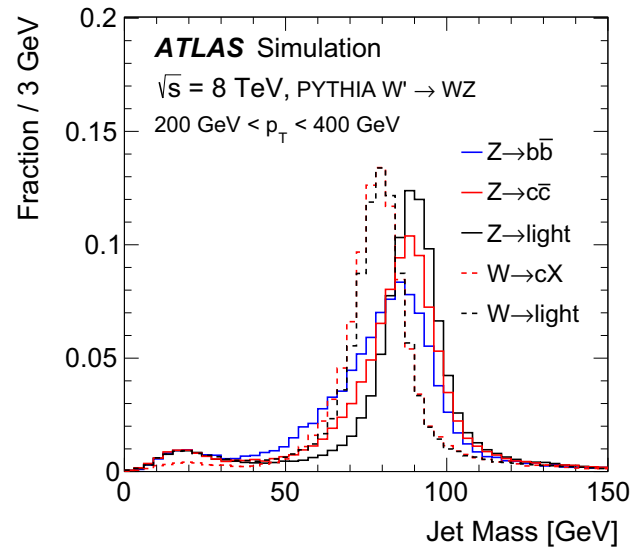

(a)

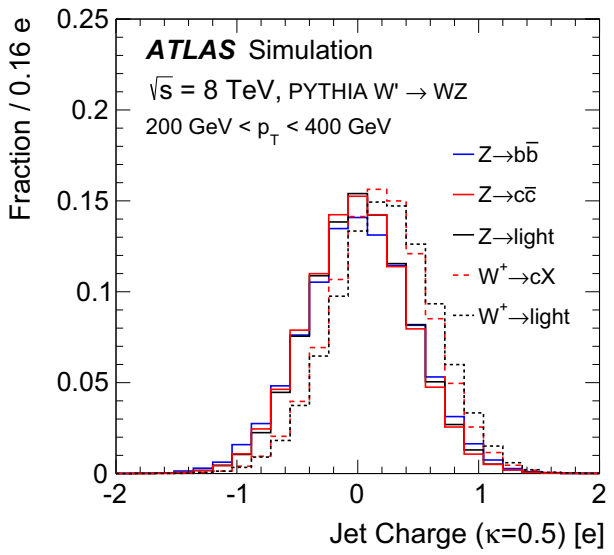

(b)

Fig. 5 a The jet mass $p(M \mid \mathcal{F}, V)$ and $\mathbf{b}$ jet charge $p(Q \mid \mathcal{F}, V)$ templates conditioned on the flavour $\mathcal{F}$ of the boson $V$ decay for jets with 200 $\mathrm{GeV}<p_{\mathrm{T}}<400 \mathrm{GeV}$. The solid lines are for $Z$ boson decays and the dashed lines are for $W$ boson decays

matched to the large-radius jets. These cases are all predicted to originate from light-quark decays of the $W$ and $Z$ bosons.

\section{Tagger performance}

The optimal multivariate tagger combining jet mass, jet charge, and the MV1 of matched small-radius jets is constructed from a three-dimensional (3D) likelihood ratio. For $N$ bins each of jet mass and jet charge, as well as 30 combined MV1 bins, the 3D likelihood ratio would have $30 \times N^{2}$ total bins. Populating all of these bins with sufficient $\mathrm{MC}$ events to produce templates for the likelihood ratio requires an unreasonable amount of computing resources, especially for the high-efficiency bins of combined MV1. Estimating the 3D likelihood as the product of the 1D marginal distributions, where all variables but the one under consideration are integrated out, is a poor approximation for jet mass and combined MV1 due to the correlation induced by the presence of semileptonic $b$-decays, which shift the jet mass to lower values due to the presence of unmeasured neutrinos. ${ }^{6}$ It is still possible to use a simple product by noting that all three tagger inputs are independent when the flavour of the decaying boson has been determined. Thus, for each possible boson decay channel, templates are built for the jet mass, the jet charge, and the efficiency-binned MV1 distributions. For a particular decay flavour, the joint distribution

\footnotetext{
$\overline{6}$ The muons from semileptonic decays are added back to the jet using a four-momentum sum. Muons are measured by the combination of a dedicated muon spectrometer with its own toroidal magnetic field outside the calorimeters, and the inner detector. Adding back the muon has a negligible impact on the inclusive mass distribution due to the semileptonic branching ratios and lepton identification requirements. For details about the muon reconstruction and selection, see Sect. 7 (the only difference here is that the isolation is not applied).
}

is then the product of the individual distributions. Summing over all hadronic decay channels then gives the full distribution. To ease notation, the efficiency-binned MV1 is denoted $B=\left(B_{\text {lead }}, B_{\text {sub-lead }}\right)$. The distribution for $B_{\text {lead }}\left(B_{\text {sub-lead }}\right)$ is shown in the left (right) plot in Fig. 4. Symbolically, for decay flavour channel $\mathcal{F}$, mass $M$, charge $Q$, and efficiency-binned MV1 $B$, the likelihood is given by:

$$
\begin{aligned}
p( & M, Q, B \mid V) \\
& =\sum_{\mathcal{F}} \operatorname{Pr}(\mathcal{F} \mid V) p(M \mid \mathcal{F}, V) p(Q \mid \mathcal{F}, V) \operatorname{Pr}(B \mid \mathcal{F}, V),
\end{aligned}
$$

where $^{7} V \in\{W, Z\}$ and the sum is over $\mathcal{F}=b b, c c, c s, c d$ and light-quark pairs. The distribution of $B$ is well approximated as the product of the distributions for $B_{\text {lead }}$ and $B_{\text {sub-lead }}$ when the flavours of the leading and sub-leading jets are known. This is exploited for hadronically decaying $W$ bosons and for the light-quark flavour decays of $Z$ bosons to construct templates for $B$ that have a sufficient number of simulated events for large values of $B$, i.e. $\operatorname{Pr}(B \mid \mathcal{F}, V)=\operatorname{Pr}\left(B_{\text {lead }} \mid \mathcal{F}, V\right) \operatorname{Pr}\left(B_{\text {sub-lead }} \mid \mathcal{F}, V\right)$. The unitnormalized templates for $B$ are shown in Fig. 4 and the unitnormalized templates $p(M \mid \mathcal{F}, V)$ and $p(Q \mid \mathcal{F}, V)$ are shown in Fig. 5. For a given boson type, the jet-charge template is nearly independent of the flavour. However, there is a dependence of the jet mass on the (heavy) flavour of the boson decay products.

The likelihood function is constructed by taking the ratio of the probability distribution functions $p(M, Q, B \mid V)$, for $V \in\{W, Z\}$, determined from the templates in Eq. (3). Every bin $i$ of the 3D histogram that approximates $p(M, Q, B \mid V)$ is assigned a pair of numbers $\left(i, s_{i} / b_{i}\right)$ where $s_{i}$ is the overall

\footnotetext{
7 The symbol $p$ denotes a probability density whereas Pr denotes a discrete probability distribution.
} 
fraction of the signal ( $Z$ or $W$ ) in bin $i$ and $b_{i}$ is the fraction of the overall background (the other boson flavour) in bin $i$. Bins are then sorted from largest to smallest $s_{i} / b_{i}$, with $f(i)$ defining a map from the old bin index to the new, sorted one. There are then two 1D histograms: for the signal, bin $j$ has bin content $s_{f^{-1}(j)}$ and for the background, bin $j$ has bin content $b_{f^{-1}(j)}$. The optimal tagging procedure is then to set a threshold on the new 1D histograms. The full likelihood ratio of the combined tagger is shown in Fig. 6 where the thresholds required for 90,50 , and $10 \% \mathrm{Z}$-boson tagging efficiency are marked with shaded regions.

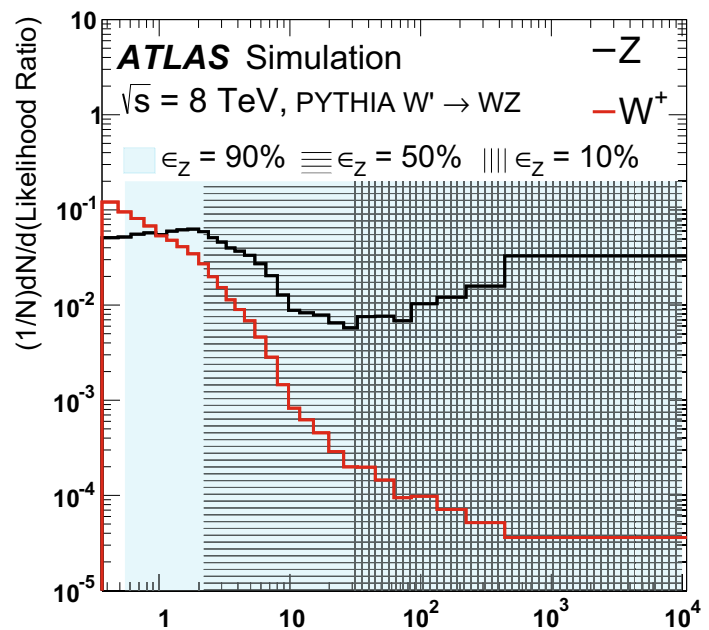

Full Likelihood Ratio (Jet Mass+Jet Charge+b-tagging)

Fig. 6 The full likelihood ratio for the tagger formed from jet mass, jet charge, and a small-radius jet $b$-tagging discriminant. The black histogram shows the likelihood ratio for $Z$ bosons and the red histogram is the likelihood ratio for $W^{+}$bosons. The shaded areas show the region of the likelihood ratio corresponding to 90,50 , and $10 \%$ working points of the $Z$-boson tagging efficiency

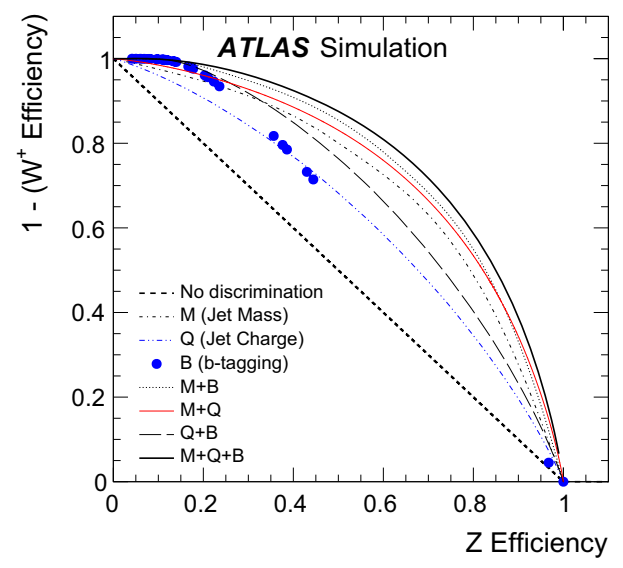

(a)

Fig. 7 The tradeoff between $Z$ efficiency and a $1-\left(W^{+}\right.$efficiency) $\mathbf{b}$ or $1 /\left(W^{+}\right.$efficiency) on a linear scale and $\mathbf{b}$ a logarithmic scale. Each curve is constructed by placing thresholds on the likelihood constructed
Curves displaying the tagging performance for all possible subsets of $\{M, Q, B\}$ are shown in Fig. 7. There are 30 possible values for $B$, which are therefore represented by discrete points. The jet mass is the best performing single variable for medium to high $Z$-boson efficiencies, with visible improvement for $M+B$ and $M+Q$. There is a significant gain from combining all three variables for $Z$-boson tagging efficiency above about $20 \%$. Below $20 \%$, the combined tagger is dominated by $B$ where the $Z \rightarrow b \bar{b}$ branching fraction no longer limits $Z$-boson tagging efficiency. For $Z$-boson efficiencies of about $50 \%$, one can achieve $W^{+}$rejection factors $\left(1 / \epsilon_{W^{+}}\right)$of 3.3 by using $Q$ or $B$ alone and about 5.0 using mass alone. For $Z$ efficiencies of $\epsilon_{Z}=90,50$, and $10 \%, W^{+}$ rejection factors of $1.7,8.3$, and 1000 , respectively, can be achieved with the combined tagger. Although most applications of boson-type tagging will target $Z$ bosons as the signal while rejecting $W$ bosons as background, the likelihood constructed in Fig. 6 can also be used to optimally distinguish $W^{+}$bosons from $Z$ bosons. The corresponding performance curves are shown in Fig. 8. The locations of the $b$-tagging points are all now shifted to high efficiency with respect to Fig. 7 because, for $W^{+}$tagging, one wants to operate in the high-efficiency $b$-tagging bins (whereas the opposite is optimal for $Z$ tagging). At an efficiency of $\epsilon_{W^{+}}=50 \%$, a $Z$-boson rejection factor of $1 / \epsilon_{Z} \approx 6.7$ can be achieved.

\section{Systematic uncertainties}

The performance curves in Fig. 7 are based on the nominal modelling parameters of the ATLAS simulation. Additional studies show how the curves change due to the systematic uncertainties on the inputs to the likelihood func-

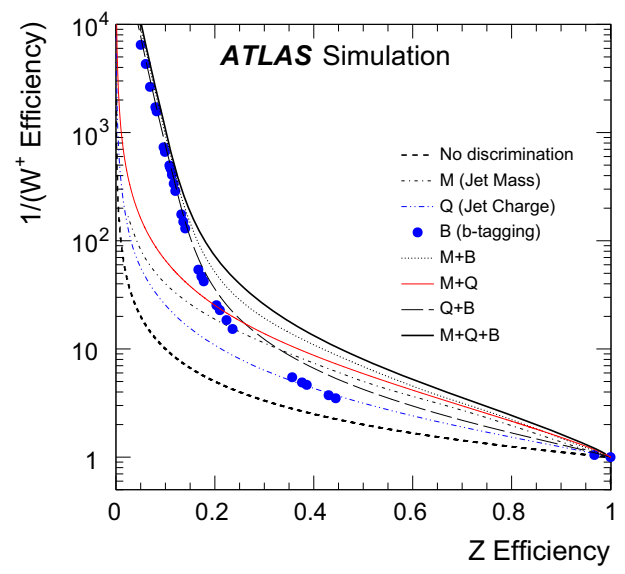

(b)

from the inputs indicated in the legend. Since the $b$-tagging discriminant is binned in efficiency, there are only discrete operating points for the tagger built only from $B$ 


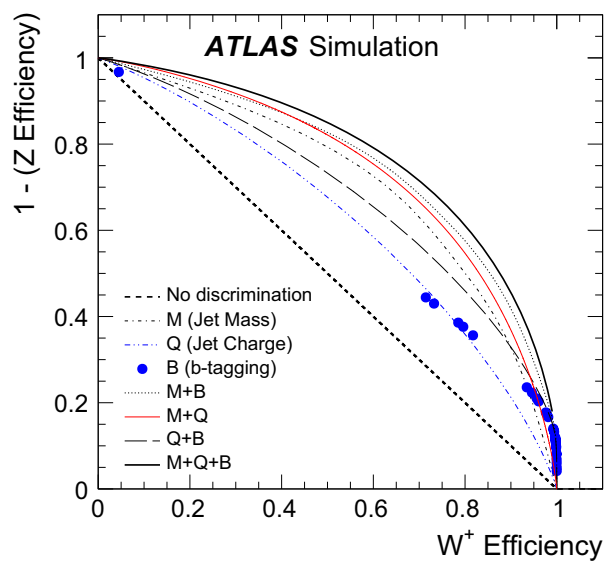

(a)

Fig. 8 The tradeoff between $W^{+}$efficiency and a $1-$ ( $Z$ efficiency) or b $1 /$ ( $Z$ efficiency) on a a linear scale and $\mathbf{b}$ a logarithmic scale. Each curve is constructed by placing thresholds on the likelihood constructed
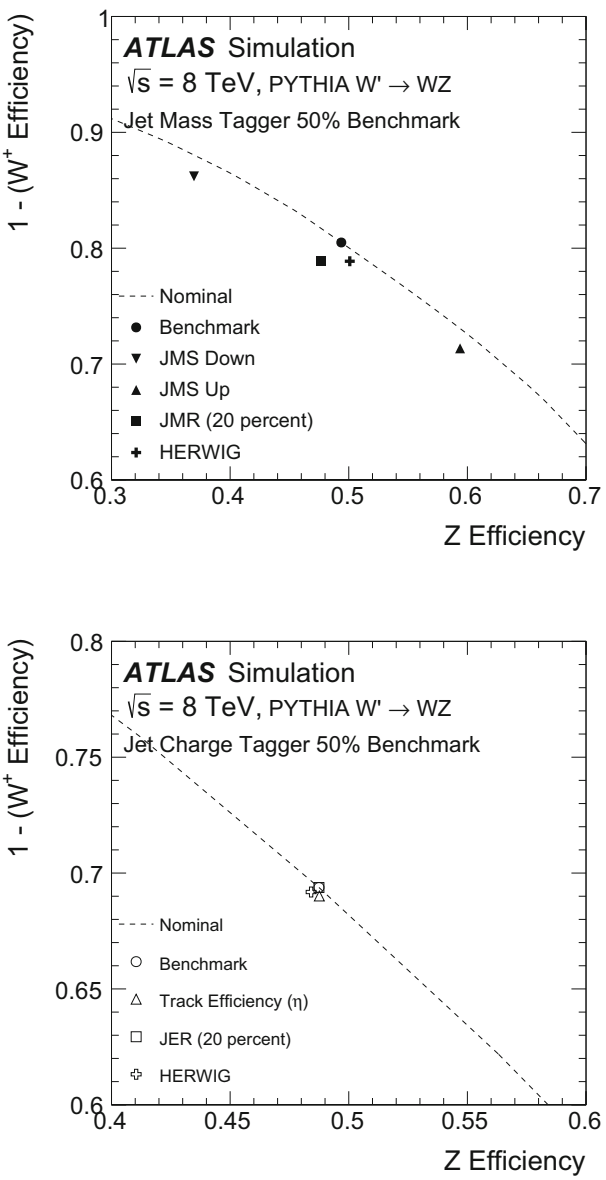

Fig. 9 The impact of selected systematic uncertainties on benchmark working points of the boson-type tagger. a A jet-mass-only tagger, for $50 \%$ (left) and $90 \% \mathrm{Z}$ efficiency benchmarks. b A jet-charge-only tagger, for $50 \%$ (left) and $90 \% \mathrm{Z}$ efficiency benchmarks. The point marked

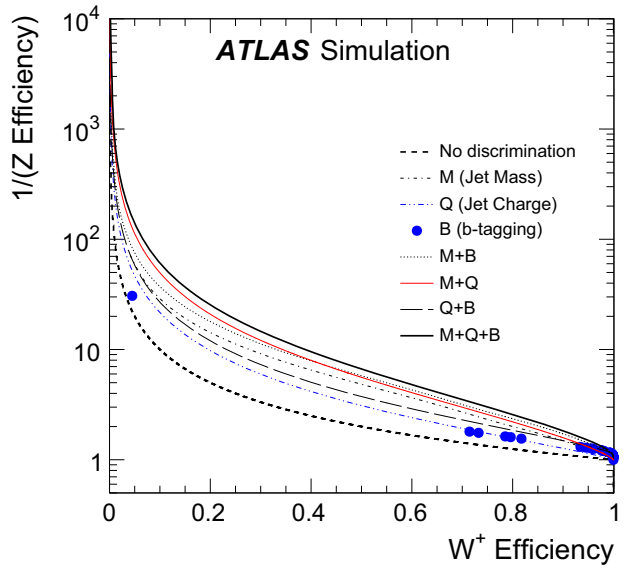

(b)

from the inputs indicated in the legend. Since the $b$-tagging discriminant is binned in efficiency, there are only discrete operating points for the tagger built only from $B$

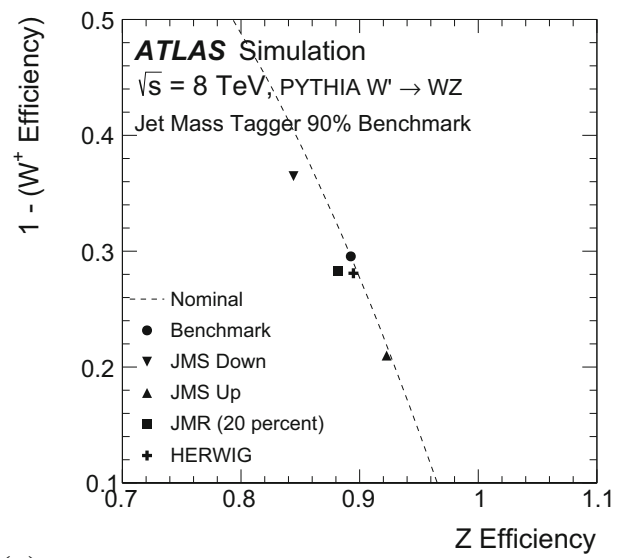

(a)

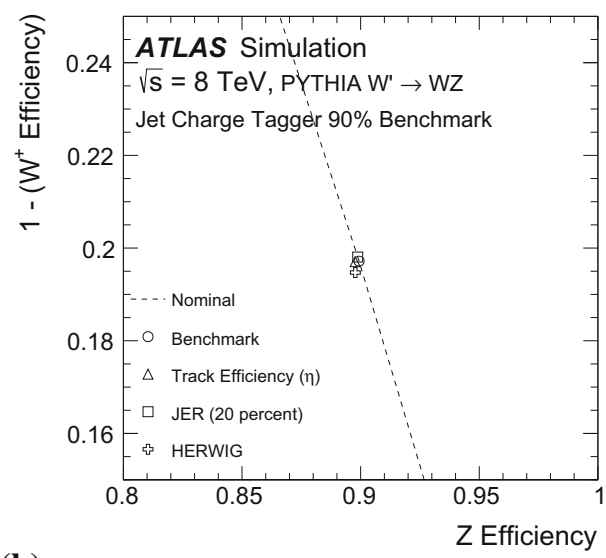

(b)

HERWIG uses the alternative shower and hadronization model for the simulation, with the likelihood template from PYTHIA. See the text for an explanation of the notation in the legend 
tion. Sources of experimental uncertainty include the calibrations of the large- and small-radius jet four-momenta, the $b$-tagging (which incorporates e.g. impact parameter modelling), and the modelling of track reconstruction.

The uncertainty on the scale of the large-radius jet mass calibration is estimated using the double ratio in data and MC simulation of calorimeter jet mass to track jet mass [45]. Tracks associated with a jet are well measured and provide an independent observable correlated with the jet energy. Uncertainties on the jet-mass resolution can have a non-negligible impact on the performance of the tagger. The jet-mass resolution uncertainty is determined from the difference in the widths of the boosted $W$ boson jet-mass peak in semileptonic $t \bar{t}$ simulated and measured data events [45] and also from varying the simulation according to its systematic uncertainties [46]. The resolution is about $5 \mathrm{GeV}$ in the Gaussian core of the mass spectrum and its uncertainty is about $20 \%$. The impact of the jet-mass scale and resolution uncertainties on the boson-type tagger built using only the jet mass is shown in Fig. 9 for two nominal working points of 50 and $90 \%$ $Z$-boson tagging efficiency. Both the likelihood map $f$ from Sect. 5 and the threshold value are fixed. Inputs to the tagger are shifted by their uncertainties and the 1D histograms described above are re-populated. The efficiencies for $W$ and $Z$ bosons are recomputed and shown as markers in Fig. 9a. Coherent shifts of the jet masses (JMS) for $W$ and $Z$ bosons result in movement along the nominal performance curve corresponding to $\pm 10 \%$ changes in the efficiency. However, there are also shifts away from the nominal curve because the optimal jet-mass cut is not a simple threshold. Variation of the jet-mass resolution (JMR) preserves the scale and so the movement is nearly perpendicular to the original performance curve, at the $\lesssim 5 \%$ level, because of the increased overlap in the $Z$ and $W$ mass distributions. ${ }^{8}$ Shifts along the nominal curve optimally use the input variables (albeit at different efficiencies), while shifts away from the nominal curve are a degradation in the performance. The impact of the fragmentation is estimated by using input variables from HERWIG but with the likelihood map from PYTHIA. PYTHIA and HERWIG have similar $W / Z$ efficiencies at both the 50 and $90 \%$ benchmark points.

The systematic uncertainty on the efficiency of the tracking reconstruction is estimated by removing tracks associated with jets using an $\eta$-dependent probability [47]. The probability in the region $2.3<|\eta|<2.5$ is $7 \%$; it is $4 \%$ for $1.9<|\eta|<2.3,3 \%$ for $1.3<|\eta|<1.9$, and $2 \%$ for $0<|\eta|<1.3$. These probabilities are known to be conservative in the most central $\eta$ bins. There is also an uncertainty on the modelling of track merging for high- $p_{\mathrm{T}}$ jets, but the

\footnotetext{
$\overline{8}$ Although such shifts retain optimal use of the tagger (highest rejection for a fixed efficiency), they can degrade the quality of e.g. a cross-section measurement.
}

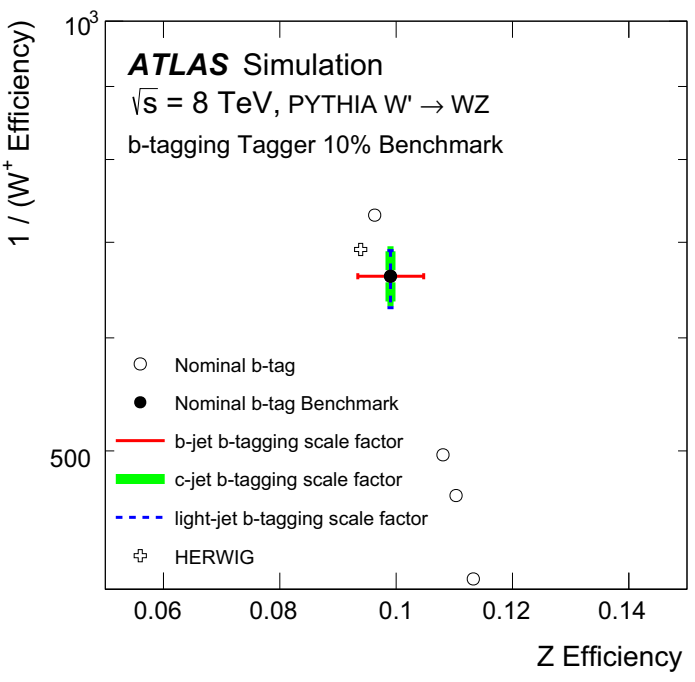

Fig. 10 The impact of selected systematic uncertainties on benchmark working points of a $b$-tagging-only tagger at a $10 \% \mathrm{Z}$ efficiency benchmark. The $b$-tagging discriminant is binned, so there are only discrete operating points. The point marked HERWIG uses the alternative shower and hadronization model for the simulation, with the likelihood template from PYTHIA. The $b$-tagging scale factor uncertainties are determined separately for $b-, c-$, and light-quark jets. Variations are added in quadrature for each 'truth' jet flavour. There is no contribution from the $b$-jet scale factor uncertainties on the $W$ rejection because there are no 'truth' $b$-jets. Conversely, the $c$ - and light-jet scale factor uncertainties do not impact the $Z$ bosons because at this low efficiency, all the selected $Z$ bosons decay into $b \bar{b}$

loss is expected to be negligible for jets with $p_{\mathrm{T}}<400 \mathrm{GeV}$. Differences in the modelling of fragmentation can affect the expected performance for all the input variables, especially for the track-dependent observables. The impact of various uncertainties on the boson-type tagger built using only the jet charge is shown in Fig. 9b. Since $W$ and $Z$ boson decays produce on average many tracks (see Sect. 7), removing a small number of them does not have a big impact on the jet-charge tagger as a result of the $p_{\mathrm{T}}$-weighting in the jet charge sum.

The efficiency to $b$-tag jets of various flavours $(b, c$, and light) is measured in data using $t \bar{t}$ events [43], jets with identified charm hadrons, and multijet events [44]. The differences between data and MC simulation are typically a few percent and are applied as independent correction factors on a per-jet basis. The uncertainties on these scale factor measurements are used as estimates of the systematic uncertainty on the $b$-tagging. The sources of uncertainty are decomposed into many uncorrelated components ( 24 for $b$-jets, 16 for $c$-jets, and 48 for light-flavour jets) and the impact on the rejection is added in quadrature for a fixed value of $\epsilon_{\text {signal. }}$. The $b$-tagging of matched small-radius jets is also affected by uncertainties on the jet-energy scale and resolution. These quantities are varied within their uncertainties and if the shifted jet has $p_{\mathrm{T}}<25 \mathrm{GeV}$, its MV1 value is not considered. The impact of various uncertainties on the boson-type tagger built using 


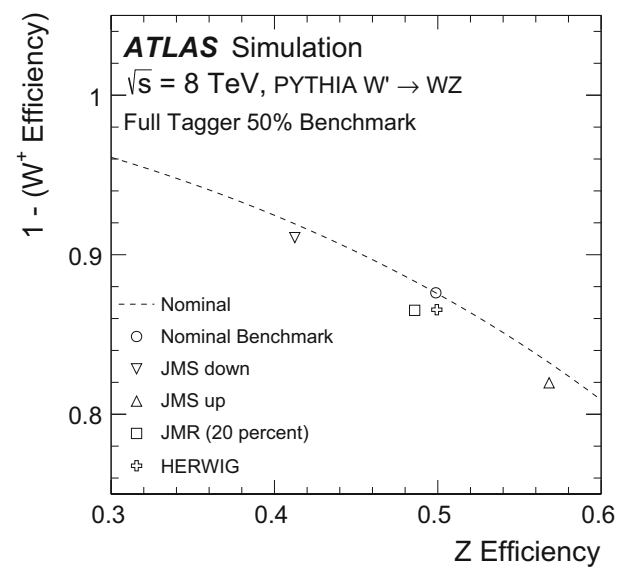

(a)

Fig. 11 The impact of uncertainties on the jet-mass scale and resolution for $50 \%$ (a) and $90 \%$ (b) $Z$ efficiency working points of the full boson-type tagger. The point marked HERWIG uses the alternative

only the $b$-tagging discriminant for a $10 \%$ nominal $Z$ efficiency is shown in Fig. 10. At this efficiency, the full bosontype tagger is dominated by the $b$-tagging inputs, as seen in Fig. 7. The scale factor uncertainty for $b$-jets has no impact on the $W$ efficiency (no real $b$-jets), but there is approximately a $10 \%$ uncertainty on the $Z$ efficiency. The uncertainties on the jet-energy scale for small-radius jets are relevant only because of the $25 \mathrm{GeV} p_{\mathrm{T}}$ threshold. Since all of the largeradius jets are required to have $p_{\mathrm{T}}>200 \mathrm{GeV}$, the threshold is relevant only in the rare case that one of the $W$ daughters is nearly anti-parallel in the $W$ rest frame to the direction of the $W$ boost vector.

The impact of the uncertainties on the jet-mass scale and resolution on the boson-type tagger built using all of the inputs (jet mass, jet charge, and $b$-tagging) is shown in Fig. 11a. At very low $Z$-boson tagging efficiency, the tagger is dominated by $b$-tagging, so Fig. 10 is a good representation of the uncertainty on the full tagger's performance. For higher efficiencies, the tagger is dominated by the jet mass, although the jet charge and $b$-tagging discriminant significantly improve the performance. The uncertainty on the full tagger's performance at the 50 and $90 \% \mathrm{Z}$-boson tagging efficiency benchmark points is due mostly to the uncertainty on the jet mass, which is why these uncertainties are shown in Fig. 11.

\section{Validation of tagging variables using data}

The tagger cannot be fully tested with data because it is not possible to isolate a pure sample of hadronically decaying $Z$ bosons in $p p$ collisions. However, the modelling of the variables used to design the tagger can be studied with a relatively pure and copious sample of hadronically decaying

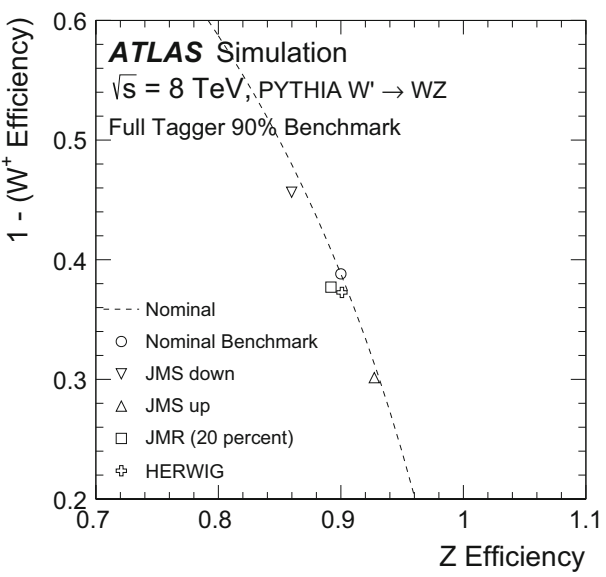

(b)

shower and hadronization model for the simulation, with the likelihood template from PYTHIA

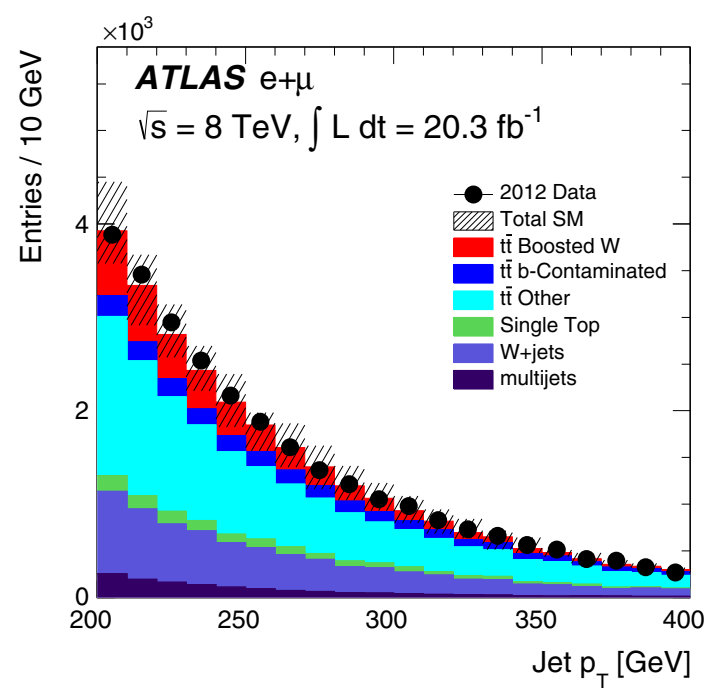

Fig. 12 The $p_{\mathrm{T}}$ distribution of the selected large-radius jets. The uncertainty band includes all the experimental uncertainties on the jet $p_{\mathrm{T}}$ and jet mass described in Sect. 6

$W$ bosons in $t \bar{t}$ events which can be tagged by the leptonic decay of the other $W$ boson in the event (semileptonic $t \bar{t}$ events). Single-lepton triggers are used to reject most of the events from QCD multijet background processes. Candidate reconstructed $t \bar{t}$ events are chosen by requiring an electron or a muon with $p_{\mathrm{T}}>25 \mathrm{GeV}$ and $|\eta|<2.5$, as well as a missing transverse momentum $E_{\mathrm{T}}^{\text {miss }}>20 \mathrm{GeV}$. The electrons and muons are required to satisfy a series of quality criteria, including isolation. ${ }^{9}$ Events are rejected if there is

\footnotetext{
${ }^{9}$ Leptons are considered isolated if they are well separated from jets $(\Delta R>0.4)$ and the track/calorimeter energy within a small cone, centred on the lepton direction but excluding the lepton itself, is below a fixed relative value.
} 


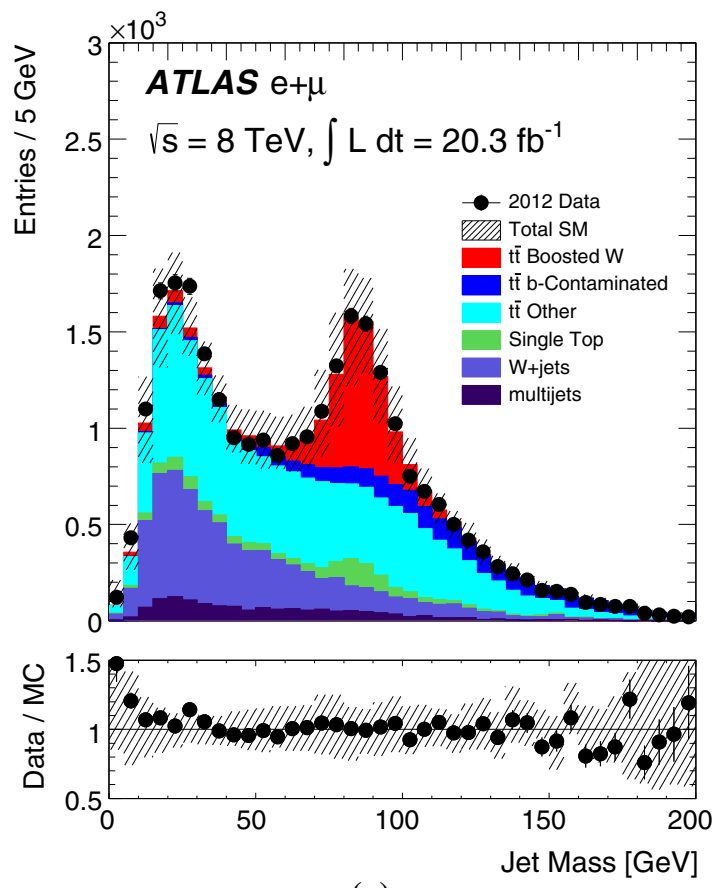

(a)

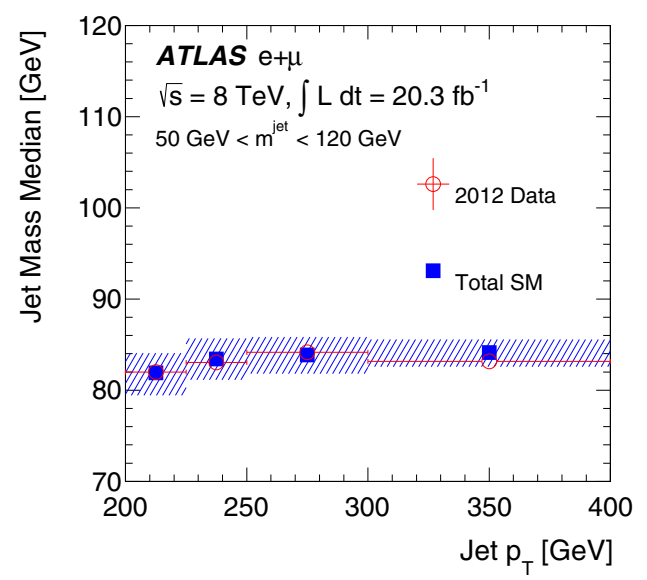

(b)

Fig. 13 a The jet-mass distribution of the selected jets in semi-leptonic $t \bar{t}$ events. $\mathbf{b}$ The median of the mass distribution as a function of the jet $p_{\mathrm{T}}$ for events with the selected jet in the range $50 \mathrm{GeV}<m^{\text {jet }}<$ $120 \mathrm{GeV}$. This includes the contributions from events which are not classified as Boosted $W$. c For the same events as in $\mathbf{b}$, the inter-quantile range as a measure of spread. The quantiles are centred at the median.

not exactly one electron or muon. In addition, the sum of the $E_{\mathrm{T}}^{\text {miss }}$ and the transverse mass ${ }^{10}$ of the $W$ boson, reconstructed from the lepton and $E_{\mathrm{T}}^{\text {miss }}$, is required to be greater than $60 \mathrm{GeV}$. Events must have at least one $b$-tagged jet (at the $70 \%$ efficiency working point) and have at least one largeradius trimmed jet with $p_{\mathrm{T}}>200 \mathrm{GeV}$ and $|\eta|<2$. Further-

\footnotetext{
10 The transverse mass, $m_{\mathrm{T}}$, is defined as $m_{\mathrm{T}}^{2}=2 p_{\mathrm{T}}^{\text {lep }} E_{\mathrm{T}}^{\text {miss }}(1-$ $\cos (\Delta \phi))$, where $\Delta \phi$ is the azimuthal angle between the lepton and the direction of the missing transverse momentum.
}

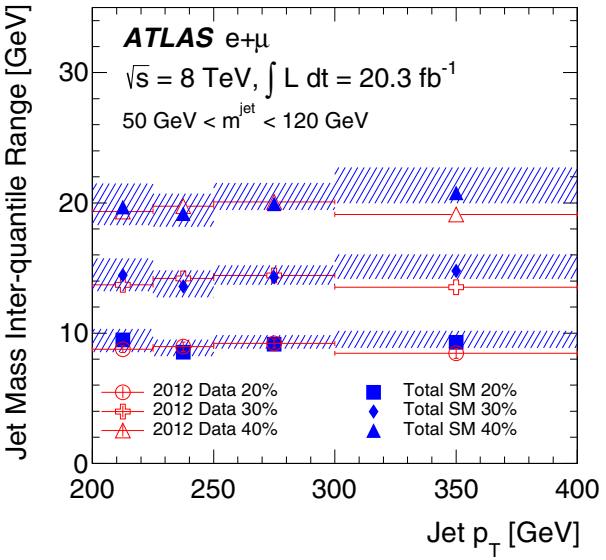

(c)

The uncertainty band includes all the experimental uncertainties on the jet $p_{\mathrm{T}}$ and jet mass described in Sect. 6. The inter-quantile range of size $0 \%<X<50 \%$ is defined as the difference between the $50 \%+X \%$ quantile and the $50 \%-X \%$ quantile. Statistical uncertainty bars are included on the data points but are smaller than the markers in many bins

more, there must be a small-radius jet with $p_{\mathrm{T}}>25 \mathrm{GeV}$, and $\Delta R<1.5$ to the selected lepton (targeting the decay chain $t \rightarrow b W(\rightarrow \ell v))$. The other $W$ boson candidate is selected as the leading large-radius trimmed jet with $\Delta R>1.5$ from the small-radius jet that is matched to the lepton. The $W+$ jets and multijet backgrounds are estimated from the data using the charge asymmetry and matrix methods, respectively [48]. The other backgrounds are estimated directly from MC simulation. Although the resulting event selection is expected 


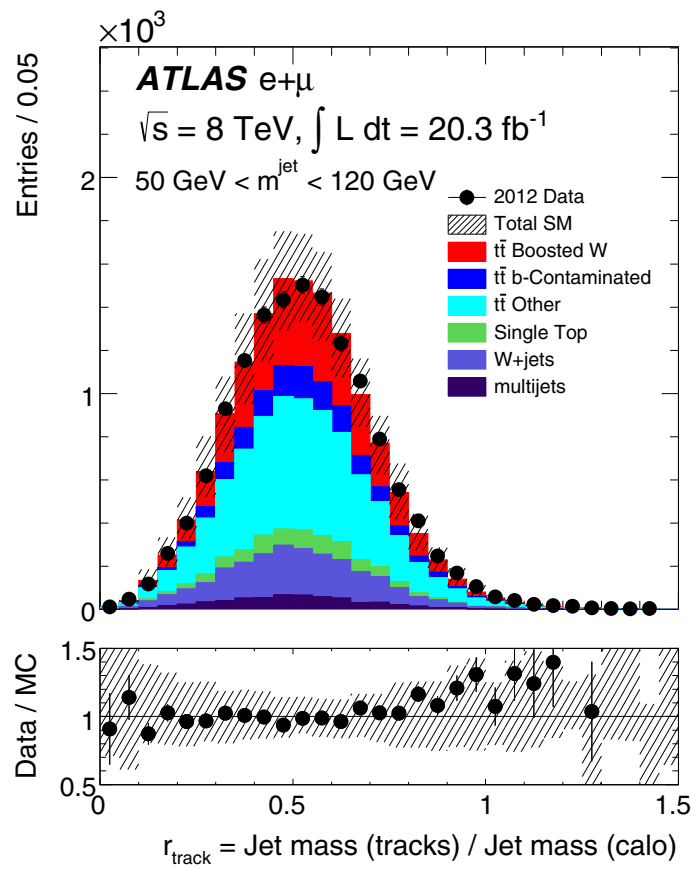

(a)

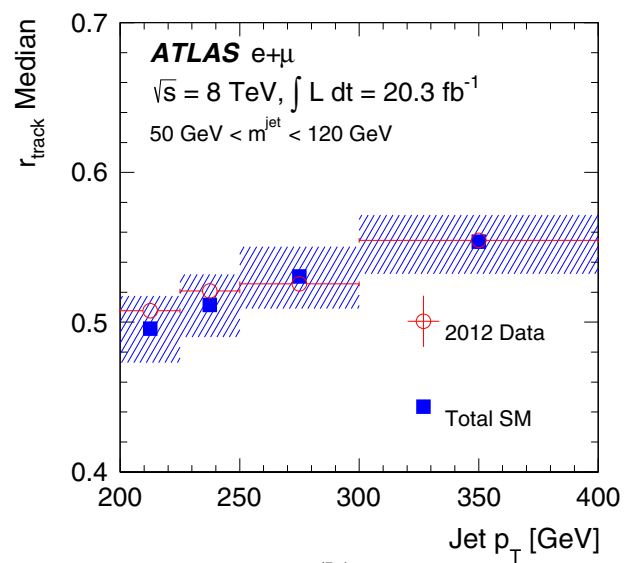

(b)

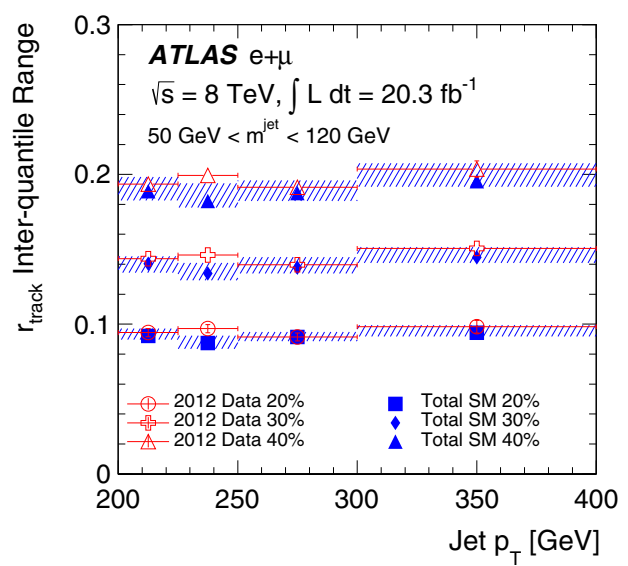

(c)

Fig. 14 a The distribution of $r_{\text {track }}$ in the data for semi-leptonic $t \bar{t}$ events with the selected jet in the range $50 \mathrm{GeV}<m^{\text {jet }}<120 \mathrm{GeV}$. b The median of the $r_{\text {track }}$ distribution as a function of the jet $p_{\mathrm{T}}$. This includes the contributions from events that are not classified as Boosted $W$. c The inter-quantile range as a measure of the width. The quantiles are centred at the median. The uncertainty band includes all the exper-

to have a high $t \bar{t}$ purity (about $75 \%$ ), the events cannot be compared directly to the isolated $W$ bosons from the simulated $W^{\prime}$ boson decays. This is because there are several effects that make the typical large-radius jet in semileptonic $t \bar{t}$ events different from isolated $W$ and $Z$ boson jets in $W^{\prime}$ boson events: ${ }^{11}$

\footnotetext{
11 When controlling for all differences, the distributions for isolated $W$ bosons from $t \bar{t}$ and from $W^{\prime}$ are nearly identical.
}

imental uncertainties on the jet $p_{\mathrm{T}}$ and jet mass described in Sect. 6 . The inter-quantile range of size $0 \%<X<50 \%$ is defined as the difference between the $50 \%+X \%$ quantile and the $50 \%-X \%$ quantile. Statistical uncertainty bars are included on the data points but are smaller than the markers in many bins

1. The event selection is based on the reconstructed jet $p_{\mathrm{T}}$ (earlier sections used $p_{\mathrm{T}}^{V}$ ), so even if $p_{\mathrm{T}}^{\text {jet }} \gtrsim 200 \mathrm{GeV}$ for an $R=1.0$ jet, the true hadronically decaying $W$ boson in the event may have $p_{\mathrm{T}}^{W}<200 \mathrm{GeV}$ and thus the $W$ boson decay products might not be collimated within $\Delta R<1$.

2. There are more (close-by) jets in semileptonic $t \bar{t}$ events than in $W^{\prime}$ boson events. Jets not originating from the $W$ boson can form the leading large-radius jet, or the $b$-jet 


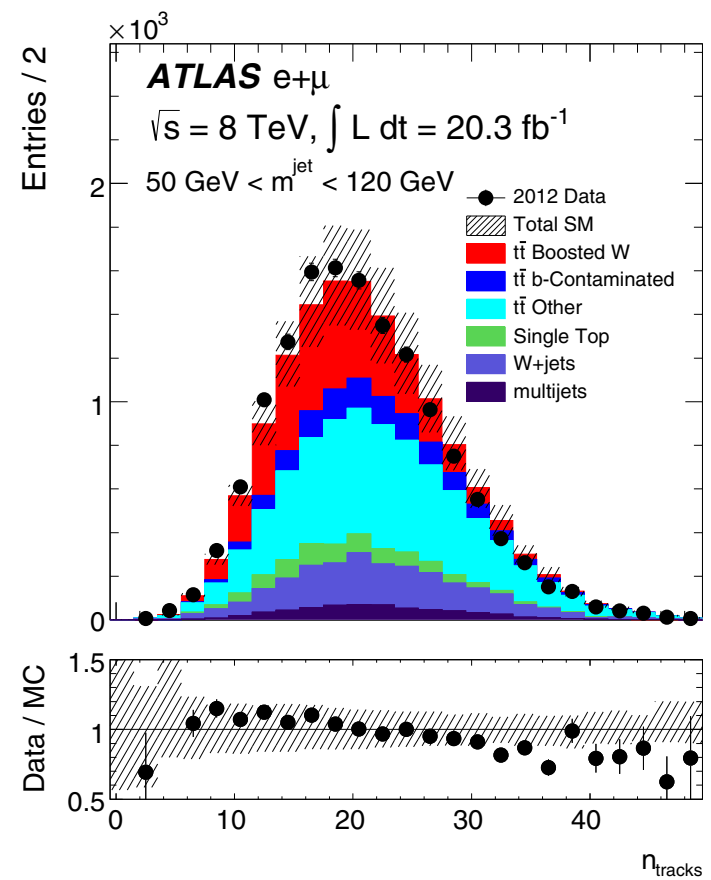

(a)

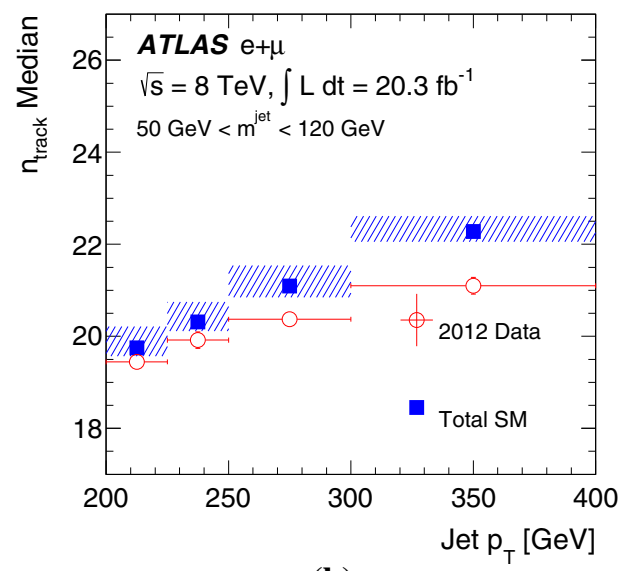

(b)

Fig. 15 a The distribution of the number of tracks associated with the selected large-radius jet in the semi-leptonic $t \bar{t}$ data for events with the selected jet in the range $50 \mathrm{GeV}<m^{\text {jet }}<120 \mathrm{GeV}$. b The median of the distribution of the number of tracks as a function of the jet $p_{\mathrm{T}}$. This includes the contributions from events that are not classified as Boosted $W$. c The inter-quantile range as a measure of the width. The

from the same top-quark as the hadronically decaying $W$ bosons can merge with the $W$ boson decay products to form a large-radius jet.

The variables $p_{\mathrm{T}}^{\text {jet }} / p_{\mathrm{T}}^{W}$ and $\Delta R(\mathrm{jet}, W)$, for the $W$ boson from the MC 'truth' record and the selected large-radius jet, are used to classify the various $t \bar{t}$ event sub-topologies. Events are labelled as having a Boosted $W$ if $\left|p_{\mathrm{T}}^{\text {jet }} / p_{\mathrm{T}}^{W}-1\right|<$

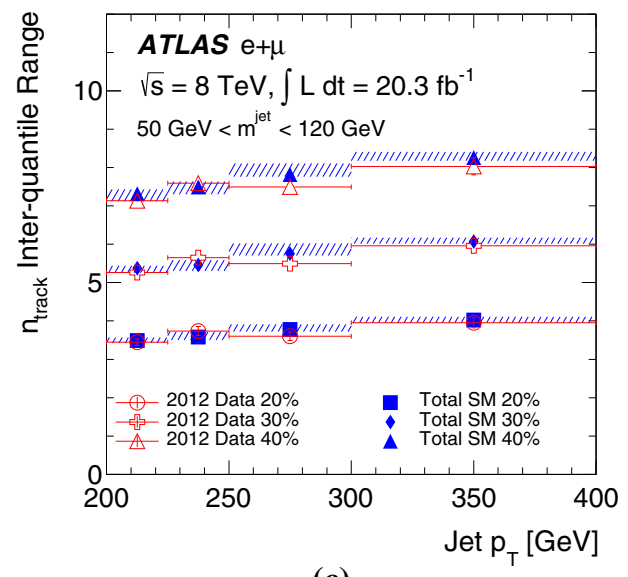

(c)

quantiles are centred at the median. The uncertainty band includes all the experimental uncertainties on the jet $p_{\mathrm{T}}$ and jet mass described in Sect. 6. The inter-quantile range of size $0 \%<X<50 \%$ is defined as the difference between the $50 \%+X \%$ quantile and the $50 \%-X \%$ quantile. Statistical uncertainty bars are included on the data points but are smaller than the markers in many bins

0.1 and $\Delta R($ jet, $W)<0.1$. If the $b$-quark from the top-quark decay has an angular distance $\Delta R<1.0$ from the selected large-radius jet, this jet is labelled as $b$-contaminated. All other $t \bar{t}$ events, including events where both $W$ bosons decay into leptons, are labelled as Other. The $p_{\mathrm{T}}$ spectrum of the jets from the classified events is shown in Fig. 12. In Fig. 12 and subsequent figures, systematic uncertainties on the simulation include the jet $p_{\mathrm{T}}$ and jet mass uncertainties described 


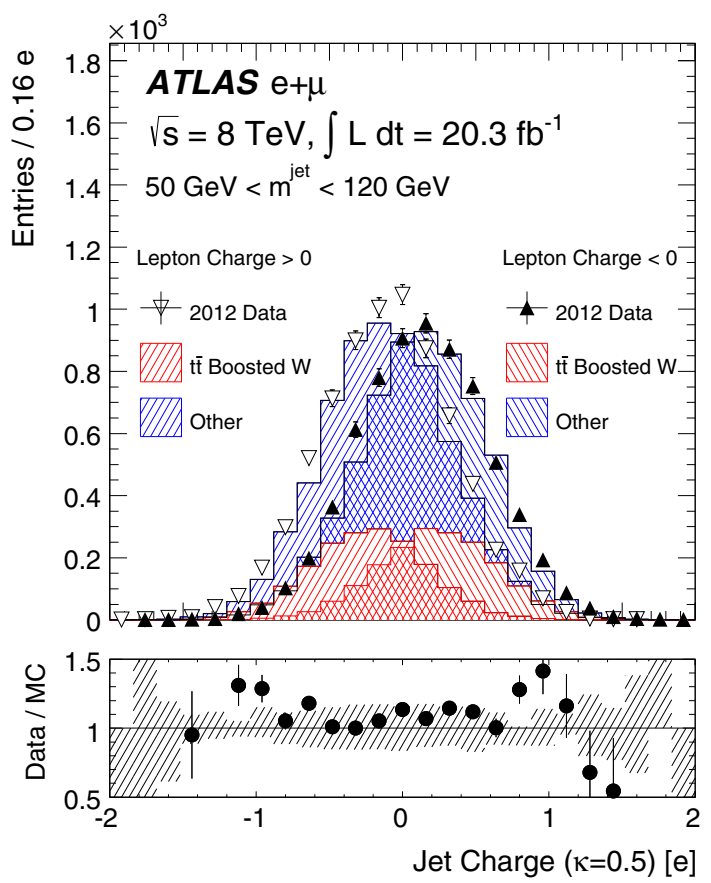

(a)

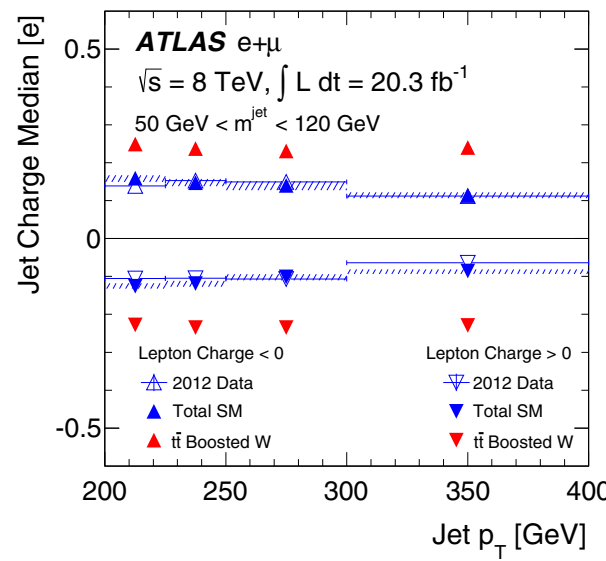

(b)

Fig. 16 a The distribution of the jet charge in the data for semi-leptonic $t \bar{t}$ events with the selected jet in the range $50 \mathrm{GeV}<m^{\text {jet }}<120 \mathrm{GeV}$. The ratio uses the positive lepton charge. $\mathbf{b}$ The median of the jet charge distribution as a function of the jet $p_{\mathrm{T}}$. This includes the contributions from events that are not classified as Boosted $W$ (except for the blue triangles, for which only the Boosted $W$ is included). c The inter-quartile

in Sect. 6, but exclude tracking uncertainties, which are subdominant. Events are vetoed if the selected large-radius jet has $p_{\mathrm{T}}>400 \mathrm{GeV}$ or if the $\Delta R$ between the selected largeradius jet and a tagged $b$-jet is less than 1.0. This suppresses the $b$-contaminated $t \bar{t}$ events. The effectiveness of the $t \bar{t}$ event classification is most easily seen from the jet mass distribution, shown in Fig. 13a. The mass of the boosted $W$ bosons from $t \bar{t}$ events is peaked around $m_{W}$, as is a small contribu-

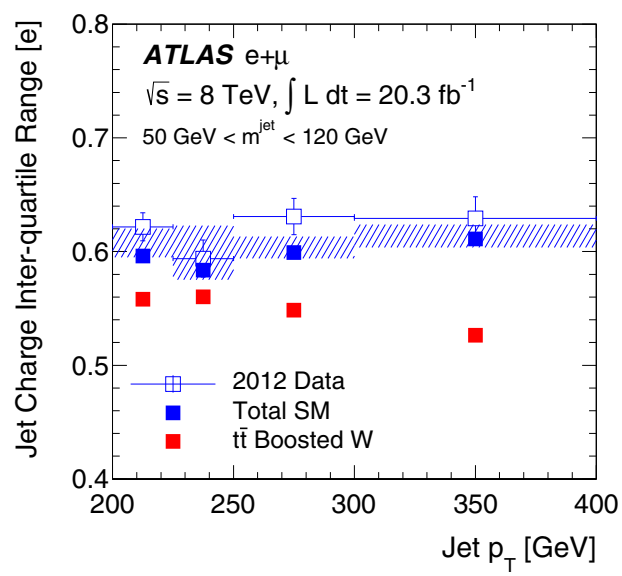

(c)

range as a measure of the width. The quantiles are centred at the median. The uncertainty band includes all the experimental uncertainties on the jet $p_{\mathrm{T}}$ and jet mass described in Sect. 6. The inter-quantile range is defined as the difference between the $75 \%$ quantile and the $25 \%$ quantile. Statistical uncertainty bars are included on the data points but are smaller than the markers in many bins

tion from the hadronically decaying $W$ bosons in single-top events in the $W t$ channel. There is no peak at $m_{t}$ in the $b$ contaminated spectrum because of the $b$-jet veto, but there is a small non-resonant contribution below the top-quark mass, due to events in which one $W$ daughter is matched with the $b$-jet. This is akin to the $b$-jet+lepton invariant mass used in other circumstances to measure top-quark properties and naturally has a scale around $150 \mathrm{GeV}$ [49]. The low-mass peak 


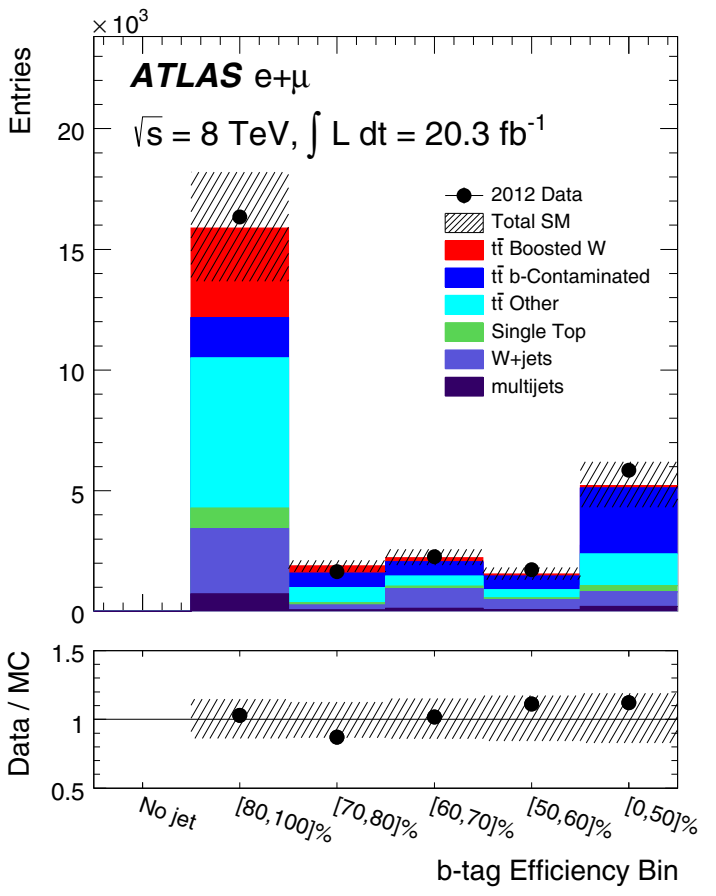

(a)

Fig. 17 The efficiency-binned MV1 distribution for the a leading and b sub-leading matched small-radius in semi-leptonic $t \bar{t}$ events. If there is no second small-radius jet with $p_{\mathrm{T}}>25 \mathrm{GeV}$ and $\Delta R<1$ to the selected large-radius jet axis, the event is put in the 'No jet' category in

in $W+$ jets and the 'other' $t \bar{t}$ events is due to the Sudakov peak from QCD jets, the location of which scales with $R \times p_{\mathrm{T}}$. The dependence on $p_{\mathrm{T}}$ of the $W$-peak position in Fig. 13a is shown in Fig. 13b. Events with the leading jet in a window around the $W$ mass, $50 \mathrm{GeV}<m^{\text {jet }}<120 \mathrm{GeV}$ are selected and the median of the mass distribution is plotted in Fig. $13 \mathrm{~b}$ as a function of the jet $p_{\mathrm{T}}$. The similar trend for the simulation and the data shows that the combination of the reconstructed jet-mass scale and 'truth' jet-mass scale is well modelled. To quantify the spread in the jet mass peak, various inter-quantile ranges are shown as a function of $p_{\mathrm{T}}$ in Fig. 13c. The inter-quantile range of size $0 \%<X<50 \%$ is defined as the difference between the $50 \%+X \%$ quantile and the $50 \%-X \%$ quantile, and is a measure of the spread in the distribution. The width of the boosted- $W$ mass peak is well modelled within the statistical precision of the 2012 data sample.

The modelling of boosted $W$ bosons can also be studied using the jet-mass scale measured from tracks. Defining the variable $r_{\text {track }}$ as the ratio of the jet mass determined from tracks to the jet mass determined from the calorimeter, the jet mass scale uncertainty is related to the difference from unity of the ratio of $\left\langle r_{\text {track }}\right\rangle$ in data to $\left\langle r_{\text {track }}\right\rangle$ in MC simulation. The mass scale uncertainty is calculated using the procedure described above, but with $r_{\text {track }}^{-1}$. If the jet consists only of

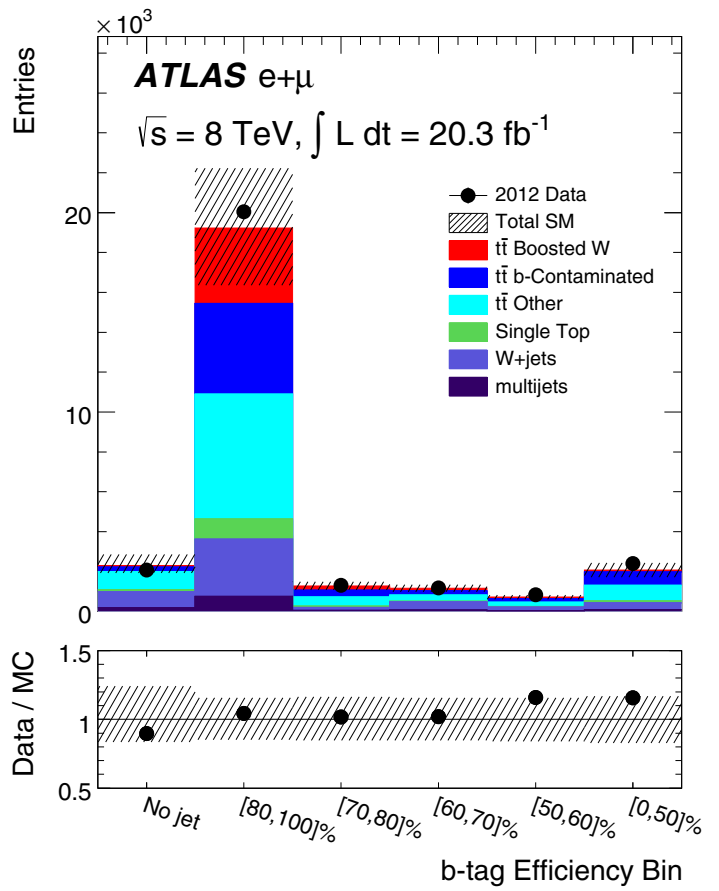

(b)

b. The uncertainty band includes all the experimental uncertainties on the jet $p_{\mathrm{T}}$ and jet mass and those related to the $b$-tagging described in Sect. 6. Statistical uncertainty bars are included on the data points but are smaller than the markers in many bins

pions, the natural scale for $r_{\text {track }}$ is $2 / 3$, although there are significant physics and detector effects that introduce a large spread of values. The distribution of $r_{\text {track }}$ in the $t \bar{t}$-enriched event sample with the same $p_{\mathrm{T}}$ and $b$-jet veto requirements as in Fig. 13 is shown in Fig. 14a. Unlike the raw jet-mass distribution, the $r_{\text {track }}$ distribution is similar for all of the sub-processes, as expected. The scale and spread of the $r_{\text {track }}$ distribution are quantified in Fig. 14b, c using the $p_{\mathrm{T}}$ dependence of the median and inter-quantile ranges. Previous studies have indicated that the track multiplicity, $n_{\text {track }}$, in quark and gluon jets is not well modelled, especially for gluon jets, where $n_{\text {track }}$ is lower in the data with respect to PYTHIA [50]. The distribution of the track multiplicity for large- $R$ jets in the $t \bar{t}$-enriched event sample is shown in Fig. 15. The boosted $W$ events are peaked at slightly lower values of the number of associated tracks compared to the quark/gluon jets from the other processes. The (charged) particle multiplicity increases for generic quark and gluon jets as a function of jet energy. However, the mass-scale of the jets produced from $W$ boson decays is set by $m_{W}$ so that in the absence of detector reconstruction effects, the track multiplicity distribution should be largely $p_{\mathrm{T}}$ independent. The $p_{\mathrm{T}}$ dependence of the track multiplicity is shown in Fig. 15b, c in the form of the median and the inter-quantile ranges. The median does increase because of the large non- $W$ component as well as the finite detector 


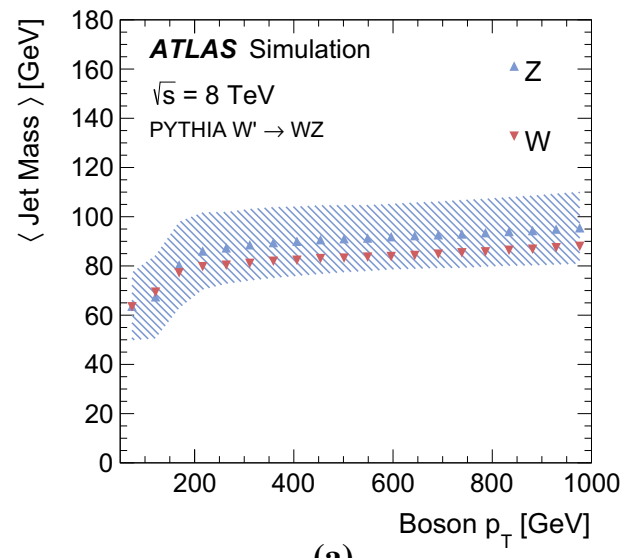

(a)

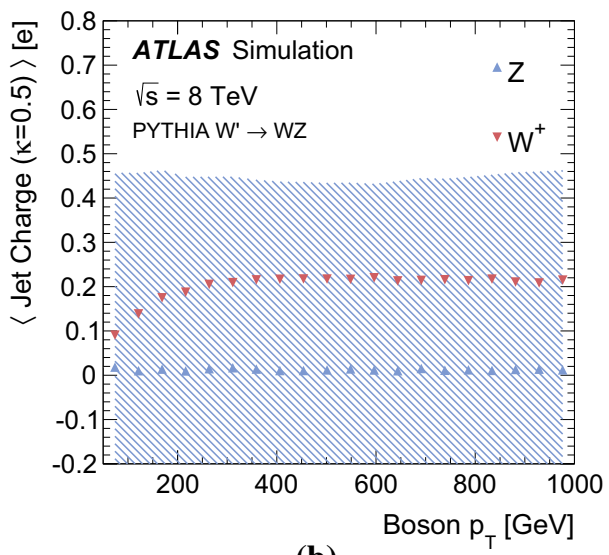

(b)

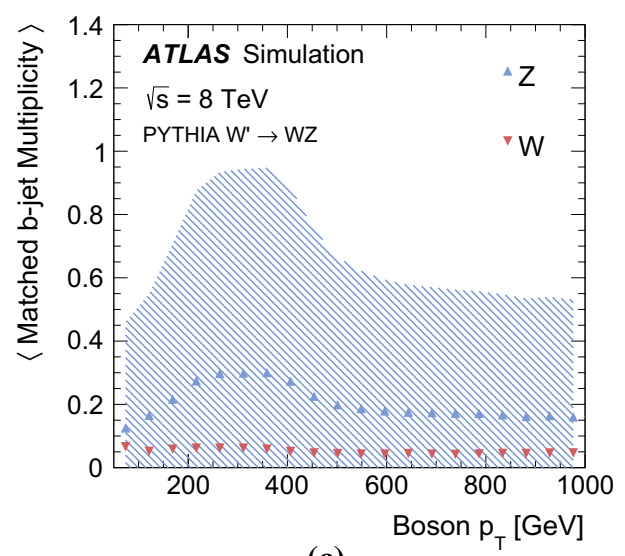

(c)

Fig. 18 The boson $p_{\mathrm{T}}$ dependence of the a jet mass, $\mathbf{b}$ jet charge, and $\mathbf{c}$ number of small-radius $b$-tagged jets matched to the large-radius jet

acceptance for charged particles from the boosted $W$ boson decay. The width is well modelled within the statistical precision of the data. However, there is disagreement for the median. Previous studies (including Rev. [50]) suggest that this is due to fragmentation modelling and not the modelling of the detector response.

The $p_{\mathrm{T}}$-weighted distribution of the track charges defines the jet charge, which is shown in Fig. 16a. The charge of the lepton from the leptonic $W$ boson decay determines the expected charge of the hadronically decaying $W$ boson candidate, allowing for a tag-and-probe study of the capability of charge tagging in hadronic $W$ boson decays [42]. The jet charge for boosted $W$ bosons for positively (negatively) charged leptons is clearly shifted to the left (right) of zero. There is also some separation between positive and negative $W$ boson decays when the selected large-radius jet does not satisfy the criteria for being a boosted $W$ boson. This is because the jet still contains some of the $W$ boson decay products, and the jet charge is correlated with the charge of the $W$ boson. The difference between the inclusive and boosted $W$-boson jets is clearer in the $p_{\mathrm{T}}$ dependence plot of the median jet charge shown in Fig. 16b. The medians of the distributions for boosted $W$ jets are nearly twice as far apart as the medians for inclusive jets. However, in both cases the spread is less than the width of the distribution, shown as the inter-quantile range (inter-quantile range with $X=25 \%$ ) in Fig. 16c. Even though there is some small disagreement for the median number of tracks, the $p_{\mathrm{T}}$-weighted sum defining the jet charge is reasonably well modelled.

The remaining input to the boson tagger is the $b$ tagging discriminant for the matched small-radius jets. The efficiency-binned MV1 distributions are shown in Fig. 17a, $\mathrm{b}$ with the same selection criteria as for the previous figures, except that the $b$-jet veto is removed. The contamination due to the $b$-jet from the top-quark decay complicates a direct study of the MV1 distribution for boosted $W$ jets; contamination from the $b$-quark decay products is seen clearly in the MV1 distribution at lower values of the efficiency. Most of the boosted $W$ jets are in the highest efficiency bin because they have no real $b$-hadron decay.

Overall, the simulation models all three input variables well. 


\section{Outlook}

The simulation studies of the boson-type tagger presented in Sect. 5 show that for bosons with $200 \mathrm{GeV}<p_{\mathrm{T}}<400 \mathrm{GeV}$, it is possible to achieve $Z$-boson efficiencies of $\epsilon_{Z}=90,50$, and $10 \%$ with $W^{+}$boson rejections of $1.7,8.3$ and 1000 , respectively. Putting this into context, with $R\left(\epsilon_{Z}\right)$ defined as the lowest possible $W$-boson tagging efficiency at a fixed $Z$-boson tagging efficiency:

- The $W Z / W W$ cross-section ratio is $\sim 20 \%$ [51]. At the $50 \%$ type-tagger working point, one can change the ratio of events to

$$
\begin{aligned}
\frac{50 \%}{R(50 \%)} \times \frac{\sigma(W Z)}{\sigma(W W)} & =\frac{50 \%}{12 \%} \times \frac{\sigma(W Z)}{\sigma(W W)} \\
& =\frac{50}{12} \times 20 \% \approx 83 \%
\end{aligned}
$$

with the possibility for a high-purity extraction of the $W Z$ cross section in the semileptonic channel $(\ell v q \bar{q})$.

- Diboson resonances are predicted by many models of physics beyond the Standard Model. The all-hadronic channel provides a significantly higher yield than the leptonic channels. At the $90 \%$ type-tagger working point, one can distinguish $Z Z$ from $W Z$ with a likelihood ratio of $0.9^{2} /(0.9 \times 0.6) \sim 1.5$. new resonance is discovered with $\sim 20$ events, this means that the difference between $Z Z$ and $W Z$ is distinguishable within a $2 \sigma$ statistical uncertainty of the data.

- At the $10 \%$ type-tagger working point, a leptophobic flavour-changing neutral current in $t \bar{t}$ production (with decays like in the $\mathrm{SM}$ ) with a branching ratio of $1 \%$ would have the same number of events as the $t \rightarrow b W$ decay: ${ }^{12}$

$$
\begin{aligned}
& \frac{10 \%}{R(10 \%)} \times \frac{\Gamma(t \rightarrow Z c)}{\Gamma(t \rightarrow W b)}=\frac{10 \%}{0.1 \%} \times \frac{\Gamma(t \rightarrow Z c)}{\Gamma(t \rightarrow W b)} \\
& \quad=100 \times 1 \%=100 \% .
\end{aligned}
$$

Only the range $200 \mathrm{GeV}<p_{\mathrm{T}}<400 \mathrm{GeV}$ was studied thus far due to the availability of $W$ bosons in the data. Figure 18 shows how the average and standard deviation of the jet mass, jet charge and multiplicity of the matched small-radius $b$-tagged jets distributions depend on jet $p_{\mathrm{T}}$ in simulation up to $1 \mathrm{TeV}$. As long as the jet $p_{\mathrm{T}}$ is high enough so that a single jet captures all of the boson decay products, the jet mass and jet charge distributions are predicted to be largely independent of $p_{\mathrm{T}}$. The information from $b$-tagging degrades around $400 \mathrm{GeV}$ as the two decay products from the boson become too close to resolve two separate jets.

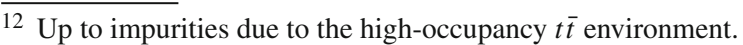

\section{Conclusions}

A tagger for distinguishing hadronically decaying boosted $Z$ bosons from $W$ bosons using the ATLAS detector has been presented. It will most likely be used after a boson tagger has rejected most QCD multijet events. Three discriminating variables are chosen which are sensitive to the differences in boson mass, charge, and branching ratios to specific quark flavours: large-radius jet mass, large-radius jet charge, and an associated small-radius jet $b$-tagging discriminant. For moderate and high $Z$-boson tagging efficiencies, the jet mass is the most discriminating of the three variables, but there is significant improvement in discrimination when combining all three inputs into a single tagger. At low $Z$-boson efficiencies, smaller than the $Z \rightarrow b \bar{b}$ branching ratio, the $b$-tagging discriminant is the most useful for rejecting $W$ bosons. The full tagger is largely unaffected by many systematic uncertainties on the inputs, with the exception of the uncertainties on the jet-mass scale and resolution. While it is not possible to measure the tagger efficiencies directly in data due to the lack of a pure sample of boosted, hadronically decaying $Z$ bosons, modelling of the likelihood function using hadronically decaying $W$ bosons has been studied in the data. Overall, the simulation agrees well with the $20.3 \mathrm{fb}^{-1}$ of $\sqrt{s}=8$ $\mathrm{TeV} p p$ data collected at the LHC.

Acknowledgments We thank CERN for the very successful operation of the LHC, as well as the support staff from our institutions without whom ATLAS could not be operated efficiently. We acknowledge the support of ANPCyT, Argentina; YerPhI, Armenia; ARC, Australia; BMWFW and FWF, Austria; ANAS, Azerbaijan; SSTC, Belarus; CNPq and FAPESP, Brazil; NSERC, NRC and CFI, Canada; CERN; CONICYT, Chile; CAS, MOST and NSFC, China; COLCIENCIAS, Colombia; MSMT CR, MPO CR and VSC CR, Czech Republic; DNRF, DNSRC and Lundbeck Foundation, Denmark; IN2P3-CNRS, CEADSM/IRFU, France; GNSF, Georgia; BMBF, HGF, and MPG, Germany; GSRT, Greece; RGC, Hong Kong SAR, China; ISF, I-CORE and Benoziyo Center, Israel; INFN, Italy; MEXT and JSPS, Japan; CNRST, Morocco; FOM and NWO, Netherlands; RCN, Norway; MNiSW and NCN, Poland; FCT, Portugal; MNE/IFA, Romania; MES of Russia and NRC KI, Russian Federation; JINR; MESTD, Serbia; MSSR, Slovakia; ARRS and MIZŠ, Slovenia; DST/NRF, South Africa; MINECO, Spain; SRC and Wallenberg Foundation, Sweden; SERI, SNSF and Cantons of Bern and Geneva, Switzerland; MOST, Taiwan; TAEK, Turkey; STFC, United Kingdom; DOE and NSF, United States of America. In addition, individual groups and members have received support from BCKDF, the Canada Council, CANARIE, CRC, Compute Canada, FQRNT, and the Ontario Innovation Trust, Canada; EPLANET, ERC, FP7, Horizon 2020 and Marie Sk?odowska-Curie Actions, European Union; Investissements d'Avenir Labex and Idex, ANR, Region Auvergne and Fondation Partager le Savoir, France; DFG and AvH Foundation, Germany; Herakleitos, Thales and Aristeia programmes co-financed by EU-ESF and the Greek NSRF; BSF, GIF and Minerva, Israel; BRF, Norway; the Royal Society and Leverhulme Trust, United Kingdom. The crucial computing support from all WLCG partners is acknowledged gratefully, in particular from CERN and the ATLAS Tier-1 facilities at TRIUMF (Canada), NDGF (Denmark, Norway, Sweden), CC-IN2P3 (France), KIT/GridKA (Germany), INFN-CNAF (Italy), NL-T1 (Netherlands), 
PIC (Spain), ASGC (Taiwan), RAL (UK) and BNL (USA) and in the Tier-2 facilities worldwide.

Open Access This article is distributed under the terms of the Creative Commons Attribution 4.0 International License (http://creativecomm ons.org/licenses/by/4.0/), which permits unrestricted use, distribution, and reproduction in any medium, provided you give appropriate credit to the original author(s) and the source, provide a link to the Creative Commons license, and indicate if changes were made.

Funded by SCOAP ${ }^{3}$.

\section{A. Correlations with 2-subjettiness}

The tagger developed in this paper is designed to work in conjunction with a procedure for separating generic quark and gluon jets from boson jets. Figure 19 shows the joint distribution of the jet mass and jet charge with a standard boson tagging variable 2 -subjettiness, $\tau_{21}$. The boson type tagger variables are nearly independent of $\tau_{21}$.
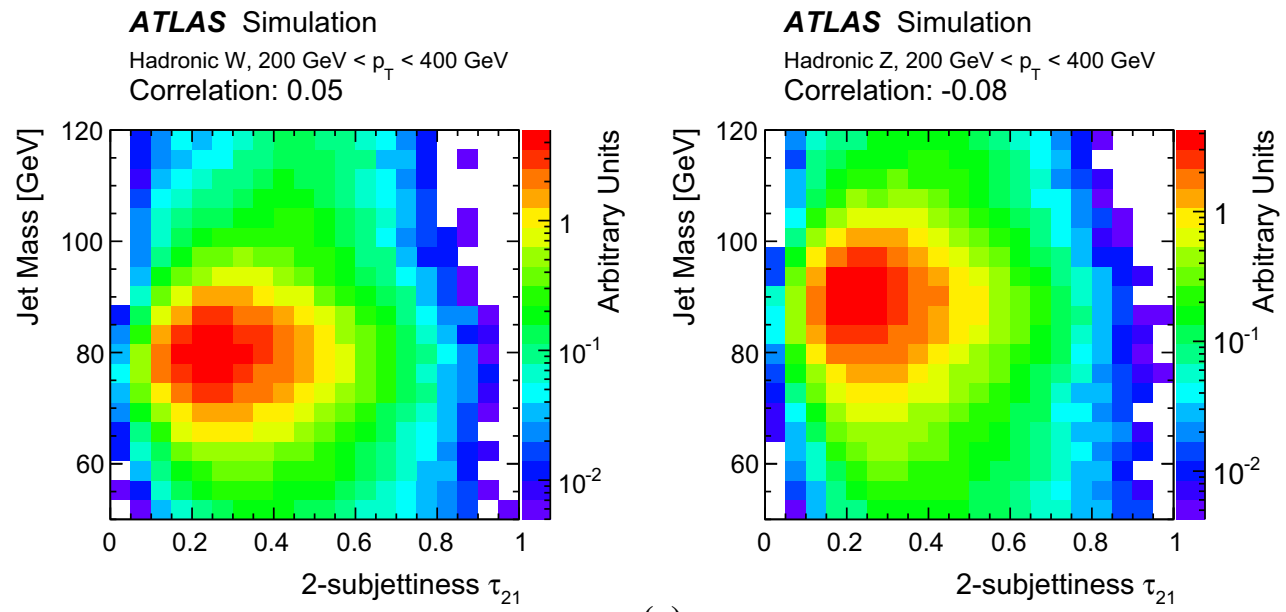

(a)
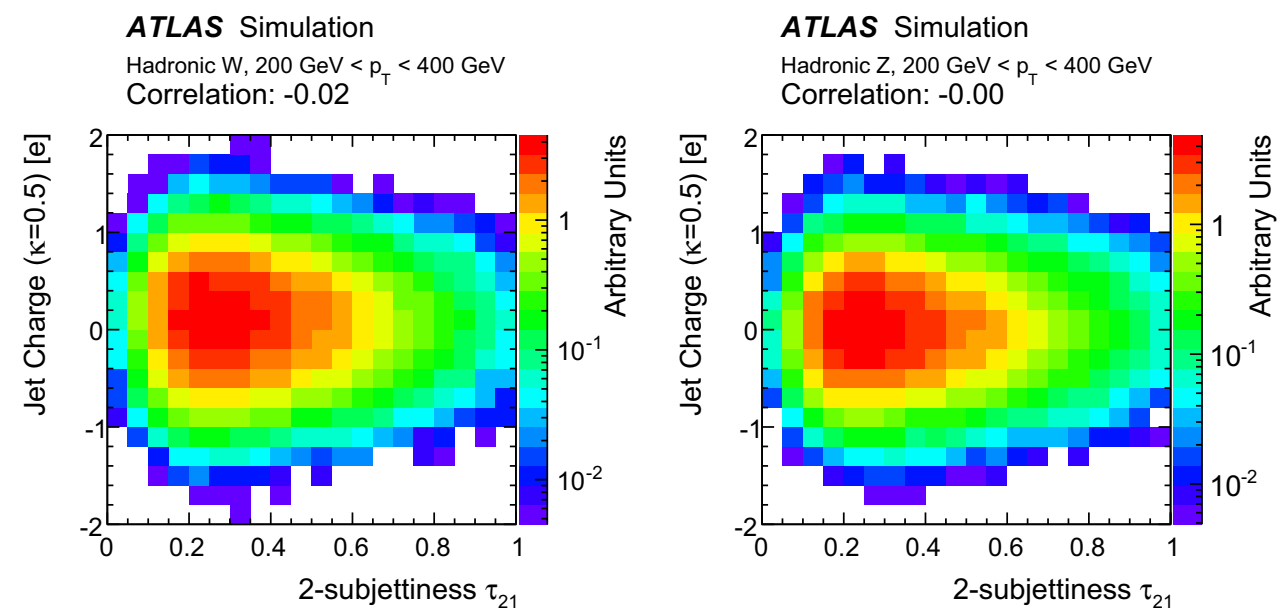

(b)

Fig. 19 The joint distribution of a jet mass and 2-subjettiness and $\mathbf{b}$ jet charge and 2-subjettiness for (left) $W$ boson jets and (right) $Z$ boson jets 


\section{References}

1. A. Altheimer et al., Boosted objects and jet substructure at the LHC. Report of BOOST2012, held at IFIC Valencia, 23rd-27th of July 2012. Eur. Phys. J. C 74, 2792 (2014). doi:10.1140/epjc/ s10052-014-2792-8. arXiv:1311.2708 [hep-ex]

2. ATLAS Collaboration, Performance of Boosted W Boson Identification with the ATLAS Detector (2014)

3. C.M.S. Collaboration, Identification techniques for highly boosted W bosons that decay into hadrons. JHEP 1412, 017 (2014). doi:10. 1007/JHEP12(2014)017. arXiv:1410.4227 [hep-ex]

4. S.L. Glashow, J. Iliopoulos, L. Maiani, Weak interactions with lepton-hadron symmetry. Phys. Rev. D 2, 1285-1292 (1970). doi:doi:10.1103/PhysRevD.2.1285

5. ATLAS Collaboration, A search for flavour changing neutral currents in top-quark decays in $p p$ collision data collected with the ATLAS detector at $\sqrt{s}=7$ TeV. JHEP 1209, 139 (2012). doi:10. 1007/JHEP09(2012)139. arXiv:1206.0257 [hep-ex]

6. CMS Collaboration, Search for flavor-changing neutral currents in top-quark decays $t \rightarrow Z q$ in $p p$ collisions at $\sqrt{s}=8 \mathrm{TeV}$. Phys. Rev. Lett. 112, 171802 (2014). doi:10.1103/PhysRevLett. 112.171802. arXiv:1312.4194 [hep-ex]

7. J. Thaler, K. Van Tilburg, Identifying boosted objects with N-subjettiness. JHEP 1103, 015 (2011). doi:10.1007/ JHEP03(2011)015. arXiv:1011.2268 [hep-ph]

8. T. Sjöstrand, S. Mrenna, P.Z. Skands, A brief introduction to PYTHIA 8.1. Comput. Phys. Commun. 178, 852-867 (2008). doi:10.1016/j.cpc.2008.01.036. arXiv:0710.3820 [hep-ph]

9. H.-L. Lai et al., New parton distributions for collider physics. Phys. Rev. D 82, 074024 (2010). doi:10.1103/PhysRevD.82.074024. arXiv:1007.2241 [hep-ph]

10. J. Gao et al., CT10 next-to-next-to-leading order global analysis of QCD. Phys. Rev. D 89, 033009 (2014). doi:10.1103/PhysRevD. 89.033009. arXiv:1302.6246 [hep-ph]

11. ATLAS Collaboration, Summary of ATLAS Pythia 8 Tunes. https:// cdsweb.cern.ch/record/1474107 (2012)

12. T. Sjöstrand, P.Z. Skands, Transverse-momentum-ordered showers and interleaved multiple interactions. Eur. Phys. J. C 39, 129-154 (2005). doi:10.1140/epjc/s2004-02084-y. arXiv:hep-ph/0408302 [hep-ph]

13. B. Andersson et al., Parton fragmentation and string dynamics. Phys. Rep. 97, 31 (1983). doi:10.1016/0370-1573(83)90080-7

14. M. Bähr et al., Herwig++ physics and manual. Eur. Phys. J. C 58, 639-707 (2008). doi:10.1140/epjc/s10052-008-0798-9. arXiv:0803.0883 [hep-ph]

15. S. Gieseke, P. Stephens, B. Webber, New formalism for QCD parton showers. JHEP 0312, 045 (2003). doi:10.1088/1126-6708/2003/ 12/045. arXiv:hep-ph

16. B. Webber, A QCD model for jet fragmentation including soft gluon interference. Nucl. Phys. B 238, 492 (1984). doi:10.1016/ 0550-3213(84)90333-X

17. S. Gieseke, C. Rohr, A. Siodmok, Colour reconnections in Herwig++. Eur. Phys. J. C 72, 2225 (2012). doi:10.1140/epjc/ s10052-012-2225-5. arXiv:1206.0041 [hep-ph]

18. P. Nason, A new method for combining NLO QCD with shower Monte Carlo algorithms. JHEP 0411, 040 (2004). doi:10.1088/ 1126-6708/2004/11/040. arXiv:hep-ph/0409146 [hep-ph]

19. S. Frixione, P. Nason, C. Oleari, Matching NLO QCD computations with Parton Shower simulations: the POWHEG method. JHEP 0711, 070 (2007). doi:10.1088/1126-6708/2007/11/070. arXiv:0709.2092 [hep-ph]

20. S. Alioli et al., A general framework for implementing NLO calculations in shower Monte Carlo programs: the POWHEG BOX. JHEP 1006, 043 (2010). doi:10.1007/JHEP06(2010)043. arXiv:1002.2581 [hep-ph]
21. T. Sjöstrand, S. Mrenna, P. Skands, PYTHIA 6.4 physics and manual. JHEP 0605, 026 (2006)

22. S. Frixione et al., Single-top hadroproduction in association with a W boson. JHEP 0807, 029 (2008). doi:10.1088/1126-6708/2008/ 07/029. arXiv:0805.3067 [hep-ph]

23. M.L. Mangano et al., ALPGEN, a generator for hard multiparton processes in hadronic collisions. JHEP 0307, 001 (2003). doi:10. 1088/1126-6708/2003/07/001. arXiv:hep-ph/0206293 [hep-ph]

24. J. Pumplin et al., New generation of parton distributions with uncertainties from global QCD analysis. JHEP 0207, 012 (2002). doi: 10. 1088/1126-6708/2002/07/012. arXiv:hep-ph/0201195 [hep-ph]

25. G. Corcella et al., HERWIG 6.5 release note (2002). arXiv:hep-ph/0210213 [hep-ph]

26. ATLAS Collaboration, New ATLAS event generator tunes to 2010 data. ATL-PHYS-PUB-2011-008 (2011). https://cds.cern. ch/record/1345343

27. P.Z. Skands, Tuning Monte Carlo generators: the Perugia tunes. Phys. Rev. D 82, 074018 (2010). doi:10.1103/PhysRevD.82. 074018. arXiv: 1005.3457 [hep-ph]

28. ATLAS Collaboration, The ATLAS simulation infrastructure. Eur. Phys. J. C 70, 823 (2010). doi:10.1140/epjc/s10052-010-1429-9. arXiv: 1005.4568 [hep-ex]

29. S. Agostinelli et al., GEANT4 Collaboration, Geant4-a simulation toolkit. Nucl. Instrum. Meth. A 506, 250 (2003). doi:10.1016/ S0168-9002(03)01368-8

30. K.A. Olive et al., Particle Data Group, The review of particle physics. Chin. Phys. C 38, 090001 (2014). doi:10.1088/1674-1137/ 38/9/090001

31. N. Cabibbo, Unitary symmetry and leptonic decays. Phys. Rev. Lett. 10, 531-533 (1963). doi:10.1103/PhysRevLett.10.531

32. M. Kobayashi, T. Maskawa, CP violation in the renormalizable theory of weak interaction. Prog. Theor. Phys. 49, 652-657 (1973). doi:10.1143/PTP.49.652

33. ATLAS Collaboration, The ATLAS experiment at the CERN Large Hadron Collider. JINST 3, S08003 (2008). doi:10.1088/ 1748-0221/3/08/S08003

34. ATLAS Collaboration, Calorimeter Clustering Algorithms: Description and Performance (2008). https://cdsweb.cern.ch/ record/1099735

35. C. Cojocaru et al., Hadronic calibration of the ATLAS liquid argon end-cap calorimeter in the pseudorapidity region $1.6<|\eta|>1.8$ in beam tests. Nucl. Instrum. Meth. A 531, 481 (2004). doi:10. 1016/j.nima.2004.05.133. arXiv:physics/0407009

36. ATLAS Collaboration, Local Hadronic Calibration (2009). https:// cdsweb.cern.ch/record/1112035

37. M. Cacciari, G.P. Salam, G. Soyez, The anti-k(t) jet clustering algorithm. JHEP 0804, 063 (2008). doi:10.1088/1126-6708/2008/04/ 063. arXiv:0802.1189 [hep-ph]

38. D. Krohn, J. Thaler, L.-T. Wang, Jet trimming. JHEP 1002, 084 (2010). doi:10.1007/JHEP02(2010)084. arXiv:0912.1342 [hep$\mathrm{ph}]$

39. ATLAS Collaboration, Pile-up subtraction and suppression for jets in ATLAS. ATLAS-CONF-2013-083 (2013). https://cdsweb.cern. $\mathrm{ch} /$ record/1570994

40. M. Cacciari, G.P. Salam, G. Soyez, The catchment area of jets. JHEP 0804, 005 (2008). doi:10.1088/1126-6708/2008/04/005. arXiv:0802.1188 [hep-ph]

41. R.D. Field, R.P. Feynman, A parameterization of the properties of quark jets. Nucl. Phys. B 136, 1 (1978). doi:10.1016/ 0550-3213(78)90015-9

42. ATLAS Collaboration, Jet charge studies with the ATLAS detector using $\sqrt{s}=8 \mathrm{TeV}$ proton-proton collision data (2013). https:// cdsweb.cern.ch/record/1572980 
43. ATLAS Collaboration, Calibration of b-tagging using dileptonic top pair events in a combinatorial likelihood approach with the ATLAS experiment (2014). https://cdsweb.cern.ch/record/ 1664335

44. ATLAS Collaboration, Calibration of the performance of b-tagging for c and light-flavour jets in the 2012 ATLAS data (2014). https:// cdsweb.cern.ch/record/ 1741020

45. ATLAS Collaboration, Performance of jet substructure techniques for large-R jets in proton-proton collisions at $\sqrt{s}=7 \mathrm{TeV}$ using the ATLAS detector. JHEP 1309, 076 (2013). doi:10.1007/ JHEP09(2013)076. arXiv:1306.4945 [hep-ex]

46. ATLAS Collaboration, Jet mass and substructure of inclusive jets in $\sqrt{s}=7 \mathrm{TeV}$ pp collisions with the ATLAS experiment. JHEP 1205, 128 (2012). doi:10.1007/JHEP05(2012)128. arXiv:1203.4606 [hep-ex]

47. ATLAS Collaboration, Charged-particle multiplicities in pp interactions measured with the ATLAS detector at the LHC. N. J. Phys. 13, 053033 (2011). doi:10.1088/1367-2630/13/5/053033. arXiv:1012.5104 [hep-ex]
48. ATLAS Collaboration, Measurements of normalized differential cross sections for $t \bar{t}$ production in pp collisions at $\sqrt{s}=7 \mathrm{TeV}$ using the ATLAS detector. Phys. Rev. D 90, 072004 (2014). doi:10. 1103/PhysRevD.90.072004. arXiv:1407.0371 [hep-ex]

49. CMS Collaboration, Measurement of masses in the $t \bar{t}$ system by kinematic endpoints in pp collisions at $\sqrt{s}=7 \mathrm{TeV}$. Eur. Phys. J. C 73, 2494 (2013). doi:10.1140/epjc/s10052-013-2494-7. arXiv: 1304.5783 [hep-ex]

50. ATLAS Collaboration, Light-quark and gluon jet discrimination in pp collisions at $\sqrt{s}=7 \mathrm{TeV}$ with the ATLAS detector. Eur. Phys. J. C 74, 3023 (2014). doi:10.1140/epjc/s10052-014-3023-z. arXiv: 1405.6583 [hep-ex]

51. ATLAS Collaboration, Measurement of the WW + WZ cross section and limits on anomalous triple gauge couplings using final states with one lepton, missing transverse momentum, and two jets with the ATLAS detector at $\sqrt{\mathrm{s}}=7 T e V$. JHEP 1501, 049 (2015). doi:10.1007/JHEP01(2015)049. arXiv:1410.7238 [hep-ex]

\section{ATLAS Collaboration}

G. Aad ${ }^{85}$, B. Abbott ${ }^{113}$, J. Abdallah ${ }^{151}$, O. Abdinov ${ }^{11}$, R. Aben ${ }^{107}$, M. Abolins ${ }^{90}$, O. S. AbouZeid ${ }^{158}$, H. Abramowicz ${ }^{153}$, H. Abreu ${ }^{152}$, R. Abreu ${ }^{116}$, Y. Abulaiti ${ }^{146 a, 146 b}$, B. S. Acharya ${ }^{164 a, 164 b, a}$, L. Adamczyk ${ }^{38 a}$, D. L. Adams ${ }^{25}$, J. Adelman ${ }^{108}$, S. Adomeit ${ }^{100}$, T. Adye ${ }^{131}$, A. A. Affolder ${ }^{74}$, T. Agatonovic-Jovin ${ }^{13}$, J. Agricola ${ }^{54}$, J. A. Aguilar-Saavedra ${ }^{126 a, 126 f}$,

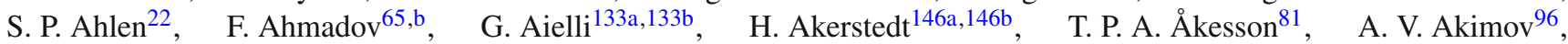
G. L. Alberghi ${ }^{20 a, 20 b}$, J. Albert ${ }^{169}$, S. Albrand ${ }^{55}, \quad$ M. J. Alconada Verzini ${ }^{71}, \quad$ M. Aleksa ${ }^{30}$, I. N. Aleksandrov ${ }^{65}$, $\begin{array}{llll}\text { C. Alexa } & \text { 26a } & \text { G. Alexander } & 153\end{array}$ T. Alexopoulos ${ }^{10}, \quad$ M. Alhroob ${ }^{113}, \quad$ G. Alimonti ${ }^{91 a}$, L. Alio ${ }^{85}, \quad$ J. Alison ${ }^{31}$, S. P. Alkire ${ }^{35}$, B. M. M. Allbrooke ${ }^{149}$, P. P. Allport ${ }^{74}$, A. Aloisio104a,104b , A. Alonso ${ }^{36}$, F. Alonso ${ }^{71}$, C. Alpigiani ${ }^{76}$, A. Altheimer ${ }^{35}$, B. Alvarez Gonzalez ${ }^{30}$, D. Álvarez Piqueras ${ }^{167}$, M. G. Alviggi 104a, 104b , B. T. Amadio ${ }^{15}$, K. Amako ${ }^{66}$, Y. Amaral Coutinho ${ }^{24 a}$, C. Amelung ${ }^{23}$, D. Amidei ${ }^{89}$, S. P. Amor Dos Santos 126a,126c , A. Amorim ${ }^{126 a, 126 b}$, S. Amoroso ${ }^{48}$, N. $A \operatorname{Aram}^{153}$, G. Amundsen ${ }^{23}$, C. Anastopoulos ${ }^{139}$, L. S. Ancu ${ }^{49}$, N. Andari ${ }^{108}$, T. Andeen ${ }^{35}$, C. F. Anders ${ }^{58 b}$, G. Anders ${ }^{30}$, J. K. Anders ${ }^{74}$, K. J. Anderson ${ }^{31}$ ， A. Andreazza ${ }^{91 a, 91 b}$, V. Andrei ${ }^{58 a}$ ， S. Angelidakis ${ }^{9}$ ， I. Angelozzi ${ }^{107}$ ， P. Anger ${ }^{44}$, A. Angerami ${ }^{35}$, F. Anghinolfi ${ }^{30}$, A. V. Anisenkov ${ }^{109, c}$, N. Anjos ${ }^{12}$, A. Annovi ${ }^{124 a, 124 b}$, M. Antonelli4 ${ }^{47}$, A. Antonov ${ }^{98}$, J. Antos ${ }^{144 b}$, F. Anulli ${ }^{132 a}$, M. Aoki ${ }^{66}$, L. Aperio Bella ${ }^{18}$, G. Arabidze ${ }^{90}$, Y. Arai ${ }^{66}$, J. P. Araque ${ }^{126 a}$, A. T. H. Arce ${ }^{45}$, F. A. Arduh ${ }^{71}$, J-F. Arguin ${ }^{95}$, S. Argyropoulos ${ }^{63}$, M. Arik ${ }^{19 a}$, A. J. Armbruster ${ }^{30}$, O. Arnaez ${ }^{30}$, V. Arnal ${ }^{82}$, H. Arnold ${ }^{48}$, M. Arratia ${ }^{28}$, O. Arslan ${ }^{21}$, A. Artamonov ${ }^{97}$, G. Artoni ${ }^{23}$, S. Asai ${ }^{155}$, N. Asbah ${ }^{42}$, A. Ashkenazi ${ }^{153}$, B. Åsman ${ }^{146 a, 146 b}$, L. Asquith ${ }^{149}$ ，K. Assamagan ${ }^{25}$ ， R. Astalos ${ }^{144 a}$ ， M. Atkinson ${ }^{165}$ ， N. B. Atlay ${ }^{141}$ ， K. Augsten ${ }^{128}$ ， M. Aurousseau ${ }^{145 b}$, G. Avolio ${ }^{30}$, B. Axen ${ }^{15}$, M. K. Ayoub ${ }^{117}$, G. Azuelos ${ }^{95, \text { d }, \text { M. A. Baak }}{ }^{30}$, A. E. Baas ${ }^{58 a}$, M. J. Baca ${ }^{18}$, C. Bacci ${ }^{134 a, 134 b}$, H. Bachacou ${ }^{136}$, K. Bachas ${ }^{154}$, M. Backes ${ }^{30}$, M. Backhaus ${ }^{30}$, P. Bagiacchi ${ }^{132 a, 132 b}$, P. Bagnaia ${ }^{132 a, 132 b}$, Y. Bai $^{33 a}$, T. Bain ${ }^{35}$, J. T. Baines ${ }^{131}$, O. K. Baker ${ }^{176}$, E. M. Baldin ${ }^{109, \mathrm{c}}$, P. Balek ${ }^{129}$, T. Balestri ${ }^{148}$, F. Balli ${ }^{84}$, W. K. Balunas ${ }^{122}$, E. Banas ${ }^{39}$, Sw. Banerjee ${ }^{173}$, A. A. E. Bannoura ${ }^{175}$, H. S. Bansil ${ }^{18}$, L. Barak ${ }^{30}$, E. L. Barberio ${ }^{88}$, D. Barberis ${ }^{50 a, 50 b}$, M. Barbero ${ }^{85}$, T. Barillari ${ }^{101}$ ， M. Barisonzi ${ }^{164 a, 164 b}$ ，T. Barklow ${ }^{143}$ ， N. Barlow ${ }^{28}$ ，S. L. Barnes ${ }^{84}$ ，B. M. Barnett ${ }^{131}$ ， R. M. Barnett ${ }^{15}$, Z. Barnovska ${ }^{5}$, A. Baroncelli134a， G. Barone ${ }^{23}$, A. J. Barr ${ }^{120}$, F. Barreiro ${ }^{82}$, J. Barreiro Guimarães da Costa ${ }^{57}$, R. Bartoldus ${ }^{143}$, A. E. Barton ${ }^{72}$, P. Bartos ${ }^{144 a}$, A. Basalaev ${ }^{123}$, A. Bassalat ${ }^{117}$, A. Basye ${ }^{165}$, R. L. Bates ${ }^{53}$, S. J. Batista ${ }^{158}$, J. R. Batley ${ }^{28}$, M. Battaglia ${ }^{137}$, M. Bauce ${ }^{132 a, 132 b}$, F. Bauer ${ }^{136}$, H. S. Bawa ${ }^{143, e}$, J. B. Beacham ${ }^{111}$, M. D. Beattie ${ }^{72}$, T. Beau ${ }^{80}$ ， P. H. Beauchemin ${ }^{161}$ ， R. Beccherle 124a,124b , P. Bechtle ${ }^{21}$ ， H. P. Beck ${ }^{17, f}$ ， K. Becker ${ }^{120}, \quad$ M. Becker ${ }^{83}$, M. Beckingham ${ }^{170}$, C. Becot ${ }^{117}$ ，A. J. Beddall ${ }^{19 b}$ ，A. Beddall ${ }^{19 b}$ ，V. A. Bednyakov ${ }^{65}$ ，C. P. Bee ${ }^{148}$ ， L. J. Beemster ${ }^{107}$, T. A. Beermann ${ }^{30}$, M. Begel ${ }^{25}$, J. K. Behr ${ }^{120}$, C. Belanger-Champagne ${ }^{87}$, W. H. Bell ${ }^{49}$, G. Bella ${ }^{153}$, L. Bellagamba ${ }^{20 a}$, A. Bellerive ${ }^{29}$, M. Bellomo ${ }^{86}$, K. Belotskiy ${ }^{98}$, O. Beltramello ${ }^{30}$, O. Benary ${ }^{153}$, D. Benchekroun ${ }^{135 a}$, M. Bender ${ }^{100}$, K. Bendtz ${ }^{146 a, 146 b}$, N. Benekos ${ }^{10}$, Y. Benhammou ${ }^{153}$, E. Benhar Noccioli ${ }^{49}$, J. A. Benitez Garcia ${ }^{159 b}$, D. P. Benjamin ${ }^{45}$, J. R. Bensinger ${ }^{23}$, S. Bentvelsen ${ }^{107}$, L. Beresford ${ }^{120}$, M. Beretta ${ }^{47}$, D. Berge ${ }^{107}$, E. Bergeaas Kuutmann ${ }^{166}$, N. Berger ${ }^{5}$, F. Berghaus ${ }^{169}$, J. Beringer ${ }^{15}$, C. Bernard ${ }^{22}$, N. R. Bernard ${ }^{86}$, C. Bernius ${ }^{110}$, F. U. Bernlochner ${ }^{21}$, T. Berry ${ }^{77}$, P. Berta ${ }^{129}$, C. Bertella ${ }^{83}$, G. Bertoli 146a,146b, F. Bertolucci ${ }^{124 a, 124 b}$, C. Bertsche ${ }^{113}$, D. Bertsche ${ }^{113}$, M. I. Besana ${ }^{91 a}$, G. J. Besjes ${ }^{36}$, O. Bessidskaia Bylund ${ }^{146 a, 146 b}$, M. Bessner ${ }^{42}$, N. Besson ${ }^{136}$, C. Betancourt ${ }^{48}$, S. Bethke ${ }^{101}$, A. J. Bevan ${ }^{76}$, W. Bhimji ${ }^{15}$, 
R. M. Bianchi ${ }^{125}$ ， L. Bianchini ${ }^{23} ，$ M. Bianco $^{30}$ ， O. Biebel ${ }^{100 ， \quad D . \text { Biedermann }^{16} \text { ， S. P. Bieniek }}{ }^{78}$ ， M. Biglietti ${ }^{134 a}$, J. Bilbao De Mendizabal ${ }^{49}$, H. Bilokon ${ }^{47}$, M. Bindi ${ }^{54}$, S. Binet ${ }^{117}$, A. Bingul ${ }^{19 b}$, C. Bini ${ }^{132 a, 132 b}$, S. Biondi ${ }^{20 a, 20 b}$, C. W. Black ${ }^{150}$, J. E. Black ${ }^{143}$, K. M. Black ${ }^{22}$, D. Blackburn ${ }^{138}$, R. E. Blair ${ }^{6}$, J.-B. Blanchard ${ }^{136}$, J. E. Blanco ${ }^{77}$, T. Blazek ${ }^{144 a}$ ， I. Bloch ${ }^{42}$ ， C. Blocker ${ }^{23}$ ，W. Blum ${ }^{83, *}$ ，U. Blumenschein ${ }^{54}$, G. J. Bobbink ${ }^{107}$ ， V. S. Bobrovnikov ${ }^{109, c}$, S. S. Bocchetta ${ }^{81}$, A. Bocci ${ }^{45}$, C. Bock ${ }^{100}$, M. Boehler ${ }^{48}$, J. A. Bogaerts ${ }^{30}$, D. Bogavac ${ }^{13}$, A. G. Bogdanchikov ${ }^{109}$, C. Bohm ${ }^{146 a}$, V. Boisvert ${ }^{77}$, T. Bold ${ }^{38 a}$, V. Boldea ${ }^{26 a}$, A. S. Boldyrev ${ }^{99}$, M. Bomben ${ }^{80}$, M. Bona ${ }^{76}$, M. Boonekamp ${ }^{136}$, A. Borisov ${ }^{130}$, G. Borissov ${ }^{72}, \quad$ S. Borroni ${ }^{42}$, J. Bortfeldt ${ }^{100}$, V. Bortolotto ${ }^{60 a, 60 b, 60 c}, \quad$ K. Bos ${ }^{107}, \quad$ D. Boscherini ${ }^{20 a}$, M. Bosman ${ }^{12}$, J. Boudreau ${ }^{125}$, J. Bouffard ${ }^{2}$, E. V. Bouhova-Thacker ${ }^{72}$, D. Boumediene ${ }^{34}$, C. Bourdarios ${ }^{117}$, N. Bousson ${ }^{114}$, A. Boveia ${ }^{30}$, J. Boyd ${ }^{30}$, I. R. Boyko ${ }^{65}$, I. Bozic ${ }^{13}$, J. Bracinik ${ }^{18}$, A. Brandt ${ }^{8}$, G. Brandt ${ }^{54}$, O. Brandt ${ }^{58 a}$, U. Bratzler ${ }^{156}$, B. Brau ${ }^{86}$, J. E. Brau ${ }^{116}$, H. M. Braun ${ }^{175, *}$ S. F. Brazzale ${ }^{164 a, 164 c}$, W. D. Breaden Madden ${ }^{53}$, K. Brendlinger ${ }^{122}$, A. J. Brennan $^{88}$, L. Brenner ${ }^{107}$, R. Brenner ${ }^{166}, \quad$ S. Bressler ${ }^{172}$, K. Bristow ${ }^{145 c}$, T. M. Bristow ${ }^{46}, \quad$ D. Britton ${ }^{53}$, D. Britzger ${ }^{42}$, F. M. Brochu ${ }^{28}$, I. Brock ${ }^{21}$, R. Brock ${ }^{90}$, J. Bronner ${ }^{101}$, G. Brooijmans ${ }^{35}$, T. Brooks ${ }^{77}$, W. K. Brooks ${ }^{32 \mathrm{~b}}$, J. Brosamer ${ }^{15}$, E. Brost ${ }^{116}$, J. Brown ${ }^{55}$, P. A. Bruckman de Renstrom ${ }^{39}$, D. Bruncko ${ }^{144 b}$, R. Bruneliere ${ }^{48}$, A. Bruni ${ }^{20 a}$, G. Bruni ${ }^{20 a}$, M. Bruschi ${ }^{20 a}$, N. Bruscino ${ }^{21}$, L. Bryngemark ${ }^{81}$, T. Buanes ${ }^{14}$, Q. Buat ${ }^{142}$, P. Buchholz ${ }^{141}$, A. G. Buckley ${ }^{53}$, S. I. Buda ${ }^{26 a}$, I. A. Budagov ${ }^{65}$, F. Buehrer ${ }^{48}$, L. Bugge ${ }^{119}$, M. K. Bugge ${ }^{119}$, O. Bulekov98, D. Bullock ${ }^{8}$, H. Burckhart ${ }^{30}$, S. Burdin ${ }^{74}$, C. D. Burgard ${ }^{48}$, B. Burghgrave ${ }^{108}$, S. Burke ${ }^{131}$, I. Burmeister ${ }^{43}$, E. Busato ${ }^{34}$, D. Büscher ${ }^{48}$, V. Büscher ${ }^{83}$, P. Bussey ${ }^{53}$, J. M. Butler ${ }^{22}$, A. I. Butt ${ }^{3}$, C. M. Buttar ${ }^{53}$, J. M. Butterworth ${ }^{78}$, P. Butti ${ }^{107}$, W. Buttinger ${ }^{25}$, A. Buzatu ${ }^{53}$, A. R. Buzykaev ${ }^{109, c}$, S. Cabrera Urbán ${ }^{167}$, D. Caforio ${ }^{128}$, V. M. Cairo ${ }^{37 a, 37 b}$, O. Cakir ${ }^{4 a}$, N. Calace ${ }^{49}$, P. Calafiura ${ }^{15}$,

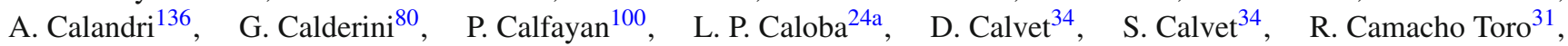

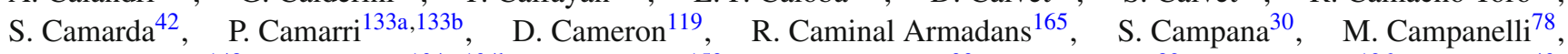
A. Campoverde ${ }^{148}$, V. Canale ${ }^{104 a, 104 b}$, A. Canepa ${ }^{159 a}, \quad$ M. Cano Bret ${ }^{33 e}, \quad J$. Cantero $^{82}, \quad$ R. Cantrill126a, T. Cao ${ }^{40}$, M. D. M. Capeans Garrido ${ }^{30}$, I. Caprini ${ }^{26 a}$, M. Caprini ${ }^{26 a}$, M. Capua ${ }^{37 a, 37 b}$, R. Caputo ${ }^{83}$, R. Cardarelli ${ }^{133 a}$, F. Cardillo ${ }^{48}$, T. Carli ${ }^{30}$, G. Carlino ${ }^{104 a}$, L. Carminati ${ }^{91 a, 91 b}$, S. Caron ${ }^{106}$, E. Carquin ${ }^{32 a}$, G. D. Carrillo-Montoya ${ }^{30}$, J. R. Carter ${ }^{28}$,

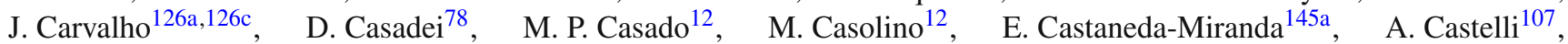
V. Castillo Gimenez ${ }^{167}$, N. F. Castro ${ }^{126 a, g}$, P. Catastini ${ }^{57}$, A. Catinaccio ${ }^{30}$, J. R. Catmore ${ }^{119}$, A. Cattai ${ }^{30}$, J. Caudron ${ }^{83}$,

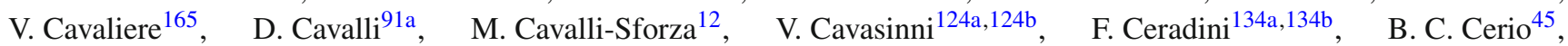
K. Cerny ${ }^{129}$, A. S. Cerqueira ${ }^{24 b}$, A. Cerri ${ }^{149}$, L. Cerrito ${ }^{76}$, F. Cerutti ${ }^{15}$, M. Cerv ${ }^{30}$, A. Cervelli ${ }^{17}$, S. A. Cetin ${ }^{19 c}$, A. Chafaq ${ }^{135 a}$, D. Chakraborty ${ }^{108}$, I. Chalupkova ${ }^{129}$, P. Chang ${ }^{165}$, J. D. Chapman ${ }^{28}$, D. G. Charlton ${ }^{18}$, C. C. Chau ${ }^{158}$, C. A. Chavez Barajas ${ }^{149}$, S. Cheatham ${ }^{152}$, A. Chegwidden ${ }^{90}$, S. Chekanov $^{6}$, S. V. Chekulaev ${ }^{159 a}$, G. A. Chelkov ${ }^{65, h}$, M. A. Chelstowska ${ }^{89}$, C. Chen ${ }^{64}$, H. Chen ${ }^{25}$, K. Chen ${ }^{148}$, L. Chen ${ }^{33 d, i}$, S. Chen ${ }^{33 c}$, X. Chen ${ }^{33 f}$, Y. Chen ${ }^{67}$, H. C. Cheng ${ }^{89}$, Y. Cheng ${ }^{31}$, A. Cheplakov ${ }^{65}$, E. Cheremushkina ${ }^{130}$, R. Cherkaoui El Moursli ${ }^{135 e}$, V. Chernyatin ${ }^{25, *}$ E. Cheu ${ }^{7}$, L. Chevalier ${ }^{136}$, V. Chiarella ${ }^{47}$, G. Chiarelli ${ }^{124 a, 124 b}$, G. Chiodini ${ }^{73 a}$, A. S. Chisholm ${ }^{18}$, R. T. Chislett $^{78}$, A. Chitan ${ }^{26 a}$,

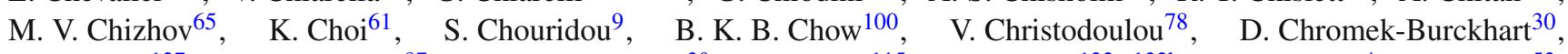
J. Chudoba ${ }^{127}$, A. J. Chuinard ${ }^{87}$, J. J. Chwastowski ${ }^{39}$, L. Chytka ${ }^{115}$, G. Ciapetti ${ }^{132 a, 132 b}$, A. K. Ciftci ${ }^{4 a}$, D. Cinca ${ }^{53}$, V. Cindro $^{75}$, I. A. Cioara ${ }^{21}$, A. Ciocio ${ }^{15}$, F. Cirotto ${ }^{104 a, 104 b}$, Z. H. Citron ${ }^{172}$, M. Ciubancan ${ }^{26 a}$, A. Clark ${ }^{49}$, B. L. Clark ${ }^{57}$,

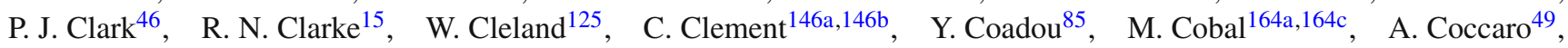
J. Cochran ${ }^{64}$, L. Coffey ${ }^{23}$, J. G. Cogan ${ }^{143}$, L. Colasurdo ${ }^{106}, \quad$ B. Cole ${ }^{35}, \quad$ S. Cole ${ }^{108}$, A. P. Colijn ${ }^{107}$, J. Collot ${ }^{55}$, T. Colombo ${ }^{58 c}$, G. Compostella ${ }^{101}$, P. Conde Muiño ${ }^{126 a, 126 b}$, E. Coniavitis ${ }^{48}$, S. H. Connell ${ }^{145 b}$, I. A. Connelly ${ }^{77}$, V. Consorti ${ }^{48}$, S. Constantinescu ${ }^{26 a}$, C. Conta ${ }^{121 a, 121 b}, \quad$ G. Conti ${ }^{30}$, F. Conventi ${ }^{104 a, j}$, M. Cooke ${ }^{15}$, B. D. Cooper ${ }^{78}$, A. M. Cooper-Sarkar ${ }^{120}$, T. Cornelissen ${ }^{175}$, M. Corradi ${ }^{20 a}$, F. Corriveau ${ }^{87, k}$, A. Corso-Radu ${ }^{163}$, A. Cortes-Gonzalez ${ }^{12}$, G. Cortiana ${ }^{101}$, G. Costa ${ }^{91 a}$, M. J. Costa ${ }^{167}$, D. Costanzo $^{139}$, D. Côté ${ }^{8}$, G. Cottin ${ }^{28}$, G. Cowan ${ }^{77}$, B. E. Cox ${ }^{84}$, K. Cranmer ${ }^{110}$, G. Cree ${ }^{29}$, S. Crépé-Renaudinn ${ }^{55}$ F. Crescioli ${ }^{80}$, W. A. Cribbs ${ }^{146 a, 146 b}, \quad$ M. Crispin Ortuzar ${ }^{120}, \quad$ M. Cristinziani ${ }^{21}$, V. Croft ${ }^{106}$, G. Crosetti ${ }^{37 a, 37 b}$, T. Cuhadar Donszelmann ${ }^{139}$, J. Cummings ${ }^{176}, \quad$ M. Curatolo ${ }^{47}, \quad$ C. Cuthbert ${ }^{150}$, H. Czirr ${ }^{141}$, P. Czodrowski ${ }^{3}$, S. D’Auria ${ }^{53}$, M. D’Onofrio ${ }^{74}$, M. J. Da Cunha Sargedas De Sousa ${ }^{126 a, 126 b}$, C. Da Via ${ }^{84}$, W. Dabrowski ${ }^{38 a}$, A. Dafinca ${ }^{120}$, T. Dai ${ }^{89}$, O. Dale ${ }^{14}$, F. Dallaire ${ }^{95}$, C. Dallapiccola ${ }^{86}$, M. Dam ${ }^{36}$, J. R. Dandoy ${ }^{31}$, N. P. Dang ${ }^{48}$, A. C. Daniells ${ }^{18}$, M. Danninger ${ }^{168}$, M. Dano Hoffmann ${ }^{136}$, V. Dao ${ }^{48}, \quad$ G. Darbo ${ }^{50 a}, \quad$ S. Darmora ${ }^{8}$, J. Dassoulas ${ }^{3}$, A. Dattagupta ${ }^{61}$, W. Davey ${ }^{21}$, C. David ${ }^{169}$, T. Davidek ${ }^{129}$, E. Davies ${ }^{120,1}$, M. Davies ${ }^{153}$, P. Davison ${ }^{78}$, Y. Davygora ${ }^{58 a}$, E. Dawe ${ }^{88}$, I. Dawson ${ }^{139}$, R. K. Daya-Ishmukhametova ${ }^{86}$, K. De ${ }^{8}$, R. de Asmundis ${ }^{104 a}$, A. De Benedetti ${ }^{113}$, S. De Castro ${ }^{20 a, 20 b}$, S. De Cecco ${ }^{80}$, N. De Groot ${ }^{106}$, P. de Jong ${ }^{107}$, H. De la Torre ${ }^{82}$, F. De Lorenzi ${ }^{64}$, D. De Pedis ${ }^{132 a}$, A. De Salvo ${ }^{132 a}$, U. De Sanctis ${ }^{149}$, A. De Santo ${ }^{149}$, J. B. De Vivie De Regie ${ }^{117}$, W. J. Dearnaley ${ }^{72}$, R. Debbe ${ }^{25}$, C. Debenedetti ${ }^{137}$, D. V. Dedovich ${ }^{65}$, I. Deigaard ${ }^{107}$, J. Del Peso ${ }^{82}$, T. Del Prete ${ }^{124 a, 124 b}$, D. Delgove ${ }^{117}$, F. Deliot ${ }^{136}$, C. M. Delitzsch ${ }^{49}$, M. Deliyergiyev ${ }^{75}$, A. Dell'Acqua ${ }^{30}$, L. Dell'Asta ${ }^{22}$, M. Dell'Orso ${ }^{124 a, 124 b}$, M. Della Pietra 104a,j, D. della Volpe ${ }^{49}$, M. Delmastro ${ }^{5}$, P. A. Delsart ${ }^{55}$, C. Deluca ${ }^{107}$, D. A. DeMarco ${ }^{158}$, S. Demers ${ }^{176}$, M. Demichev ${ }^{65}$, 
A. Demilly ${ }^{80}$, S. P. Denisov ${ }^{130}$, D. Derendarz ${ }^{39}$, J. E. Derkaoui ${ }^{135 d}$, F. Derue ${ }^{80}$, P. Dervan ${ }^{74}$, K. Desch ${ }^{21}$, C. Deterre ${ }^{42}$, P. O. Deviveiros ${ }^{30}$, A. Dewhurst ${ }^{131}$ ， S. Dhaliwal ${ }^{23}$ ， A. Di Ciaccio ${ }^{133 a, 133 b}$ ， L. Di Ciaccio ${ }^{5}$ ． A. Di Domenico ${ }^{132 a, 132 b}$,

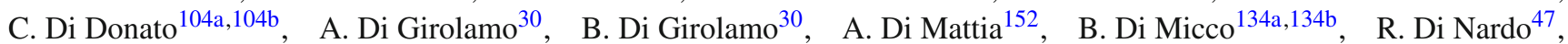

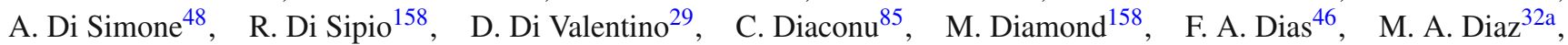
E. B. Diehl ${ }^{89}$, J. Dietrich ${ }^{16}$, S. Diglio ${ }^{85}$, A. Dimitrievska ${ }^{13}$, J. Dingfelder ${ }^{21}$, P. Dita ${ }^{26 a}$, S. Dita ${ }^{26 a}$, F. Dittus ${ }^{30}$, F. Djama ${ }^{85}$, T. Djobava ${ }^{51 b}$, J. I. Djuvsland ${ }^{58 a}$, M. A. B. do Vale ${ }^{24 c}$, D. Dobos ${ }^{30}$, M. Dobre ${ }^{26 a}$, C. Doglioni ${ }^{81}$, T. Dohmae ${ }^{155}$, J. Dolejsi ${ }^{129}$, Z. Dolezal ${ }^{129}$, B. A. Dolgoshein ${ }^{98, *}$, M. Donadelli24d, S. Donati ${ }^{124 a, 124 b}$, P. Dondero ${ }^{121 a, 121 b}$, J. Donini ${ }^{34}$, J. Dopke ${ }^{131}$, A. Doria ${ }^{104 a}$, M. T. Dova ${ }^{71}$, A. T. Doyle ${ }^{53}$, E. Drechsler ${ }^{54}$, M. Dris ${ }^{10}$, E. Dubreuil ${ }^{34}$, E. Duchovni ${ }^{172}$, G. Duckeck ${ }^{100}$, O. A. Ducu ${ }^{26 a}, 85$, D. Duda ${ }^{107}$, A. Dudarev ${ }^{30}$, L. Duflot ${ }^{117}$, L. Duguid ${ }^{77}$, M. Dührssen ${ }^{30}$, M. Dunford ${ }^{58 a}$, H. Duran Yildiz ${ }^{4 a}$, M. Düren ${ }^{52}$, A. Durglishvili ${ }^{51 b}$, D. Duschinger ${ }^{44}$, M. Dyndal ${ }^{38 a}$, C. Eckardt ${ }^{42}$, K. M. Ecker ${ }^{101}$, R. C. Edgar ${ }^{89}$, W. Edson ${ }^{2}$, N. C. Edwards ${ }^{46}$, W. Ehrenfeld ${ }^{21}$, T. Eifert ${ }^{30}$, G. Eigen $^{14}$, K. Einsweiler ${ }^{15}$, T. Ekelof ${ }^{166}$, M. El Kacimi ${ }^{135 c}$, M. Ellert ${ }^{166}$, S. Elles ${ }^{5}$, F. Ellinghaus ${ }^{175}$, A. A. Elliot ${ }^{169}$, N. Ellis $^{30}$, J. Elmsheuser ${ }^{100}$, M. Elsing ${ }^{30}$, D. Emeliyanov ${ }^{131}$, Y. Enari ${ }^{155}$, O. C. Endner ${ }^{83}$, M. Endo ${ }^{118}$, J. Erdmann ${ }^{43}$, A. Ereditato ${ }^{17}$, G. Ernis ${ }^{175}$, J. Ernst ${ }^{2}$, M. Ernst ${ }^{25}$, S. Errede ${ }^{165}$, E. Ertel ${ }^{83}$, M. Escalier ${ }^{117}$, H. Esch $^{43}$, C. Escobar ${ }^{125}$, B. Esposito ${ }^{47}$, A. I. Etienvre ${ }^{136}$, E. Etzion ${ }^{153}$, H. Evans ${ }^{61}$, A. Ezhilov ${ }^{123}$, L. Fabbri ${ }^{20 a}, 20 b$, G. Facini ${ }^{31}$, R. M. Fakhrutdinov ${ }^{130}$, S. Falciano ${ }^{132 a}$, R. J. Falla ${ }^{78}$, J. Faltova ${ }^{129}$, Y. Fang ${ }^{33 a}$, M. Fanti ${ }^{91 a, 91 b}$, A. Farbin ${ }^{8}$, A. Farilla ${ }^{134 a}$, T. Farooque ${ }^{12}$, S. Farrell ${ }^{15}$, S. M. Farrington ${ }^{170}$, P. Farthouat ${ }^{30}$, F. Fassi ${ }^{135 e}$, P. Fassnacht ${ }^{30}$, D. Fassouliotis ${ }^{9}$, M. Faucci Giannelli ${ }^{77}$, A. Favareto ${ }^{50 a, 50 b}$, L. Fayard ${ }^{117}$, P. Federic ${ }^{144 a}$, O. L. Fedin ${ }^{123, m}$, W. Fedorko ${ }^{168}$, S. Feigl ${ }^{30}$, L. Feligioni ${ }^{85}$, C. Feng ${ }^{33 d}$, E. J. Feng ${ }^{6}$, H. Feng ${ }^{89}$, A. B. Fenyuk ${ }^{130}$, L. Feremenga ${ }^{8}$, P. Fernandez Martinez ${ }^{167}$, S. Fernandez Perez ${ }^{30}$, J. Ferrando ${ }^{53}$, A. Ferrari ${ }^{166}$, P. Ferrari ${ }^{107}$, R. Ferrari ${ }^{121 a}$, D. E. Ferreira de Lima ${ }^{53}$, A. Ferrer ${ }^{167}$, D. Ferrere ${ }^{49}$, C. Ferretti ${ }^{89}$, A. Ferretto Parodi ${ }^{50 a, 50 b}, \quad$ M. Fiascaris ${ }^{31}$, F. Fiedler ${ }^{83}$, A. Filipčič ${ }^{75}, \quad$ M. Filipuzzi ${ }^{42}$, F. Filthaut ${ }^{106}$, M. Fincke-Keeler ${ }^{169}$, K. D. Finelli ${ }^{150}$, M. C. N. Fiolhais ${ }^{126 a, 126 c}$, L. Fiorini ${ }^{167}$, A. Firan ${ }^{40}$, A. Fischer ${ }^{2}$,

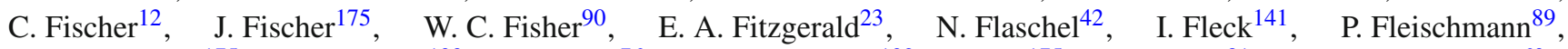
S. Fleischmann ${ }^{175}$, G. T. Fletcher ${ }^{139}$, G. Fletcher ${ }^{76}$, R. R. M. Fletcher ${ }^{122}$, T. Flick ${ }^{175}$, A. Floderus ${ }^{81}$, L. R. Flores Castillo ${ }^{60 a}$, M. J. Flowerdew ${ }^{101}$, A. Formica ${ }^{136}$, A. Forti ${ }^{84}$, D. Fournier ${ }^{117}$, H. Fox ${ }^{72}$, S. Fracchia ${ }^{12}$, P. Francavilla ${ }^{80}$, M. Franchini ${ }^{20 a}$,20b, D. Francis ${ }^{30}$, L. Franconi ${ }^{119}$, M. Franklin ${ }^{57}, \quad$ M. Frate ${ }^{163}$, M. Fraternali121a,121b, D. Freeborn ${ }^{78}, \quad$ S. T. French ${ }^{28}$, F. Friedrich ${ }^{44}$, D. Froidevaux ${ }^{30}$, J. A. Frost ${ }^{120}$, C. Fukunaga ${ }^{156}$, E. Fullana Torregrosa ${ }^{83}$, B. G. Fulsom ${ }^{143}$, T. Fusayasu ${ }^{102}$, J. Fuster ${ }^{167}$, C. Gabaldon ${ }^{55}$, O. Gabizon ${ }^{175}$, A. Gabrielli ${ }^{20 a, 20 b}$, A. Gabrielli1 ${ }^{132 a, 132 b}$, G. P. Gach ${ }^{38 a}$, S. Gadatsch ${ }^{30}$, S. Gadomski ${ }^{49}$, G. Gagliardi ${ }^{50 a, 50 b}$, P. Gagnon ${ }^{61}$, C. Galea ${ }^{106}$, B. Galhardo ${ }^{126 a, 126 c}$, E. J. Gallas ${ }^{120}$, B. J. Gallop ${ }^{131}$, P. Gallus ${ }^{128}$, G. Galster ${ }^{36}$, K. K. Gan ${ }^{111}$, J. Gao ${ }^{33 b, 85}$, Y. Gao ${ }^{46}$, Y. S. Gao ${ }^{143, e}$, F. M. Garay Walls ${ }^{46}$, F. Garberson ${ }^{176}$, C. García ${ }^{167}$, J. E. García Navarro ${ }^{167}$, M. Garcia-Sciveres ${ }^{15}$, R. W. Gardner ${ }^{31}$, N. Garelli ${ }^{143}$, V. Garonne ${ }^{119}$, C. Gatti ${ }^{47}$, A. Gaudiello ${ }^{50 a, 50 b}$, G. Gaudio ${ }^{121 a}$, B. Gaur ${ }^{141}$, L. Gauthier ${ }^{95}$, P. Gauzzi ${ }^{132 a, 132 b}$, I. L. Gavrilenko ${ }^{96}$, C. Gay ${ }^{168}$, G. Gaycken ${ }^{21}$, E. N. Gazis ${ }^{10}$, P. Ge ${ }^{33 d}$, Z. Gecse ${ }^{168}$, C. N. P. Gee ${ }^{131}$, Ch. Geich-Gimbel ${ }^{21}$, M. P. Geislerr ${ }^{58 a}$, C. Gemme ${ }^{50 a}$, M. H. Genest ${ }^{55}$, S. Gentile ${ }^{132 a, 132 b}$, M. George ${ }^{54}$, S. George $e^{77}$, D. Gerbaudo ${ }^{163}$, A. Gershon ${ }^{153}$, S. Ghasemi ${ }^{141}$, H. Ghazlane ${ }^{135 b}$, B. Giacobbe ${ }^{20 a}$, S. Giagu ${ }^{132 a, 132 b}$, V. Giangiobbe ${ }^{12}$, P. Giannetti ${ }^{124 a, 124 b}$, B. Gibbard ${ }^{25}$, S. M. Gibson ${ }^{77}$, M. Gilchriese ${ }^{15}$, T. P. S. Gillam ${ }^{28}$, D. Gillberg ${ }^{30}$, G. Gilles ${ }^{34}$, D. M. Gingrich ${ }^{3, d}$, N. Giokaris ${ }^{9}$, M. P. Giordani ${ }^{164 a, 164 c}$, F. M. Giorgi ${ }^{20 a}$, F. M. Giorgi ${ }^{16}$, P. F. Giraud ${ }^{136}$, P. Giromini ${ }^{47}$, D. Giugni ${ }^{91 a}$, C. Giuliani ${ }^{48}$, M. Giulini ${ }^{58 b}$, B. K. Gjelsten ${ }^{119}$, S. Gkaitatzis ${ }^{154}$, I. Gkialas ${ }^{154}$, E. L. Gkougkousis ${ }^{117}$, L. K. Gladilin ${ }^{99}$, C. Glasman ${ }^{82}$, J. Glatzer ${ }^{30}$, P. C. F. Glaysher ${ }^{46}$, A. Glazov ${ }^{42}$, M. Goblirsch-Kolb ${ }^{101}$, J. R. Goddard ${ }^{76}$, J. Godlewski ${ }^{39}$ ， S. Goldfarb ${ }^{89}$, T. Golling49, D. Golubkov ${ }^{130}$, A. Gomes ${ }^{126 a, 126 b, 126 d}$, R. Gonçalo ${ }^{126 a}$, J. Goncalves Pinto Firmino Da Costa ${ }^{136}$, L. Gonella ${ }^{21}$, S. González de la Hoz ${ }^{167}$, G. Gonzalez Parra ${ }^{12}$, S. Gonzalez-Sevilla ${ }^{49}$, L. Goossens ${ }^{30}$, P. A. Gorbounov ${ }^{97}$, H. A. Gordon ${ }^{25}$, I. Gorelov ${ }^{105}$, B. Gorini ${ }^{30}$, E. Gorinii ${ }^{73 a, 73 b}$, A. Gorišek ${ }^{75}$, E. Gornicki ${ }^{39}$, A. T. Goshaw ${ }^{45}$, C. Gössling ${ }^{43}$, M. I. Gostkin ${ }^{65}$, D. Goujdami ${ }^{135 c}$,

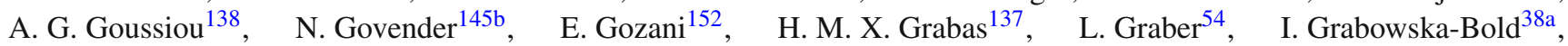
P. O. J. Gradin ${ }^{166}$, P. Grafström ${ }^{20 a, 20 b}$, K-J. Grahn ${ }^{42}$, J. Gramling ${ }^{49}$, E. Gramstad ${ }^{119}$, S. Grancagnolo ${ }^{16}$, V. Gratchev ${ }^{123}$, H. M. Gray ${ }^{30}$, E. Graziani134a, Z. D. Greenwood ${ }^{79, n}, \quad$ C. Grefe ${ }^{21}, \quad$ K. Gregersen ${ }^{78}$, I. M. Gregor ${ }^{42}, \quad$ P. Grenier ${ }^{143}$, J. Griffiths ${ }^{8}$, A. A. Grillo ${ }^{137}$, K. Grimm ${ }^{72}$, S. Grinstein ${ }^{12, o}$, Ph. Gris ${ }^{34}$, J.-F. Grivaz ${ }^{117}$, J. P. Grohs ${ }^{44}$, A. Grohsjean ${ }^{42}$, E. Gross ${ }^{172}$, J. Grosse-Knetter ${ }^{54}$, G. C. Grossi ${ }^{79}$, Z. J. Grout ${ }^{149}$, L. Guan ${ }^{89}$, J. Guenther ${ }^{128}$, F. Guescini ${ }^{49}$, D. Guest ${ }^{176}$, O. Gueta ${ }^{153}$, E. Guido ${ }^{50 a, 50 b}$, T. Guillemin ${ }^{117}, \quad$ S. Guindon ${ }^{2}, \quad$ U. Gul ${ }^{53}, \quad$ C. Gumpert ${ }^{44}, \quad$ J. Guo ${ }^{33 \mathrm{e}}$, Y. Guo ${ }^{33 \mathrm{~b}}$, S. Gupta ${ }^{120}$, G. Gustavino ${ }^{132 a, 132 b}$, P. Gutierrez ${ }^{113}$, N. G. Gutierrez Ortiz ${ }^{78}$, C. Gutschow ${ }^{44}$, C. Guyot ${ }^{136}$, C. Gwenlan ${ }^{120}$, C. B. Gwilliam ${ }^{74}$, A. Haas ${ }^{110}$, C. Haber ${ }^{15}$, H. K. Hadavand ${ }^{8}$, N. Haddad ${ }^{135 e}$, P. Haefner ${ }^{21}$, S. Hageböck ${ }^{21}$, Z. Hajduk ${ }^{39}$, H. Hakobyan ${ }^{177}$, M. Haleem ${ }^{42}$, J. Haley ${ }^{114}$, D. Hall ${ }^{120}$, G. Halladjian ${ }^{90}$, G. D. Hallewell ${ }^{85}$, K. Hamacher ${ }^{175}$, P. Hamal ${ }^{115}$,

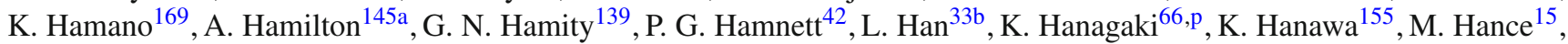
P. Hanke ${ }^{58 a}$, R. Hanna ${ }^{136}$, J. B. Hansen ${ }^{36}$, J. D. Hansen ${ }^{36}$, M. C. Hansen ${ }^{21}$, P. H. Hansen ${ }^{36}$, K. Hara ${ }^{160}$, A. S. Hard ${ }^{173}$, T. Harenberg ${ }^{175}$, F. Hariri ${ }^{117}$, S. Harkusha ${ }^{92}$, R. D. Harrington ${ }^{46}$, P. F. Harrison ${ }^{170}$, F. Hartjes ${ }^{107}$, M. Hasegawa ${ }^{67}$, 


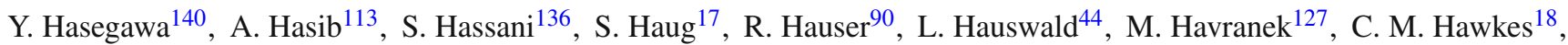
R. J. Hawkings ${ }^{30}$ ， A. D. Hawkins ${ }^{81}$ ， T. Hayashi ${ }^{160}$ ， D. Hayden ${ }^{90}$ ， C. P. Hays ${ }^{120}$ ， J. M. Hays ${ }^{76}$ ， H. S. Hayward ${ }^{74}$, S. J. Haywood ${ }^{131}$ ， S. J. Head ${ }^{18}$, T. Heck ${ }^{83}$, V. Hedberg ${ }^{81}$, L. Heelan ${ }^{8}$, S. Heim ${ }^{122}$, T. Heim ${ }^{175}$, B. Heinemann ${ }^{15}$, L. Heinrich ${ }^{110}$ ， J. Hejbal ${ }^{127}$ ， L. Helary ${ }^{22}$, S. Hellman ${ }^{146 a, 146 b}$ ， D. Hellmich ${ }^{21}$, C. Helsens ${ }^{12}$, J. Henderson ${ }^{120}$, R. C. W. Henderson ${ }^{72}$, Y. Heng ${ }^{173}$, C. Hengler ${ }^{42}$, S. Henkelmann ${ }^{168}$, A. Henrichs ${ }^{176}$, A. M. Henriques Correia ${ }^{30}$, S. Henrot-Versille ${ }^{117}$, G. H. Herbert ${ }^{16}$, Y. Hernández Jiménez ${ }^{167}$, R. Herrberg-Schubert ${ }^{16}$, G. Herten ${ }^{48}$, R. Hertenberger ${ }^{100}$, L. $\operatorname{Hervas}^{30}$ ， G. G. Hesketh ${ }^{78}$ ， N. P. Hessey ${ }^{107}$ ， J. W. Hetherly ${ }^{40}$ ，R. Hickling ${ }^{76}$ ，E. Higón-Rodriguez ${ }^{167}$, E. Hill ${ }^{169}$, J. C. Hill ${ }^{28}$, K. H. Hiller ${ }^{42}$, S. J. Hillier ${ }^{18}$, I. Hinchliffe ${ }^{15}$, E. Hines ${ }^{122}$, R. R. Hinman ${ }^{15}$, M. Hirose ${ }^{157}$, D. Hirschbuehl ${ }^{175}$, J. Hobbs ${ }^{148}$, N. Hod ${ }^{107}$, M. C. Hodgkinson ${ }^{139}$, P. Hodgson ${ }^{139}$, A. Hoecker ${ }^{30}$, M. R. Hoeferkamp ${ }^{105}$, F. Hoenig ${ }^{100}$, M. Hohlfeld ${ }^{83}$, D. Hohn ${ }^{21}$, T. R. Holmes ${ }^{15}$, M. Homann ${ }^{43}$, T. M. Hong ${ }^{125}$, L. Hooft van Huysduynen ${ }^{110}$, W. H. Hopkins ${ }^{116}$, Y. Horii ${ }^{103}$, A. J. Horton ${ }^{142}$, J-Y. Hostachy ${ }^{55}$, S. Hou ${ }^{151}$, A. Hoummada ${ }^{135 a}$, J. Howard ${ }^{120}$, J. Howarth ${ }^{42}$, M. Hrabovsky ${ }^{115}$, I. Hristova ${ }^{16}$, J. Hrivnac ${ }^{117}$, T. Hryn'ova ${ }^{5}$, A. Hrynevich ${ }^{93}$, C. Hsu ${ }^{145 c}$, P. J. Hsu ${ }^{151, q}$, S.-C. Hsu ${ }^{138}$, D. Hu ${ }^{35}$, Q. Hu ${ }^{33 b}$, X. Hu ${ }^{89}$, Y. Huang ${ }^{42}$, Z. Hubacek ${ }^{128}$, F. Hubaut ${ }^{85}$, F. Huegging ${ }^{21}$, T. B. Huffman ${ }^{120}$, E. W. Hughes ${ }^{35}$, G. Hughes ${ }^{72}$,

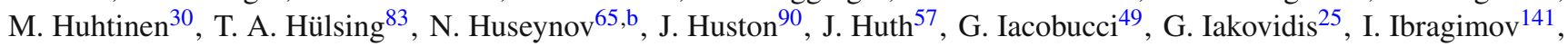
L. Iconomidou-Fayard ${ }^{117}$, E. Ideal ${ }^{176}$, Z. Idrissi ${ }^{135 e}$, P. Iengo ${ }^{30}$, O. Igonkina ${ }^{107}$, T. Iizawa ${ }^{171}$, Y. Ikegami ${ }^{66}$, K. Ikematsu $^{141}$,

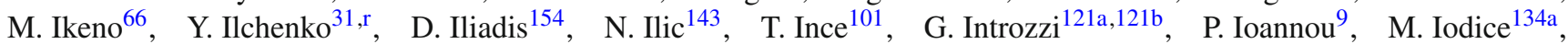
K. Iordanidou ${ }^{35}$, V. Ippolito ${ }^{57}$, A. Irles Quiles ${ }^{167}$, C. Isaksson ${ }^{166}$, M. Ishino ${ }^{68}$, M. Ishitsuka ${ }^{157}$, R. Ishmukhametov ${ }^{111}$, C. Issever ${ }^{120}$, S. Istin ${ }^{19 a}$, J. M. Iturbe Ponce ${ }^{84}$, R. Iuppa ${ }^{133 a, 133 b}$, J. Ivarsson ${ }^{81}$, W. Iwanski ${ }^{39}$, H. Iwasaki ${ }^{66}$, J. M. Izen ${ }^{41}$, V. Izzo ${ }^{104 a}$, S. Jabbar ${ }^{3}$, B. Jackson ${ }^{122}$, M. Jackson ${ }^{74}$, P. Jackson ${ }^{1}$, M. R. Jaekel ${ }^{30}$, V. Jain ${ }^{2}$, K. Jakobs ${ }^{48}$, S. Jakobsen ${ }^{30}$, T. Jakoubek ${ }^{127}$ ， J. Jakubek ${ }^{128}$ ， D. O. Jamin ${ }^{114}$ ， D. K. Jana ${ }^{79}$ ， E. Jansen ${ }^{78 ， \quad R . ~ J a n s k y ~}{ }^{62}$ ， J. Janssen ${ }^{21}$ ， M. Janus ${ }^{54}$, G. Jarlskog ${ }^{81}$ ， N. Javadov ${ }^{65, b}$ ， T. Javůrek ${ }^{48}$ ， L. Jeanty ${ }^{15}$ ，J. Jejelava ${ }^{51 a, s}$ ， G.-Y. Jeng ${ }^{150 ， \quad D . ~ J e n n e n s ~}{ }^{88}$ ， P. Jenni ${ }^{48, t}$, J. Jentzsch ${ }^{43}$ ， C. Jeske ${ }^{170}$ ， S. Jézéquel ${ }^{5}$, H. Ji ${ }^{173}$ ， J. Jia ${ }^{148}$ ， Y. Jiang ${ }^{33 b}$ ， S. Jiggins ${ }^{78}$, J. Jimenez Pena ${ }^{167}$, S. Jin ${ }^{33 a}$, A. Jinaru ${ }^{26 a}$, O. Jinnouchi ${ }^{157}$, M. D. Joergensen ${ }^{36}$, P. Johansson ${ }^{139}$, K. A. Johns ${ }^{7}$, K. Jon-And ${ }^{146 a, 146 b}$, G. Jones ${ }^{170}$, R. W. L. Jones ${ }^{72}$, T. J. Jones ${ }^{74}$, J. Jongmanns ${ }^{58 a}$, P. M. Jorge ${ }^{126 a, 126 b}$, K. D. Joshi ${ }^{84}$, J. Jovicevic ${ }^{159 a}$, X. Ju ${ }^{173}$, C. A. Jung ${ }^{43}$, P. Jussel ${ }^{62}$, A. Juste Rozas ${ }^{12, o}$, M. Kaci ${ }^{167}$, A. Kaczmarska ${ }^{39}$, M. Kado ${ }^{117}$, H. Kagan ${ }^{111}$, M. Kagan ${ }^{143}$, S. J. Kahn ${ }^{85}$, E. Kajomovitz ${ }^{45}$, C. W. Kalderon ${ }^{120}$, S. Kama ${ }^{40}$, A. Kamenshchikov ${ }^{130}$, N. Kanaya ${ }^{155}$, S. Kaneti ${ }^{28}$, V. A. Kantserov ${ }^{98}$, J. Kanzaki ${ }^{66}$, B. Kaplan ${ }^{110}$, L. S. Kaplan ${ }^{173}$, A. Kapliy ${ }^{31}$, D. Kar ${ }^{145 c}$, K. Karakostas ${ }^{10}$, A. Karamaoun ${ }^{3}$, N. Karastathis ${ }^{10,107}$, M. J. Kareem ${ }^{54}$, E. Karentzos ${ }^{10}$, M. Karnevskiy ${ }^{83}$, S. N. Karpov ${ }^{65}$, Z. M. Karpova ${ }^{65}$, K. Karthik ${ }^{110}$, V. Kartvelishvili ${ }^{72}$, A. N. Karyukhin ${ }^{130}$, L. Kashif ${ }^{173}$, R. D. Kass ${ }^{111}$, A. Kastanas ${ }^{14}$, Y. Kataoka ${ }^{155}$, C. Kato ${ }^{155}$, A. Katre ${ }^{49}$, J. Katzy ${ }^{42}$, K. Kawagoe ${ }^{70}$, T. Kawamoto ${ }^{155}$, G. Kawamura ${ }^{54}$, S. Kazama ${ }^{155}$, V. F. Kazanin ${ }^{109, c}$, R. Keeler ${ }^{169}$, R. Kehoe ${ }^{40}$, J. S. Keller ${ }^{42}$, J. J. Kempster ${ }^{77}$, H. Keoshkerian ${ }^{84}$, O. Kepka ${ }^{127}$, B. P. Kerševan ${ }^{75}$, S. Kersten ${ }^{175}$, R. A. Keyes ${ }^{87}$, F. Khalil-zada ${ }^{11}$, $\begin{array}{lll}\text { H. Khandanyan } & 146 \mathrm{a}, 146 \mathrm{~b} & \text { A. Khanov } \\ & & \end{array}$ J. Khubua ${ }^{51 b, u}$, S. Kido ${ }^{67}$, H. Y. Kim ${ }^{8}$, S. H. Kim ${ }^{160}$, Y. K. Kim ${ }^{31}$, N. Kimura ${ }^{154}$, O. M. Kind ${ }^{16}$, B. T. King ${ }^{74}$, M. King ${ }^{167}$, S. B. King ${ }^{168}$, J. Kirk ${ }^{131}$, A. E. Kiryunin ${ }^{101}$, T. Kishimoto ${ }^{67}$, D. Kisielewska ${ }^{38 a}$, F. Kiss ${ }^{48}$, K. Kiuchi ${ }^{160}$, O. Kivernyk ${ }^{136}$, E. Kladiva ${ }^{144 b}$, M. H. Klein ${ }^{35}$, M. Klein ${ }^{74}$, U. Klein ${ }^{74}$, K. Kleinknecht ${ }^{83}$, P. Klimek ${ }^{146 a, 146 b}$, A. Klimentov ${ }^{25}$, R. Klingenberg ${ }^{43}$, J. A. Klinger ${ }^{139}$, T. Klioutchnikova ${ }^{30}$, E.-E. Kluge ${ }^{58 a}$, P. Kluit ${ }^{107}$ ， S. Kluth ${ }^{101}$, J. Knapik ${ }^{39}$, E. Kneringer ${ }^{62}$, E. B. F. G. Knoops ${ }^{85}$, A. Knue ${ }^{53}$, A. Kobayashi ${ }^{155}$, D. Kobayashi ${ }^{157}$, T. Kobayashi ${ }^{155}$, M. Kobel ${ }^{44}$, M. Kocian ${ }^{143}$, P. Kodys ${ }^{129}$, T. Koffas ${ }^{29}$, E. Koffeman ${ }^{107}$, L. A. Kogan ${ }^{120}$, S. Kohlmann ${ }^{175}$, Z. Kohout ${ }^{128}$, T. Kohriki ${ }^{66}$, T. Koi ${ }^{143}$, H. Kolanoski ${ }^{16}$, I. Koletsou ${ }^{5}$, A. A. Komar ${ }^{96, *}$, Y. Komori ${ }^{155}$, T. Kondo ${ }^{66}$, N. Kondrashova ${ }^{42}$, K. Köneke ${ }^{48}$, A. C. König ${ }^{106}$, T. Kono ${ }^{66}$, R. Konoplich ${ }^{110, v}$, N. Konstantinidis ${ }^{78}$, R. Kopeliansky ${ }^{152}$, S. Koperny ${ }^{38 a}$, L. Köpke ${ }^{83}$, A. K. Kopp ${ }^{48}$, K. Korcyl ${ }^{39}$, K. Kordas ${ }^{154}$, A. Korn ${ }^{78}$, A. A. Korol ${ }^{109, c}$, I. Korolkov 12 , E. V. Korolkova ${ }^{139}$, O. Kortner ${ }^{101}$, S. Kortner ${ }^{101}$, T. Kosek ${ }^{129}$, V. V. Kostyukhin ${ }^{21}$, V. M. Kotov ${ }^{65}$, A. Kotwal ${ }^{45}$, A. Kourkoumeli-Charalampidi ${ }^{154}$, C. Kourkoumelis ${ }^{9}$, V. Kouskoura ${ }^{25}$, A. Koutsman ${ }^{159 a}$, R. Kowalewski ${ }^{169}$, T. Z. Kowalski ${ }^{38 a}$, W. Kozanecki ${ }^{136}$, A. S. Kozhin ${ }^{130}$, V. A. Kramarenko ${ }^{99}$, G. Kramberger ${ }^{75}$, D. Krasnopevtsev ${ }^{98}$, M. W. Krasny ${ }^{80}$, A. Krasznahorkay ${ }^{30}$, J. K. Kraus ${ }^{21}$, A. Kravchenko ${ }^{25}$, S. Kreiss ${ }^{110}$, M. Kretz ${ }^{58 c}$, J. Kretzschmar ${ }^{74}$, K. Kreutzfeldt ${ }^{52}$, P. Krieger ${ }^{158}$, K. Krizka ${ }^{31}$, K. Kroeninger ${ }^{43}$, H. Kroha ${ }^{101}$, J. Kroll ${ }^{122}$, J. Kroseberg ${ }^{21}$, J. Krstic ${ }^{13}$, U. Kruchonak ${ }^{65}$, H. Krüger ${ }^{21}$, N. Krumnack ${ }^{64}$, A. Kruse ${ }^{173}$, M. C. $\mathrm{Kruse}^{45}$, M. Kruskal ${ }^{22}$, T. Kubota ${ }^{88}$, H. Kucuk ${ }^{78}$, S. Kuday ${ }^{4 b}$, S. Kuehn ${ }^{48}$, A. Kugel ${ }^{58 c}$, F. Kuger ${ }^{174}$, A. Kuhl ${ }^{137}$, T. Kuhl ${ }^{42}$, V. Kukhtin ${ }^{65}$, R. Kukla ${ }^{136}$, Y. Kulchitsky ${ }^{92}$, S. Kuleshov ${ }^{32 b}$, M. Kuna ${ }^{132 a, 132 b}$, T. Kunigo ${ }^{68}$, A. Kupco ${ }^{127}$, H. Kurashige ${ }^{67}$, Y. A. Kurochkin ${ }^{92}$, V. Kus ${ }^{127}$, E. S. Kuwertz ${ }^{169}$, M. Kuze ${ }^{157}$, J. Kvita ${ }^{115}$, T. Kwan ${ }^{169}$, D. Kyriazopoulos ${ }^{139}$, A. La Rosa ${ }^{137}$, J. L. La Rosa Navarro ${ }^{24 d}$, L. La Rotonda ${ }^{37 a, 37 b}$, C. Lacasta ${ }^{167}$, F. Lacava ${ }^{132 a, 132 b}$,

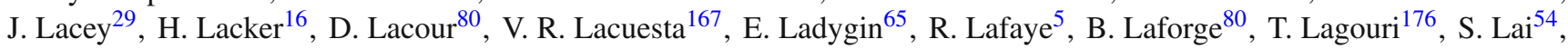
L. Lambourne ${ }^{78}$, S. Lammers ${ }^{61}$, C. L. Lampen ${ }^{7}$, W. Lampl ${ }^{7}$, E. Lançon ${ }^{136}$, U. Landgraf ${ }^{48}$, M. P. J. Landon ${ }^{76}$, V. S. Lang ${ }^{58 a}$, J. C. Lange ${ }^{12}$, A. J. Lankford ${ }^{163}$, F. Lanni ${ }^{25}$, K. Lantzsch ${ }^{21}$, A. Lanza ${ }^{121 a}$, S. Laplace ${ }^{80}$, C. Lapoire ${ }^{30}$, J. F. Laporte ${ }^{136}$, 


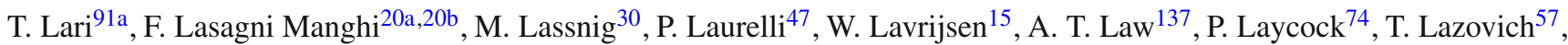
O. Le Dortz ${ }^{80}$, E. Le Guirriec ${ }^{85}$, E. Le Menedeu ${ }^{12}$, M. LeBlanc ${ }^{169}$, T. LeCompte ${ }^{6}$, F. Ledroit-Guillon ${ }^{55}$, C. A. Lee ${ }^{145 b}$, S. C. Lee ${ }^{151}$, L. Lee ${ }^{1}$, G. Lefebvre ${ }^{80}$, M. Lefebvre ${ }^{169}$, F. Legger ${ }^{100}$, C. Leggett ${ }^{15}$, A. Lehan ${ }^{74}$, G. Lehmann Miotto ${ }^{30}$, X. Lei ${ }^{7}$, W. A. Leight ${ }^{29}$, A. Leisos ${ }^{154, w}$, A. G. Leister ${ }^{176}$, M. A. L. Leite ${ }^{24 d}$, R. Leitner ${ }^{129}$, D. Lellouch ${ }^{172}$, B. Lemmer ${ }^{54}$, K. J. C. Leney ${ }^{78}$, T. Lenz ${ }^{21}$, B. Lenzi ${ }^{30}$, R. Leone ${ }^{7}$, S. Leone ${ }^{124 a, 124 b}$, C. Leonidopoulos ${ }^{46}$, S. Leontsinis ${ }^{10}$, C. Leroy ${ }^{95}$, C. G. Lester ${ }^{28}$, M. Levchenko ${ }^{123}$, J. Levêque ${ }^{5}$, D. Levin $^{89}$, L. J. Levinson ${ }^{172}$, M. Levy ${ }^{18}$, A. Lewis ${ }^{120}$, A. M. Leyko ${ }^{21}$,

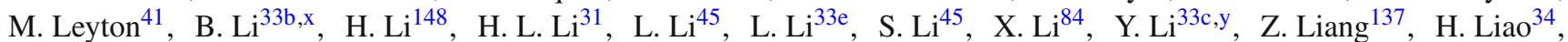
B. Liberti ${ }^{133 a}$, A. Liblong ${ }^{158}$, P. Lichard ${ }^{30}, \quad$ K. Lie $^{165}$, J. Liebal ${ }^{21}$ ， W. Liebig ${ }^{14}, \quad$ C. Limbach ${ }^{21}$ A. Limosani ${ }^{150}$, S. C. $\operatorname{Lin}^{151, \mathrm{z}}$, T. H. $\operatorname{Lin}^{83}$, F. Linde ${ }^{107}$, B. E. Lindquist ${ }^{148}$, J. T. Linnemann ${ }^{90}$, E. Lipeles ${ }^{122}$, A. Lipniacka ${ }^{14}$, M. Lisovyi ${ }^{58 b}$, T. M. Liss ${ }^{165}$ ，D. Lissauer ${ }^{25}$ ，A. Lister ${ }^{168}$ ，A. M. Litke ${ }^{137}$ ，B. Liu ${ }^{151, a a ， D . ~ L i u}{ }^{151} ，$ H. Liu ${ }^{89}$ ，J. Liu ${ }^{85}$ ，J. B. Liu ${ }^{33 b}$,

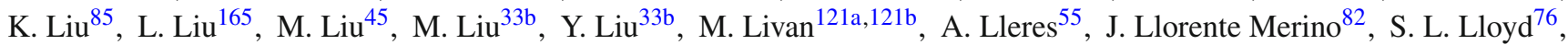
$\begin{array}{llll}\text { F. Lo Sterzo } & 151 & \text { E. Lobodzinska }\end{array}{ }^{42}$, P. Loch ${ }^{7}$, W. S. Lockman ${ }^{137}$ ， F. K. Loebinger ${ }^{84}$, A. E. Loevschall-Jensen ${ }^{36}$, A. Loginov ${ }^{176}$, T. Lohse ${ }^{16}$, K. Lohwasser ${ }^{42}$, M. Lokajicek ${ }^{127}$, B. A. Long ${ }^{22}$, J. D. Long ${ }^{89}$, R. E. Long ${ }^{72}$, K. A. Looper ${ }^{111}$, $\begin{array}{lll}\text { L. Lopes } & \text { 126a } & \text { D. Lopez Mateos }\end{array}$

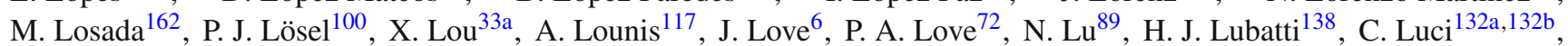
A. Lucotte ${ }^{55}$, F. Luehring ${ }^{61}$, W. Lukas ${ }^{62}$, L. Luminari132a, O. Lundberg ${ }^{146 a, 146 b}, \quad$ B. Lund-Jensen ${ }^{147}, \quad$ D. Lynn ${ }^{25}$, R. Lysak ${ }^{127}$, E. Lytken ${ }^{81}$, H. Ma ${ }^{25}$, L. L. Ma ${ }^{33 d}$, G. Maccarrone ${ }^{47}$, A. Macchiolo ${ }^{101}$, C. M. Macdonald ${ }^{139}$, B. Maček ${ }^{75}$, J. Machado Miguens ${ }^{122,126 b}$, D. Macina ${ }^{30}$, D. Madaffari ${ }^{85}$, R. Madar ${ }^{34}$, H. J. Maddocks ${ }^{72}$, W. F. Mader ${ }^{44}$, A. Madsen ${ }^{166}$, J. Maeda $^{67}$, S. Maeland ${ }^{14}$, T. Maeno ${ }^{25}$, A. Maevskiy ${ }^{99}$, E. Magradze ${ }^{54}$, K. Mahboubi ${ }^{48}$, J. Mahlstedt ${ }^{107}$, C. Maiani ${ }^{136}$, C. Maidantchik ${ }^{24 a}$, A. A. Maier ${ }^{101}$, T. Maier ${ }^{100}$, A. Maio ${ }^{126 a, 126 b, 126 d}$, S. Majewski ${ }^{116}$, Y. Makida ${ }^{66}$, N. Makovec ${ }^{117}$, B. Malaescu ${ }^{80}$, Pa. Malecki ${ }^{39}$, V. P. Maleev ${ }^{123}$, F. Malek ${ }^{55}$, U. Mallik ${ }^{63}$, D. Malon ${ }^{6}$, C. Malone ${ }^{143}$, S. Maltezos ${ }^{10}$, V. M. Malyshev ${ }^{109}$, S. Malyukov ${ }^{30}$, J. Mamuzic ${ }^{42}, \quad$ G. Mancini ${ }^{47}, \quad$ B. Mandelli ${ }^{30}$, L. Mandelli ${ }^{91 a}$, I. Mandić ${ }^{75}$, R. Mandrysch ${ }^{63}$, J. Maneira 126a,126b, A. Manfredini ${ }^{101}$, L. Manhaes de Andrade Filho ${ }^{24 b}$, J. Manjarres Ramos ${ }^{159 b}$, A. Mann ${ }^{100}$, A. Manousakis-Katsikakis ${ }^{9}$, B. Mansoulie ${ }^{136}$, R. Mantifel ${ }^{87}$, M. Mantoani ${ }^{54}$, L. Mapelli ${ }^{30}$, L. March ${ }^{145 c}$, G. Marchiori ${ }^{80}$, M. Marcisovsky ${ }^{127}$, C. P. Marino ${ }^{169}$, M. Marjanovic ${ }^{13}$, D. E. Marley ${ }^{89}$, F. Marroquim² ${ }^{24 a}$, S. P. Marsden ${ }^{84}$, Z. Marshall ${ }^{15}$, L. F. Marti ${ }^{17}$, S. Marti-Garcia ${ }^{167}$, B. Martin ${ }^{90}$, T. A. Martin ${ }^{170}$, V. J. Martin ${ }^{46}$, B. Martin dit Latour ${ }^{14}$, M. Martinez ${ }^{12, o}$, S. Martin-Haugh ${ }^{131}$, V. S. Martoiu ${ }^{26 a}$, A. C. Martyniuk ${ }^{78}$, M. Marx ${ }^{138}$, F. Marzano ${ }^{132 a}$, A. Marzin ${ }^{30}$, L. Masetti ${ }^{83}$, T. Mashimo ${ }^{155}$, R. Mashinistov ${ }^{96}$, J. Masik ${ }^{84}$, A. L. Maslennikov ${ }^{109, c}$, I. Massa ${ }^{20 a, 20 b}$, L. Massa ${ }^{20 a, 20 b}$, P. Mastrandrea ${ }^{148}$, A. Mastroberardino ${ }^{37 a, 37 b}, \quad$ T. Masubuchi ${ }^{155}, \quad$ P. Mättig ${ }^{175}$ ， J. Mattmann ${ }^{83}$, J. Maurer ${ }^{26 a}$, S. J. Maxfield ${ }^{74}$ ， D. A. Maximov ${ }^{109, c}$, R. Mazini ${ }^{151}$ ， S. M. Mazza ${ }^{91 a, 91 b}$ ， L. Mazzaferro ${ }^{133 a, 133 b}$, G. Mc Goldrick ${ }^{158}$,

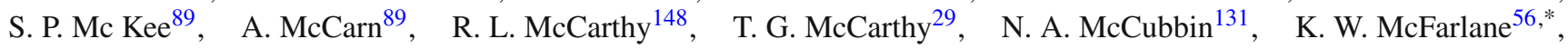

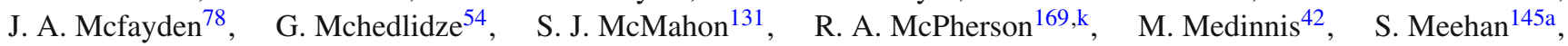
S. Mehlhase ${ }^{100}$, A. Mehta ${ }^{74}$, K. Meier ${ }^{58 a}$, C. Meineck ${ }^{100}$, B. Meirose ${ }^{41}$ ， B. R. Mellado Garcia ${ }^{145 c}$, F. Meloni ${ }^{17}$, A. Mengarelli ${ }^{20 a, 20 b}$, S. Menke ${ }^{101}$, E. Meoni ${ }^{161}$, K. M. Mercurio ${ }^{57}$, S. Mergelmeyer ${ }^{21}$, P. Mermod ${ }^{49}$, L. Merola ${ }^{104 a, 104 b}$, C. Meroni ${ }^{11 a}$, F. S. Merritt ${ }^{31}$, A. Messina ${ }^{132 a, 132 b}$, J. Metcalfe ${ }^{25}$, A. S. Mete ${ }^{163}$, C. Meyer ${ }^{83}$, C. Meyer ${ }^{122}$, J-P. Meyer ${ }^{136}$, J. Meyer $^{107}$, H. Meyer Zu Theenhausen ${ }^{58 a}$, R. P. Middleton ${ }^{131}$, S. Miglioranzi ${ }^{164 a, 164 c}$, L. Mijović21 ${ }^{21}$ G. Mikenberg ${ }^{172}$, M. Mikestikova ${ }^{127}$, M. Mikuž ${ }^{75}$, M. Milesi ${ }^{88}$, A. Milic ${ }^{30}$, D. W. Miller ${ }^{31}$, C. Mills ${ }^{46}$, A. Milov ${ }^{172}$, D. A. Milstead ${ }^{146 a, 146 b}$, A. A. Minaenko ${ }^{130}$, Y. Minami ${ }^{155}$, I. A. Minashvili ${ }^{65}$, A. I. Mincer ${ }^{110}$, B. $\operatorname{Mindur}^{38 a}$, M. Mineev ${ }^{65}$, Y. Ming ${ }^{173}$, L. M. Mir ${ }^{12}$, T. Mitani ${ }^{171}$, J. Mitrevski ${ }^{100}$, V. A. Mitsou ${ }^{167}$, A. Miucci ${ }^{49}$, P. S. Miyagawa ${ }^{139}$ ， J. U. Mjörnmark ${ }^{81}$, T. Moa ${ }^{146 a, 146 b}$, K. Mochizuki ${ }^{85}$, S. Mohapatra ${ }^{35}$, W. Mohr ${ }^{48}$, S. Molander ${ }^{146 a, 146 b}$, R. Moles-Valls ${ }^{21}$, R. Monden ${ }^{68}$, K. Mönig ${ }^{42}$, C. Monini ${ }^{55}$, J. Monk ${ }^{36}$, E. Monnier ${ }^{85}$, J. Montejo Berlingen ${ }^{12}$, F. Monticelli1 ${ }^{71}$, S. Monzani ${ }^{132 a, 132 b}$, R. W. Moore ${ }^{3}$, N. Morange ${ }^{117}$ ， D. Moreno ${ }^{162}$, M. Moreno Llácer ${ }^{54}$, P. Morettini ${ }^{50 a}$, D. Mori ${ }^{142}, \quad$ M. Morii ${ }^{57}, \quad$ M. Morinaga ${ }^{155}$, V. Morisbak ${ }^{119}$, S. Moritz ${ }^{83}$, A. K. Morley ${ }^{150}$, G. Mornacchi ${ }^{30}$, J. D. Morris ${ }^{76}$, S. S. Mortensen ${ }^{36}$, A. Morton ${ }^{53}$, L. Morvaj ${ }^{103}$ ， M. Mosidze ${ }^{51 b}$ ， J. Moss ${ }^{143}$ ， K. Motohashi ${ }^{157}$ ， R. Mount ${ }^{143}$ ， E. Mountricha ${ }^{25}$ ， S. V. Mouraviev ${ }^{96, *}$,

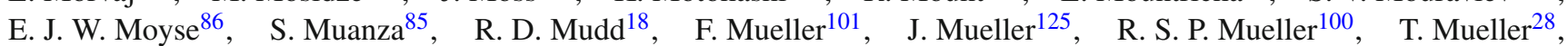
D. Muenstermann ${ }^{49}$, P. Mullen ${ }^{53}$, G. A. Mullier ${ }^{17}$, J. A. Murillo Quijada ${ }^{18}$, W. J. Murray ${ }^{170,131}$, H. Musheghyan ${ }^{54}$, E. Musto ${ }^{152}$, A. G. Myagkov ${ }^{130, a b}$, M. Myska ${ }^{128}$ ， B. P. Nachman ${ }^{143}$, O. Nackenhorst ${ }^{54}$ ， J. Nadal ${ }^{54}$, K. Nagai ${ }^{120}$, R. Nagai ${ }^{157}$ ， Y. Nagai ${ }^{85}$ ， K. Nagano ${ }^{66}$ ， A. Nagarkar ${ }^{111}$ ， Y. Nagasaka ${ }^{59}, \quad$ K. Nagata ${ }^{160} ，$ M. Nagel ${ }^{101}, \quad$ E. Nagy ${ }^{85}$, A. M. Nairz ${ }^{30}$, Y. Nakahama ${ }^{30}$, K. Nakamura ${ }^{66}$, T. Nakamura ${ }^{155}$, I. Nakano ${ }^{112}$, H. Namasivayam ${ }^{41}$, R. F. Naranjo Garcia ${ }^{42}$, R. Narayan ${ }^{31}$ ， D. I. Narrias Villar ${ }^{58 a}$ ， T. Naumann ${ }^{42}$ ， G. Navarro ${ }^{162}$ ， R. Nayyar ${ }^{7}$, H. A. Neal ${ }^{89}$ ， P. Yu. Nechaeva ${ }^{96}$, T. J. Neep ${ }^{84}$, P. D. Nef ${ }^{143}$, A. Negri ${ }^{121 a, 121 b}$, M. Negrini ${ }^{20 a}$, S. Nektarijevic ${ }^{106}$, C. Nellist ${ }^{117}$, A. Nelson ${ }^{163}$, S. Nemecek ${ }^{127}$, P. Nemethy ${ }^{110}$, A. A. Nepomuceno ${ }^{24 a}$, M. Nessi ${ }^{30, a c}$, M. S. Neubauer ${ }^{165}$, M. Neumann ${ }^{175}$, R. M. Neves ${ }^{110}$, P. Nevski ${ }^{25}$, P. R. Newman ${ }^{18}$, D. H. Nguyen ${ }^{6}$, R. B. Nickerson ${ }^{120}$, R. Nicolaidou ${ }^{136}$, B. Nicquevert ${ }^{30}$, J. Nielsen ${ }^{137}$, N. Nikiforou ${ }^{35}$, 
A. Nikiforov ${ }^{16}$, V. Nikolaenko ${ }^{130, a b}$, I. Nikolic-Audit ${ }^{80}$, K. Nikolopoulos ${ }^{18}$, J. K. Nilsen ${ }^{119}$, P. Nilsson ${ }^{25}$, Y. Ninomiya ${ }^{155}$, A. Nisati ${ }^{132 a}$, R. Nisius ${ }^{101}$, T. Nobe ${ }^{155}$, M. Nomachi ${ }^{118}$, I. Nomidis ${ }^{29}$, T. Nooney ${ }^{76}$, S. Norberg ${ }^{113}$, M. Nordberg ${ }^{30}$, O. Novgorodova ${ }^{44}$ ，S. Nowak ${ }^{101}$ ，M. Nozaki ${ }^{66}$ ，L. Nozka ${ }^{115}$ ，K. Ntekas ${ }^{10}$ ， G. Nunes Hanninger ${ }^{88}$, T. Nunnemann ${ }^{100}$, E. Nurse ${ }^{78}$, F. Nuti ${ }^{88}$, B. J. O’Brien ${ }^{46}$, F. O’grady ${ }^{7}$, D. C. O’Neil ${ }^{142}$, V. O’Shea ${ }^{53}$, F. G. Oakham ${ }^{29, d}$, H. Oberlack ${ }^{101}$, T. Obermann ${ }^{21}$, J. Ocariz ${ }^{80}$, A. Ochi ${ }^{67}$, I. Ochoa ${ }^{78}$, J. P. Ochoa-Ricoux ${ }^{32 a}$, S. Oda ${ }^{70}$, S. Odaka ${ }^{66}$, H. Ogren ${ }^{61}$, A. Oh ${ }^{84}$, S. H. $\mathrm{Oh}^{45}$, C. C. $\mathrm{Ohm}^{15}$, H. Ohman ${ }^{166}$, H. Oide ${ }^{30}$, W. Okamura ${ }^{118}$, H. Okawa ${ }^{160}$, Y. Okumura ${ }^{31}$, T. Okuyama ${ }^{66}$, A. Olariu ${ }^{26 a}$, S. A. Olivares Pino ${ }^{46}$, D. Oliveira Damazio ${ }^{25}$, E. Oliver Garcia ${ }^{167}$, A. Olszewski ${ }^{39}$ ， J. Olszowska ${ }^{39}$, A. Onofre ${ }^{126 a, 126 e}$, K. Onogi ${ }^{103}$ ，P. U. E. Onyisi ${ }^{31, r}$ ， C. J. Oram ${ }^{159 a}$ ， M. J. Oreglia ${ }^{31}$ ，Y. Oren ${ }^{153}$ ，D. Orestano ${ }^{134 a, 134 b}$, N. Orlando ${ }^{154}$, C. Oropeza Barrera ${ }^{53}$, R. S. Orr ${ }^{158}$, B. Osculati ${ }^{50 a, 50 b}$, R. Ospanov ${ }^{84}$, G. Otero y Garzon ${ }^{27}$, H. Otono ${ }^{70}$, M. Ouchrif ${ }^{135 d}$, F. Ould-Saada ${ }^{119}$, A. Ouraou ${ }^{136}$, K. P. Oussoren ${ }^{107}$, Q. Ouyang ${ }^{33 a}$, A. Ovcharova ${ }^{15}$, M. Owen ${ }^{53}$, R. E. Owen ${ }^{18}$, V. E. Ozcan ${ }^{19 a}$, N. Ozturk ${ }^{8}$, K. Pachal ${ }^{142}$, A. Pacheco Pages ${ }^{12}$, C. Padilla Aranda ${ }^{12}$, M. Pagáčováá ${ }^{48}$, S. Pagan Griso ${ }^{15}$, E. Paganis ${ }^{139}$, F. Paige ${ }^{25}$, P. Pais ${ }^{86}$, K. Pajchel ${ }^{119}$, G. Palacino ${ }^{159 b}$, S. Palestini ${ }^{30}$, M. Palka ${ }^{38 b}$,

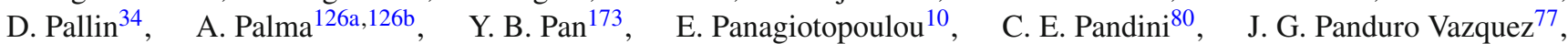
P. Pani ${ }^{146 a, 146 b}$, S. Panitkin ${ }^{25}$, D. Pantea ${ }^{26 a}$, L. Paolozzi ${ }^{49}$, Th. D. Papadopoulou ${ }^{10}$, K. Papageorgiou ${ }^{154}$, A. Paramonov ${ }^{6}$,

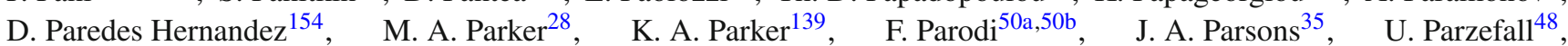
E. Pasqualucci132a, S. Passaggio ${ }^{50 a}$ ， F. Pastore ${ }^{134 a, 134 b, *}$, Fr. Pastore ${ }^{77}$ ， G. Pásztor ${ }^{29}$, S. Pataraia ${ }^{175}$, N. D. Patel ${ }^{150}$, J. R. Pater $^{84}$ ， T. Pauly ${ }^{30}$ ， J. Pearce ${ }^{169}$ ， B. Pearson ${ }^{113}$ ， L. E. Pedersen ${ }^{36}, \quad$ M. Pedersen ${ }^{119} ，$ S. Pedraza Lopez ${ }^{167}$, R. Pedro ${ }^{126 a, 126 b}$, S. V. Peleganchuk ${ }^{109, c}$, D. Pelikan ${ }^{166}$, O. Penc ${ }^{127}$, C. Peng ${ }^{33 a}$, H. Peng ${ }^{33 b}$, B. Penning ${ }^{31}$, J. Penwell ${ }^{61}$, D. V. Perepelitsa ${ }^{25}$, E. Perez Codina ${ }^{159 a}$, M. T. Pérez García-Estañ ${ }^{167}$, L. Perini ${ }^{91 a, 91 b}$, H. Pernegger ${ }^{30}$, S. Perrella ${ }^{104 a, 104 b}$, R. Peschke $e^{42}$, V. D. Peshekhonov ${ }^{65}$, K. Peters ${ }^{30}$, R. F. Y. Peters ${ }^{84}$, B. A. Petersen ${ }^{30}$, T. C. Petersen $^{36}$, E. Petit ${ }^{42}$, A. Petridis ${ }^{1}$, C. Petridou ${ }^{154}$, P. Petroff ${ }^{117}$, E. Petrolo ${ }^{132 a}$, F. Petrucci ${ }^{134 a, 134 b}$ ， N. E. Pettersson ${ }^{157}$, R. Pezoa ${ }^{32 b}$, P. W. Phillips ${ }^{131}$, G. Piacquadio ${ }^{143}$, E. Pianori ${ }^{170}$, A. Picazio ${ }^{49}$, E. Piccaro ${ }^{76}$, M. Piccinini ${ }^{20 a, 20 b}$, M. A. Pickering ${ }^{120}$, R. Piegaia ${ }^{27}$, D. T. Pignotti ${ }^{111}$, J. E. Pilcher ${ }^{31}$, A. D. Pilkington ${ }^{84}, \quad$ J. Pina ${ }^{126 a, 126 b, 126 d}, \quad$ M. Pinamonti ${ }^{164 a, 164 c, a d}$, J. L. Pinfold ${ }^{3}$, A. Pingel ${ }^{36}$, S. Pires ${ }^{80}, \quad$ H. Pirumov ${ }^{42}$, M. Pitt ${ }^{172}, \quad$ C. Pizio ${ }^{91 a}, 91 b, \quad$ L. Plazak ${ }^{144 a}, \quad$ M.-A. Pleier ${ }^{25}$, V. Pleskot ${ }^{129}$, E. Plotnikova ${ }^{65}$, P. Plucinski 146a,146b , D. Pluth ${ }^{64}$, R. Poettgen ${ }^{146 a, 146 b}$ ， L. Poggioli117, D. Pohl ${ }^{21}$, G. Polesello ${ }^{121 a}$, A. Poley ${ }^{42}$, A. Policicchio ${ }^{37 a, 37 b}$, R. Polifka ${ }^{158}$, A. Polini ${ }^{20 a}$, C. S. Pollard ${ }^{53}$, V. Polychronakos ${ }^{25}$, K. Pommès ${ }^{30}$, L. Pontecorvo ${ }^{132 a}$, B. G. Pope ${ }^{90}$, G. A. Popeneciu ${ }^{26 b}$, D. S. Popovic ${ }^{13}$, A. Poppleton ${ }^{30}$, S. Pospisil ${ }^{128}$, K. Potamianos ${ }^{15}$, I. N. Potrap ${ }^{65}$, C. J. Potter ${ }^{149}$, C. T. Potter ${ }^{16}$, G. Poulard ${ }^{30}$, J. Poveda ${ }^{30}$, V. Pozdnyakov ${ }^{65}$, P. Pralavorio ${ }^{85}$, A. Pranko ${ }^{15}$,

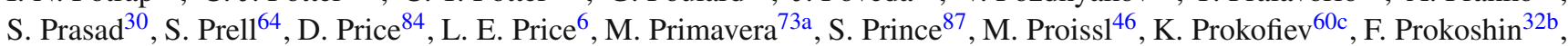
E. Protopapadaki ${ }^{136}$, S. Protopopescu ${ }^{25}$, J. Proudfoot ${ }^{6}$, M. Przybycien $^{38 a}$, E. Ptacek ${ }^{116}$, D. Puddu ${ }^{134 a, 134 b}$, E. Pueschel ${ }^{86}$, D. Puldon ${ }^{148}$, M. Purohit ${ }^{25}$,ae, P. Puzo ${ }^{117}$, J. Qian ${ }^{89}$, G. Qin $^{53}$, Y. Qin ${ }^{84}$, A. Quadt ${ }^{54}$, D. R. Quarrie ${ }^{15}$, W. B. Quayle ${ }^{164 a, 164 b}$, M. Queitsch-Maitland ${ }^{84}$, D. Quilty ${ }^{53}$, S. Raddum ${ }^{119}$, V. Radeka ${ }^{25}$, V. Radescu ${ }^{42}$, S. K. Radhakrishnan ${ }^{148}$, P. Radloff $^{116}$, P. Rados ${ }^{88}$, F. Ragusa ${ }^{91 a}$,91b, G. Rahal ${ }^{178}$, S. Rajagopalan ${ }^{25}$, M. Rammensee ${ }^{30}$, C. Rangel-Smith ${ }^{166}$, F. Rauscher ${ }^{100}$, S. Rave $^{83}$, T. Ravenscroft ${ }^{53}$, M. Raymond ${ }^{30}$, A. L. Read $^{119}$, N. P. Readioff ${ }^{74}$, D. M. Rebuzzi ${ }^{121 a, 121 b}$, A. Redelbach ${ }^{174}$, G. Redlinger $^{25}$, R. Reece ${ }^{137}$, K. Reeves ${ }^{41}$, L. Rehnisch ${ }^{16}$, J. Reichert ${ }^{122}$, H. Reisin ${ }^{27}$, M. Relich ${ }^{163}$, C. Rembser ${ }^{30}$, H. Ren ${ }^{33 a}$, A. Renaud ${ }^{117}$, M. Rescigno ${ }^{132 a}$, S. Resconi ${ }^{91 a}$, O. L. Rezanova ${ }^{109, c}$, P. Reznicek ${ }^{129}$, R. Rezvani ${ }^{95}$, R. Richter ${ }^{101}$ ， S. Richter ${ }^{78}$, E. Richter-Was ${ }^{38 b}$, O. Ricken ${ }^{21}$, M. Ridel ${ }^{80}$ ， P. Rieck ${ }^{16}$ ， C. J. Riegel ${ }^{175}$ ， J. Rieger ${ }^{54}$, O. Rifki113, M. Rijssenbeek ${ }^{148}$, A. Rimoldi ${ }^{121 a, 121 b}$, L. Rinaldi' ${ }^{20 a}$, B. Ristić ${ }^{49}$, E. $\operatorname{Ritsch}^{30}$, I. Riu ${ }^{12}$, F. Rizatdinova ${ }^{114}$, E. Rizvi ${ }^{76}$, S. H. Robertson ${ }^{87, \mathrm{k}}$, A. Robichaud-Veronneau ${ }^{87}, \quad$ D. Robinson ${ }^{28}, \quad$ J. E. M. Robinson ${ }^{42}, \quad$ A. Robson ${ }^{53}$, C. Roda ${ }^{124 a, 124 b}$ ，S. Roe ${ }^{30}$ ，O. Røhne ${ }^{119}$ ，S. Rolli ${ }^{161}$ ，A. Romaniouk ${ }^{98}$ ， M. Romano 20a,20b， S. M. Romano Saez ${ }^{34}$, E. Romero Adam ${ }^{167}$, N. Rompotis ${ }^{138}$, M. Ronzani ${ }^{48}$, L. Roos ${ }^{80}$, E. Ros ${ }^{167}$ ，S. Rosati ${ }^{132 a}$ ，K. Rosbach ${ }^{48}$, P. Rose ${ }^{137}$, P. L. Rosendahl ${ }^{14}$, O. Rosenthal ${ }^{141}$, V. Rossetti ${ }^{146 a, 146 b}$, E. Rossi ${ }^{104 a, 104 b}$, L. P. Rossi ${ }^{50 a}$, J. H. N. Rosten ${ }^{28}$, R. Rosten ${ }^{138}$, M. Rotaru ${ }^{26 a}$ ， I. Roth ${ }^{172}$ ，J. Rothberg ${ }^{138}$ ，D. Rousseau ${ }^{117}$ ，C. R. Royon ${ }^{136}$, A. Rozanov ${ }^{85}$, Y. Rozen ${ }^{152}$, X. Ruan ${ }^{145 c}$, F. Rubbo ${ }^{143}$, I. Rubinskiy ${ }^{42}$, V. I. Rud ${ }^{99}$, C. Rudolph ${ }^{44}$, M. S. Rudolph ${ }^{158}$, F. Rühr ${ }^{48}$, A. Ruiz-Martinez ${ }^{30}$, Z. Rurikova ${ }^{48}$, N. A. Rusakovich ${ }^{65}$, A. Ruschke ${ }^{100}$, H. L. Russell ${ }^{138}$, J. P. Rutherfoord ${ }^{7}$, N. Ruthmannn ${ }^{48}$, Y. F. Ryabov ${ }^{123}$, M. Rybar ${ }^{165}$, G. Rybkin ${ }^{117}$ ， N. C. Ryder ${ }^{120}$ ，A. F. Saavedra ${ }^{150}$, G. Sabato ${ }^{107}$ ，S. Sacerdoti27 ${ }^{27}$ A. Saddique ${ }^{3}$, H. F-W. Sadrozinski ${ }^{137}$, R. Sadykov ${ }^{65}$ ， F. Safai Tehrani ${ }^{132 a}$ ， M. Sahinsoy ${ }^{58 a}$ ， M. Saimpert ${ }^{136}$ ， T. Saito ${ }^{155}$ ， H. Sakamoto ${ }^{155}$ ， Y. Sakurai ${ }^{171}$, G. Salamanna ${ }^{134 a, 134 b}$, A. Salamon ${ }^{133 a}$, J. E. Salazar Loyola ${ }^{32 b}$, M. Saleem ${ }^{113}$ ，D. Salek ${ }^{107}$ ， P. H. Sales De Bruin ${ }^{138}$, D. Salihagic ${ }^{101}$ ， A. Salnikov ${ }^{143}$ ， J. Salt ${ }^{167}$ ， D. Salvatore $37 \mathrm{a}, 37 \mathrm{~b}, \quad$ F. Salvatore ${ }^{149}$, A. Salvucci ${ }^{60 a}$ ， A. Salzburger ${ }^{30}$, D. Sammel ${ }^{48}$, D. Sampsonidis ${ }^{154}$, A. Sanchez ${ }^{104 a, 104 b}, \quad$ J. Sánchez ${ }^{167}, \quad$ V. Sanchez Martinez ${ }^{167}, \quad$ H. Sandaker ${ }^{119}$, R. L. $\operatorname{Sandbach}^{76}$, H. G. $\operatorname{Sander}^{83}$, M. P. Sanders ${ }^{100}$, M. Sandhoff ${ }^{175}$, C. $\operatorname{Sandoval}^{162}$, R. Sandstroem ${ }^{101}$, D. P. C. Sankey ${ }^{131}$, M. Sannino ${ }^{50 a, 50 b}$, A. Sansoni ${ }^{47}$, C. Santoni ${ }^{34}$, R. Santonico ${ }^{133 a, 133 b}$, H. Santos ${ }^{126 a}$, I. Santoyo Castillo ${ }^{149}$, K. Sapp ${ }^{125}$, A. Sapronov ${ }^{65}$ ， J. G. Saraiva ${ }^{126 a, 126 d}$ ， B. Sarrazin ${ }^{21}$, O. Sasaki ${ }^{66}$, Y. Sasaki ${ }^{155}$ ， K. Sato ${ }^{160}$, G. Sauvage, ${ }^{5}$, E. Sauvan ${ }^{5}$, 


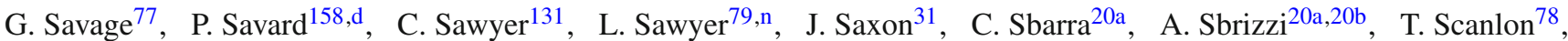
D. A. Scannicchio ${ }^{163}$, M. Scarcella ${ }^{150}$, V. Scarfone ${ }^{37 a, 37 b}$, J. Schaarschmidt ${ }^{172}$, P. Schacht ${ }^{101}$, D. Schaefer ${ }^{30}$, R. Schaefer ${ }^{42}$, J. Schaeffer ${ }^{83}$ ， S. Schaepe ${ }^{21}$ ， S. Schaetzel ${ }^{58 b}$ ， U. Schäfer ${ }^{83}$ ，A. C. Schaffer ${ }^{117}$ ，D. Schaile ${ }^{100}$ ， R. D. Schamberger ${ }^{148}$, V. Scharf ${ }^{58 a}$, V. A. Schegelsky ${ }^{123}$, D. Scheirich ${ }^{129}$, M. Schernau ${ }^{163}$, C. Schiavi ${ }^{50 a, 50 b}$, C. Schillo ${ }^{48}$, M. Schioppa ${ }^{37 a, 37 b}$, S. Schlenker ${ }^{30}$ ， K. Schmieden ${ }^{30}$ ， C. Schmitt ${ }^{83}$ ， S. Schmitt ${ }^{58 b}$ ， S. Schmitt ${ }^{42}$, B. Schneider ${ }^{159 a}$, Y. J. Schnellbach ${ }^{74}$, U. Schnoor ${ }^{44}$, L. Schoeffel ${ }^{136}$, A. Schoening ${ }^{58 b}$, B. D. Schoenrock ${ }^{90}$, E. Schopf ${ }^{21}$, A. L. S. Schorlemmer ${ }^{54}$, M. Schott ${ }^{83}$, D. Schouten ${ }^{159 a}$, J. Schovancova ${ }^{8}$, S. Schramm ${ }^{49}$ ， M. Schreyer ${ }^{174}$ ， C. Schroeder ${ }^{83}$ ， N. Schuh ${ }^{83}$, M. J. Schultens ${ }^{21}$, H.-C. Schultz-Coulon ${ }^{58 a}$, H. Schulz ${ }^{16}$, M. Schumacher ${ }^{48}$, B. A. Schumm ${ }^{137}$, Ph. Schune ${ }^{136}$, C. Schwanenberger ${ }^{84}$, A. Schwartzman ${ }^{143}$, T. A. Schwarz ${ }^{89}$, Ph. Schwegler ${ }^{101}$, H. Schweiger ${ }^{84}$, Ph. Schwemling ${ }^{136}$, R. Schwienhorst ${ }^{90}$, J. Schwindling ${ }^{136}$, T. Schwindt ${ }^{21}$, F. G. Sciacca ${ }^{17}$, E. Scifo ${ }^{117}$ ，G. Sciolla ${ }^{23}$, F. Scuri ${ }^{124 a, 124 b}$, F. Scutti ${ }^{21}$, J. Searcy ${ }^{89}$, G. Sedov ${ }^{42}$, E. Sedykh ${ }^{123}$, P. Seema ${ }^{21}$, S. C. Seidel ${ }^{105}$, A. Seiden ${ }^{137}$, F. Seifert ${ }^{128}$, J. M. Seixas ${ }^{24 a}$, G. Sekhniaidze ${ }^{104 a}$, K. Sekhon ${ }^{89}$, S. J. Sekula ${ }^{40}$, D. M. Seliverstov ${ }^{123, *}$, N. Semprini-Cesari ${ }^{20 a, 20 b}$, C. Serfon ${ }^{30}$, L. Serin ${ }^{117}$, L. Serkin ${ }^{164 a, 164 b}$, T. Serre ${ }^{85}$, M. Sessa ${ }^{134 a, 134 b}$, R. Seuster ${ }^{159 a}$, H. Severini ${ }^{113}$, T. Sfiligoj ${ }^{75}$, F. Sforza ${ }^{30}$, A. Sfyrla ${ }^{30}$, E. Shabalina ${ }^{54}$, M. Shamim ${ }^{116}$, L. Y. Shan ${ }^{33 a}$, R. Shang ${ }^{165}$, J. T. Shank ${ }^{22}$, M. Shapiro ${ }^{15}$, P. B. Shatalov ${ }^{97}$, K. Shaw ${ }^{164 a, 164 b}$, S. M. Shaw ${ }^{84}$, A. Shcherbakova ${ }^{146 a, 146 b}$, C. Y. Shehu ${ }^{149}$, P. Sherwood ${ }^{78}$, L. Shi ${ }^{151, \text { af }, ~ S . ~ S h i m i z u ~}{ }^{67}$, C. O. Shimmin ${ }^{163}$, M. Shimojima ${ }^{102}$, M. Shiyakova ${ }^{65}$, A. Shmeleva ${ }^{96}$, D. Shoaleh Saadi ${ }^{95}$, M. J. Shochet ${ }^{31}$, S. Shojaiii1a,91b, S. Shrestha ${ }^{111}$, E. Shulga ${ }^{98}$, M. A. Shupe ${ }^{7}$, S. Shushkevich ${ }^{42}$, P. Sicho ${ }^{127}$, P. E. Sidebo ${ }^{147}$, O. Sidiropoulou ${ }^{174}$, D. Sidorov ${ }^{114}$, A. Sidoti ${ }^{20 a, 20 b}$, F. Siegert ${ }^{44}$, Dj. Sijacki13 ${ }^{13}$, J. Silva ${ }^{126 a, 126 d}$, Y. Silver ${ }^{153}$, S. B. Silverstein ${ }^{146 a}$, V. Simak ${ }^{128}$, O. Simard ${ }^{5}$, Lj. Simic $^{13}$, S. Simion ${ }^{117}$, E. Simioni ${ }^{83}$, B. Simmons ${ }^{78}$, D. Simon ${ }^{34}$, P. Sinervo ${ }^{158}$, N. B. Sinev ${ }^{116}$, M. Siolin ${ }^{20 a, 20 b}$, G. Siragusa ${ }^{174}$, A. N. Sisakyan ${ }^{65, *}$, S. Yu. Sivoklokov ${ }^{99}$, J. Sjölin ${ }^{146 a, 146 b}, \quad$ T. B. Sjursen ${ }^{14}, \quad$ M. B. Skinner ${ }^{72}, \quad$ H. P. Skottowe ${ }^{57}$, P. Skubic ${ }^{113}$, M. Slater ${ }^{18}$, T. Slavicek ${ }^{128}$, M. Slawinska ${ }^{107}$, K. Sliwa ${ }^{161}$, V. Smakhtin ${ }^{172}$, B. H. Smart ${ }^{46}$, L. Smestad ${ }^{14}$, S. Yu. Smirnov ${ }^{98}$, Y. Smirnov ${ }^{98}$, L. N. Smirnova ${ }^{99, a g}$, O. Smirnova ${ }^{81}$, M. N. K. Smith ${ }^{35}$, R. W. Smith ${ }^{35}$, M. Smizanska ${ }^{72}$,

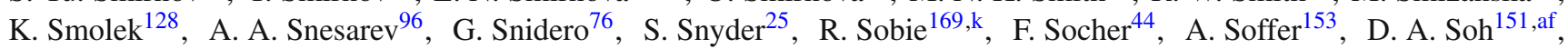
G. Sokhrannyi ${ }^{75}$, C. A. Solans ${ }^{30}$, M. Solar ${ }^{128}$, J. Solc ${ }^{128}$, E. Yu. Soldatov ${ }^{98}$, U. Soldevila ${ }^{167}$, A. A. Solodkov ${ }^{130}$, A. Soloshenko ${ }^{65}$, O. V. Solovyanov ${ }^{130}$, V. Solovyev ${ }^{123}$, P. Sommer ${ }^{48}$, H. Y. Song ${ }^{33 b}$, N. Soni ${ }^{1}$, A. Sood ${ }^{15}$, A. Sopczak ${ }^{128}$, B. Sopko ${ }^{128}$, V. Sopko ${ }^{128}$, V. Sorin ${ }^{12}$, D. Sosa ${ }^{58 b}$, M. Sosebee ${ }^{8}$, C. L. Sotiropoulou ${ }^{124 a, 124 b}$, R. Soualah ${ }^{164 a, 164 c}$, A. M. Soukharev ${ }^{109, c}$, D. South ${ }^{42}$, B. C. Sowden ${ }^{77}$, S. Spagnolo ${ }^{73 a, 73 b}$, M. Spalla ${ }^{124 a, 124 b}$, M. Spangenberg ${ }^{170}$, F. Spanò ${ }^{77}$, W. R. Spearman ${ }^{57}$, D. Sperlich ${ }^{16}$, F. Spettel ${ }^{101}$, R. Spighi ${ }^{20 a}$, G. Spigo ${ }^{30}$, L. A. Spiller ${ }^{88}$, M. Spousta ${ }^{129}$, T. Spreitzer ${ }^{158}$, R. D. St. Denis ${ }^{53, *}$, A. Stabile ${ }^{91 a}$, S. Staerz ${ }^{44}$, J. Stahlman ${ }^{122}$, R. Stamen ${ }^{58 a}$, S. Stamm ${ }^{16}$, E. Stanecka ${ }^{39}$, C. Stanescu ${ }^{134 a}$, M. Stanescu-Bellu ${ }^{42}$, M. M. Stanitzki ${ }^{42}$, S. Stapnes ${ }^{119}$, E. A. Starchenko ${ }^{130}$, J. Stark ${ }^{55}$, P. Staroba ${ }^{127}$, P. Starovoitov ${ }^{58 a}$, R. Staszewski ${ }^{39}$, P. Steinberg ${ }^{25}$, B. Stelzer ${ }^{142}$, H. J. Stelzer ${ }^{30}$, O. Stelzer-Chilton ${ }^{159 a}$, H. Stenzel ${ }^{52}$, G. A. Stewart ${ }^{53}$, J. A. Stillings ${ }^{21}$, M. C. Stockton ${ }^{87}$, M. Stoebe ${ }^{87}$, G. Stoicea ${ }^{26 a}$, P. Stolte ${ }^{54}$, S. Stonjek ${ }^{101}$, A. R. Stradling ${ }^{8}$, A. Straessner ${ }^{44}$, M. E. Stramaglia ${ }^{17}$, J. Strandberg ${ }^{147}$, S. Strandberg ${ }^{146 a, 146 b}$, A. Strandlie ${ }^{119}$, E. Strauss ${ }^{143}$, M. Strauss ${ }^{113}$, P. Strizenec ${ }^{144 b}$, R. Ströhmer ${ }^{174}$, D. M. Strom ${ }^{116}$, R. Stroynowski ${ }^{40}$, A. Strubig ${ }^{106}$, S. A. Stucci ${ }^{17}$, B. Stugu ${ }^{14}$, N. A. Styles ${ }^{42}$, D. Su ${ }^{143}$, J. $\mathrm{Su}^{125}$, R. Subramaniam ${ }^{79}$, A. Succurro ${ }^{12}$, Y. Sugaya ${ }^{118}$, M. Suk ${ }^{128}$, V. V. Sulin ${ }^{96}$, S. Sultansoy ${ }^{4 c}$, T. Sumida ${ }^{68}$, S. Sun ${ }^{57}$, X. Sun ${ }^{33 a}$, J. E. Sundermann ${ }^{48}$, K. Suruliz ${ }^{149}$, G. Susinno ${ }^{37 a, 37 b}$, M. R. Sutton ${ }^{149}$, S. Suzuki ${ }^{66}$, M. Svatos ${ }^{127}$, M. Swiatlowski ${ }^{143}$, I. Sykora ${ }^{144 a}$, T. Sykora ${ }^{129}$, D. Ta ${ }^{48}$, C. Taccini ${ }^{134 a, 134 b}$, K. Tackmann ${ }^{42}$, J. Taenzer ${ }^{158}$, A. Taffard ${ }^{163}$, R. Tafirout ${ }^{159}$, N. Taiblum ${ }^{153}$, H. Takai ${ }^{25}$, R. Takashima ${ }^{69}$, H. Takeda $^{67}$, T. Takeshita ${ }^{140}$, Y. Takubo ${ }^{66}$, M. Talby ${ }^{85}$, A. A. Talyshev ${ }^{109, c}$, J. Y. C. Tam ${ }^{174}$, K. G. Tan ${ }^{88}$, J. Tanaka ${ }^{155}$ ， R. Tanaka ${ }^{117}$ ， S. Tanaka ${ }^{66}$, B. B. Tannenwald ${ }^{111}$, N. Tannoury ${ }^{21}$, S. Tapprogge ${ }^{83}$, S. Tarem ${ }^{152}$, F. Tarrade ${ }^{29}$, G. F. Tartarelli ${ }^{91 a}$, P. Tas ${ }^{129}$, M. Tasevsky ${ }^{127}$, T. Tashiro ${ }^{68}$, E. Tassi ${ }^{37 a, 37 b}$, A. Tavares Delgado ${ }^{126 a, 126 b}$, Y. Tayalati ${ }^{135 d}$, F. E. Taylor ${ }^{94}$, G. N. Taylor ${ }^{88}$, P. T. E. Taylor ${ }^{88}$, W. Taylor ${ }^{159 b}$, F. A. Teischinger ${ }^{30}$, M. Teixeira Dias Castanheira ${ }^{76}$, P. Teixeira-Dias ${ }^{77}$, K. K. Temming ${ }^{48}$, D. Temple ${ }^{142}$, H. Ten Kate ${ }^{30}$, P. K. Teng ${ }^{151}$, J. J. Teoh ${ }^{118}$, F. Tepel ${ }^{175}$, S. Terada ${ }^{66}$, K. Terashi ${ }^{155}$, J. Terron ${ }^{82}$, S. Terzo ${ }^{101}$, M. Testa ${ }^{47}$, R. J. Teuscher ${ }^{158, k}$, T. Theveneaux-Pelzer ${ }^{34}$, J. P. Thomas ${ }^{18}$, J. Thomas-Wilsker ${ }^{77}$, E. N. Thompson ${ }^{35}$, P. D. Thompson ${ }^{18}$, R. J. Thompson ${ }^{84}$, A. S. Thompson ${ }^{53}$, L. A. Thomsen ${ }^{176}$, E. Thomson ${ }^{122}$, M. Thomson ${ }^{28}$, R. P. Thun ${ }^{89,}{ }^{*}$, M. J. Tibbetts ${ }^{15}$, R. E. Ticse Torres ${ }^{85}$, V. O. Tikhomirov ${ }^{96, a h}$, Yu. A. Tikhonov ${ }^{109, c}, \quad$ S. Timoshenko ${ }^{98}, \quad$ E. Tiouchichine ${ }^{85}, \quad$ P. Tipton ${ }^{176}, \quad$ S. Tisserant ${ }^{85}$, K. Todome ${ }^{157}$, T. Todorov ${ }^{5, *}$, S. Todorova-Nova ${ }^{129}$, J. Tojo ${ }^{70}$, S. Tokár ${ }^{144 a}$, K. Tokushuku ${ }^{66}$, K. Tollefson ${ }^{90}$, E. Tolley ${ }^{57}$, L. Tomlinson ${ }^{84}$, M. Tomoto ${ }^{103}$, L. Tompkins ${ }^{143}$,ai, K. Toms ${ }^{105}$, E. Torrence ${ }^{116}$, H. Torres ${ }^{142}$, E. Torró Pastor ${ }^{138}$, J. Toth ${ }^{85}$,aj, F. Touchard ${ }^{85}$, D. R. Tovey ${ }^{139}$, T. Trefzger ${ }^{174}$, L. Tremblet $^{30}$, A. Tricoli ${ }^{30}$, I. M. Trigger ${ }^{159 a}$, S. Trincaz-Duvoid ${ }^{80}$, M. F. Tripiana ${ }^{12}$, W. Trischuk ${ }^{158}$, B. Trocmé ${ }^{55}$, C. Troncon ${ }^{91 a}$, M. Trottier-McDonald ${ }^{15}$, M. Trovatelli ${ }^{169}$, P. True ${ }^{90}$, L. Truong ${ }^{164 a, 164 c}$, M. Trzebinski ${ }^{39}$, A. Trzupek ${ }^{39}$, C. Tsarouchas ${ }^{30}$, J. C-L. Tseng ${ }^{120}$, P. V. Tsiareshka ${ }^{92}$, D. Tsionou ${ }^{154}$, G. Tsipolitis ${ }^{10}$, N. Tsirintanis ${ }^{9}$, S. Tsiskaridze ${ }^{12}$, V. Tsiskaridze ${ }^{48}$, E. G. Tskhadadze ${ }^{51 a}$, I. I. Tsukerman ${ }^{97}$, V. Tsulaia ${ }^{15}$, S. Tsuno ${ }^{66}$, D. Tsybychev ${ }^{148}$, A. Tudorache ${ }^{26 a}$, V. Tudorache ${ }^{26 a}$, A. N. Tuna ${ }^{57}$, S. A. Tupputi ${ }^{20 a, 20 b}$, S. Turchikhin ${ }^{99, a g}$, 
D. Turecek ${ }^{128}$ ， R. Turra ${ }^{91 a, 91 b}, \quad$ A. J. Turvey ${ }^{40}, \quad$ P. M. Tuts ${ }^{35}, \quad$ A. Tykhonov ${ }^{49}, \quad$ M. Tylmad ${ }^{146 a, 146 b}, \quad$ M. Tyndel ${ }^{131}$, I. Ueda ${ }^{155}$ ， R. Ueno ${ }^{29}$ ， M. Ughetto ${ }^{146 a, 146 b}$ ， M. Ugland ${ }^{14}$ ，F. Ukegawa ${ }^{160}$ ，G. Unal ${ }^{30}$ ，A. Undrus ${ }^{25}$ ， G. Unel ${ }^{163}$, F. C. Ungaro ${ }^{48}$, Y. Unno ${ }^{66}$, C. Unverdorben ${ }^{100}$, J. Urban ${ }^{144 b}$, P. Urquijo ${ }^{88}$, P. Urrejola ${ }^{83}$, G. Usai ${ }^{8}$, A. Usanova ${ }^{62}$,

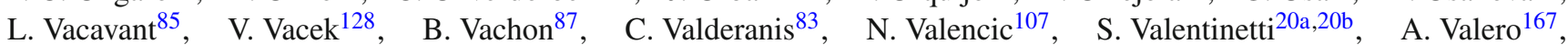
L. Valery ${ }^{12}$, S. Valkar ${ }^{129}$, E. Valladolid Gallego ${ }^{167}$, S. Vallecorsa ${ }^{49}$, J. A. Valls Ferrer ${ }^{167}$, W. Van Den Wollenberg ${ }^{107}$, P. C. Van Der Deij1 ${ }^{107}$, R. van der Geer ${ }^{107}$, H. van der Graaf ${ }^{107}$, N. van Eldik ${ }^{152}$, P. van Gemmeren ${ }^{6}$, J. Van Nieuwkoop ${ }^{142}$, I. van Vulpen ${ }^{107}$, M. C. van Woerden ${ }^{30}$, M. Vanadia ${ }^{132 a, 132 b}$, W. Vandelli ${ }^{30}$, R. Vanguri ${ }^{122}$, A. Vaniachine ${ }^{6}$, F. Vannucci ${ }^{80}$, G. Vardanyan ${ }^{177}$, R. Vari ${ }^{132 a}$, E. W. Varnes ${ }^{7}$, T. Varol $^{40}$, D. Varouchas ${ }^{80}$, A. Vartapetian ${ }^{8}$, K. E. Varvell ${ }^{150}$, F. Vazeille ${ }^{34}$, T. Vazquez Schroeder ${ }^{87}$, J. Veatch ${ }^{7}$, L. M. Veloce ${ }^{158}$, F. Veloso ${ }^{126 a, 126 c}$, T. Velz ${ }^{21}$, S. Veneziano ${ }^{132 a}$, A. Ventura ${ }^{73 a, 73 b}$, D. Ventura ${ }^{86}$, M. Venturi ${ }^{169}$, N. Venturi ${ }^{158}$, A. Venturini ${ }^{23}$, V. Vercesi ${ }^{121 a}$, M. Verducci ${ }^{132 a, 132 b}$, W. Verkerke ${ }^{107}$, J. C. Vermeulen ${ }^{107}$, A. Vest ${ }^{44}$, M. C. Vetterli ${ }^{142, d}$, O. Viazlo ${ }^{81}$, I. Vichou ${ }^{165}$, T. Vickey ${ }^{139}$, O. E. Vickey Boeriu ${ }^{139}$, G. H. A. Viehhauser ${ }^{120}$, S. Viel ${ }^{15}$, R. Vigne ${ }^{62}$, M. Villa ${ }^{20 a, 20 b}$, M. Villaplana Perez ${ }^{91 a, 91 b}$, E. Vilucchi ${ }^{47}$, M. G. Vincter ${ }^{29}$, V. B. Vinogradov ${ }^{65}$ ， I. Vivarelli ${ }^{149}$ ， F. Vives Vaque ${ }^{3} ， \quad$ S. Vlachos ${ }^{10}, \quad$ D. Vladoiu ${ }^{100}, \quad$ M. Vlasak ${ }^{128}, \quad$ M. Vogel $^{32 a}$, P. Vokac ${ }^{128}$, G. Volpi ${ }^{124 a, 124 b}$, M. Volpi ${ }^{88}$, H. von der Schmitt ${ }^{101}$, H. von Radziewski ${ }^{48}$, E. von Toerne ${ }^{21}$, V. Vorobel ${ }^{129}$, K. Vorobev ${ }^{98}, \quad$ M. $\operatorname{Vos}^{167}, \quad$ R. Voss ${ }^{30}, \quad$ J. H. Vossebeld ${ }^{74}, \quad$ N. Vranjes ${ }^{13}, \quad$ M. Vranjes Milosavljevic ${ }^{13}, \quad$ V. Vrba ${ }^{127}$, M. Vreeswijk ${ }^{107}$, R. Vuillermet ${ }^{30}$, I. Vukotic ${ }^{31}$, Z. Vykydal ${ }^{128}$, P. Wagner ${ }^{21}$, W. Wagner ${ }^{175}$, H. Wahlberg ${ }^{71}$, S. Wahrmund ${ }^{44}$, J. Wakabayashi ${ }^{103}$, J. Walder ${ }^{72}$, R. Walker ${ }^{100}$, W. Walkowiak ${ }^{141}$, C. Wang ${ }^{151}$, F. Wang ${ }^{173}$, H. Wang ${ }^{15}$, H. Wang $^{40}$ J. Wang $^{42}$,

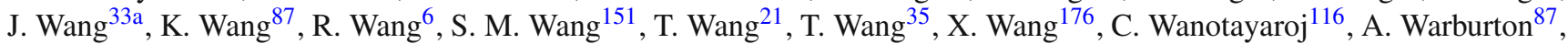
C. P. Ward ${ }^{28}$, D. R. Wardrope ${ }^{78}$, A. Washbrook ${ }^{46}$, C. Wasicki ${ }^{42}$, P. M. Watkins ${ }^{18}$, A. T. Watson ${ }^{18}$, I. J. Watson ${ }^{150}$, M. F. Watson ${ }^{18}$, G. Watts ${ }^{138}$, S. Watts ${ }^{84}$, B. M. Waugh ${ }^{78}$, S. Webb ${ }^{84}$, M. S. Weber ${ }^{17}$, S. W. Weber ${ }^{174}$, J. S. Webster ${ }^{31}$, A. R. Weidberg ${ }^{120}$, B. Weinert ${ }^{61}$, J. Weingarten ${ }^{54}$, C. Weiser ${ }^{48}$, H. Weits ${ }^{107}$, P. S. Wells ${ }^{30}$, T. Wenaus ${ }^{25}$, T. Wengler ${ }^{30}$, S. Wenig ${ }^{30}$, N. Wermes ${ }^{21}$, M. Werner ${ }^{48}$, P. Werner ${ }^{30}$, M. Wessels ${ }^{58 a}$, J. Wetter ${ }^{161}$, K. Whalen ${ }^{116}$, A. M. Wharton ${ }^{72}$, A. White ${ }^{8}$, M. J. White ${ }^{1}$, R. White ${ }^{32 b}$, S. White ${ }^{124 a, 124 b}$, D. Whiteson ${ }^{163}$, F. J. Wickens ${ }^{131}$, W. Wiedenmann ${ }^{173}$,

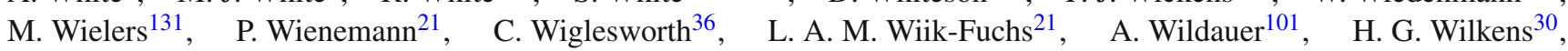
H. H. Williams ${ }^{122}$, S. Williams ${ }^{107}$, C. Willis ${ }^{90}, \quad$ S. Willocq ${ }^{86}, \quad$ A. Wilson ${ }^{89}$, J. A. Wilson ${ }^{18}$, I. Wingerter-Seez ${ }^{5}$, F. Winklmeier ${ }^{116}$, B. T. Winter ${ }^{21}$, M. Wittgen ${ }^{143}$, J. Wittkowski ${ }^{100}$, S. J. Wollstadt ${ }^{83}$, M. W. Wolter ${ }^{39}$, H. Wolters ${ }^{126 a, 126 c}$, B. K. Wosiek ${ }^{39}$, J. Wotschack ${ }^{30}$, M. J. Woudstra ${ }^{84}$, K. W. Wozniak ${ }^{39}$, M. Wu ${ }^{55}$, M. Wu ${ }^{31}$, S. L. Wu ${ }^{173}, \mathrm{X} \mathrm{Wu}^{49}, \mathrm{Y} \mathrm{Wu}^{89}$, T. R. Wyatt ${ }^{84}$, B. M. Wynne ${ }^{46}$, S. Xella ${ }^{36}$, D. Xu ${ }^{33 a}$, L. Xu ${ }^{25}$, B. Yabsley ${ }^{150}$, S. Yacoob ${ }^{145 a}$, R. Yakabe ${ }^{67}$, M. Yamada ${ }^{66}$, D. Yamaguchi ${ }^{157}$, Y. Yamaguchi ${ }^{118}$, A. Yamamoto ${ }^{66}$, S. Yamamoto ${ }^{155}$, T. Yamanaka ${ }^{155}$, K. Yamauchi ${ }^{103}$, Y. Yamazaki ${ }^{67}$, Z. Yan ${ }^{22}$, H. Yang ${ }^{33 e}$, H. Yang ${ }^{173}$, Y. Yang ${ }^{151}$, W-M. Yao ${ }^{15}$, Y. Yasu ${ }^{66}$, E. Yatsenko ${ }^{5}$, K. H. Yau Wong ${ }^{21}$, J. Ye $^{40}$, S. Ye $^{25}$, I. Yeletskikh ${ }^{65}$, A. L. Yen ${ }^{57}$, E. Yildirim ${ }^{42}$, K. Yorita ${ }^{171}$, R. Yoshida ${ }^{6}$, K. Yoshihara ${ }^{122}$, C. Young ${ }^{143}$, C. J. S. Young ${ }^{30}$, S. Youssef ${ }^{22}$, D. R. Yu ${ }^{15}$, J. Yu ${ }^{8}$, J. M. Yu ${ }^{89}$, J. Yu ${ }^{114}$, L. Yuan ${ }^{67}$, S. P. Y. Yuen ${ }^{21}$, A. Yurkewicz ${ }^{108}$, I. Yusuff ${ }^{28, a k}$, B. Zabinski ${ }^{39}$, R. Zaidan ${ }^{63}$, A. M. Zaitsev ${ }^{130, a b}$, J. Zalieckas ${ }^{14}$, A. Zaman ${ }^{148}$, S. Zambito $^{57}$, L. Zanello ${ }^{132 a, 132 b}$, D. Zanzi ${ }^{88}$, C. Zeitnitz ${ }^{175}$, M. Zeman ${ }^{128}$, A. Zemla ${ }^{38 a}$, Q. Zeng ${ }^{143}$, K. Zengel $^{23}$, O. Zenin ${ }^{130}$, T. Ženišs ${ }^{144 a}$, D. Zerwas ${ }^{117}$, D. Zhang ${ }^{89}$, F. Zhang ${ }^{173}$, H. Zhang ${ }^{33 c}$, J. Zhang ${ }^{6}$, L. Zhang ${ }^{48}$, R. Zhang ${ }^{33 b, i}$, X. Zhang ${ }^{33 d}$, Z. Zhang ${ }^{117}$, X. Zhao ${ }^{40}$, Y. Zhao ${ }^{33 d, 117}$, Z. Zhao ${ }^{33 b}$, A. Zhemchugov ${ }^{65}$, J. Zhong ${ }^{120}$, B. Zhou ${ }^{89}$, C. Zhou ${ }^{45}$, L. Zhou ${ }^{35}$, L. Zhou ${ }^{40}$, M. Zhou ${ }^{148}$, N. Zhou ${ }^{33 f}$, C. G. Zhu ${ }^{33 d}$, H. Zhu ${ }^{33 a}$, J. Zhu ${ }^{89}$, Y. Zhu ${ }^{33 b}$, X. Zhuang ${ }^{33 a}$, K. Zhukov ${ }^{96}$, A. Zibell ${ }^{174}$, D. Zieminska ${ }^{61}$, N. I. Zimine ${ }^{65}$, C. Zimmermann ${ }^{83}$, S. Zimmermann ${ }^{48}$, Z. Zinonos ${ }^{54}$, M. Zinser ${ }^{83}$, M. Ziolkowski ${ }^{141}$, L. Živković ${ }^{13}$, G. Zobernig ${ }^{173}$, A. Zoccoli ${ }^{20 a, 20 b}$, M. zur Nedden ${ }^{16}$, G. Zurzolo ${ }^{104 a, 104 b}$, L. Zwalinski $^{30}$

${ }^{1}$ Department of Physics, University of Adelaide, Adelaide, Australia

${ }^{2}$ Physics Department, SUNY Albany, Albany, NY, USA

${ }^{3}$ Department of Physics, University of Alberta, Edmonton, AB, Canada

4 (a) Department of Physics, Ankara University, Ankara, Turkey; ${ }^{(b)}$ Istanbul Aydin University, Istanbul, Turkey; ${ }^{(c)}$ Division of Physics, TOBB University of Economics and Technology, Ankara, Turkey

${ }^{5}$ LAPP, CNRS/IN2P3 and Université Savoie Mont Blanc, Annecy-le-Vieux, France

${ }^{6}$ High Energy Physics Division, Argonne National Laboratory, Argonne, IL, USA

${ }^{7}$ Department of Physics, University of Arizona, Tucson, AZ, USA

${ }^{8}$ Department of Physics, The University of Texas at Arlington, Arlington, TX, USA

${ }^{9}$ Physics Department, University of Athens, Athens, Greece

${ }^{10}$ Physics Department, National Technical University of Athens, Zografou, Greece

${ }^{11}$ Institute of Physics, Azerbaijan Academy of Sciences, Baku, Azerbaijan

${ }^{12}$ Institut de Física d'Altes Energies and Departament de Física de la Universitat Autònoma de Barcelona, Barcelona, Spain 
${ }^{13}$ Institute of Physics, University of Belgrade, Belgrade, Serbia

${ }^{14}$ Department for Physics and Technology, University of Bergen, Bergen, Norway

${ }^{15}$ Physics Division, Lawrence Berkeley National Laboratory and University of California, Berkeley, CA, USA

${ }^{16}$ Department of Physics, Humboldt University, Berlin, Germany

${ }^{17}$ Albert Einstein Center for Fundamental Physics and Laboratory for High Energy Physics, University of Bern, Bern, Switzerland

${ }^{18}$ School of Physics and Astronomy, University of Birmingham, Birmingham, UK

19 (a) Department of Physics, Bogazici University, Istanbul, Turkey; ${ }^{(b)}$ Department of Physics Engineering, Gaziantep

University, Gaziantep, Turkey; ${ }^{\left({ }^{c}\right)}$ Department of Physics, Dogus University, Istanbul, Turkey

20 (a) INFN Sezione di Bologna, Bologna, Italy; ${ }^{(b)}$ Dipartimento di Fisica e Astronomia, Università di Bologna, Bologna, Italy

${ }^{21}$ Physikalisches Institut, University of Bonn, Bonn, Germany

22 Department of Physics, Boston University, Boston, MA, USA

${ }^{23}$ Department of Physics, Brandeis University, Waltham, MA, USA

24 (a) Universidade Federal do Rio De Janeiro COPPE/EE/IF, Rio de Janeiro, Brazil; ${ }^{(b)}$ Electrical Circuits Department, Federal University of Juiz de Fora (UFJF), Juiz de Fora, Brazil; ${ }^{\left({ }^{c}\right)}$ Federal University of Sao Joao del Rei (UFSJ), Sao

Joao del Rei, Brazil; ${ }^{(d)}$ Instituto de Fisica, Universidade de Sao Paulo, São Paulo, Brazil

${ }^{25}$ Physics Department, Brookhaven National Laboratory, Upton, NY, USA

26 (a) National Institute of Physics and Nuclear Engineering, Bucharest, Romania; ${ }^{(b)}$ Physics Department, National Institute for Research and Development of Isotopic and Molecular Technologies, Cluj Napoca, Romania; ${ }^{\left({ }^{c}\right)}$ University Politehnica Bucharest, Bucharest, Romania; ${ }^{(d)}$ West University in Timisoara, Timisoara, Romania

${ }^{27}$ Departamento de Física, Universidad de Buenos Aires, Buenos Aires, Argentina

${ }^{28}$ Cavendish Laboratory, University of Cambridge, Cambridge, UK

${ }^{29}$ Department of Physics, Carleton University, Ottawa, ON, Canada

${ }^{30}$ CERN, Geneva, Switzerland

${ }^{31}$ Enrico Fermi Institute, University of Chicago, Chicago, IL, USA

32 (a) Departamento de Física, Pontificia Universidad Católica de Chile, Santiago, Chile; (b) Departamento de Física, Universidad Técnica Federico Santa María, Valparaiso, Chile

33 (a) Institute of High Energy Physics, Chinese Academy of Sciences, Beijing, China; ${ }^{(b)}$ Department of Modern Physics, University of Science and Technology of China, Hefei, Anhui, China; ${ }^{(c)}$ Department of Physics, Nanjing University, Nanjing, Jiangsu, China; ${ }^{(d)}$ School of Physics, Shandong University, Jinan, Shandong, China; ${ }^{(e)}$ Shanghai Key Laboratory for Particle Physics and Cosmology, Department of Physics and Astronomy, Shanghai Jiao Tong University, Shanghai, China; ${ }^{(\mathrm{f})}$ Physics Department, Tsinghua University, Beijing 100084, China

${ }^{34}$ Laboratoire de Physique Corpusculaire, Clermont Université and Université Blaise Pascal and CNRS/IN2P3, Clermont-Ferrand, France

${ }^{35}$ Nevis Laboratory, Columbia University, Irvington, NY, USA

${ }^{36}$ Niels Bohr Institute, University of Copenhagen, Copenhagen, Denmark

37 (a) INFN Gruppo Collegato di Cosenza, Laboratori Nazionali di Frascati, Frascati, Italy; ${ }^{(b)}$ Dipartimento di Fisica, Università della Calabria, Rende, Italy

38 (a) Faculty of Physics and Applied Computer Science, AGH University of Science and Technology, Kraków, Poland; (b) Marian Smoluchowski Institute of Physics, Jagiellonian University, Kraków, Poland

${ }^{39}$ Institute of Nuclear Physics, Polish Academy of Sciences, Kraków, Poland

${ }^{40}$ Physics Department, Southern Methodist University, Dallas, TX, USA

${ }^{41}$ Physics Department, University of Texas at Dallas, Richardson, TX, USA

${ }^{42}$ DESY, Hamburg and Zeuthen, Germany

${ }^{43}$ Institut für Experimentelle Physik IV, Technische Universität Dortmund, Dortmund, Germany

${ }^{44}$ Institut für Kern- und Teilchenphysik, Technische Universität Dresden, Dresden, Germany

${ }^{45}$ Department of Physics, Duke University, Durham, NC, USA

46 SUPA-School of Physics and Astronomy, University of Edinburgh, Edinburgh, UK

${ }^{47}$ INFN Laboratori Nazionali di Frascati, Frascati, Italy

${ }^{48}$ Fakultät für Mathematik und Physik, Albert-Ludwigs-Universität, Freiburg, Germany

${ }^{49}$ Section de Physique, Université de Genève, Geneva, Switzerland

50 (a) INFN Sezione di Genova, Genoa, Italy; ${ }^{(b)}$ Dipartimento di Fisica, Università di Genova, Genoa, Italy 
51 (a) E. Andronikashvili Institute of Physics, Iv. Javakhishvili Tbilisi State University, Tbilisi, Georgia; ${ }^{(b)}$ High Energy Physics Institute, Tbilisi State University, Tbilisi, Georgia

52 II Physikalisches Institut, Justus-Liebig-Universität Giessen, Giessen, Germany

${ }^{53}$ SUPA-School of Physics and Astronomy, University of Glasgow, Glasgow, UK

54 II Physikalisches Institut, Georg-August-Universität, Göttingen, Germany

${ }^{55}$ Laboratoire de Physique Subatomique et de Cosmologie, Université Grenoble-Alpes, CNRS/IN2P3, Grenoble, France

${ }^{56}$ Department of Physics, Hampton University, Hampton, VA, USA

${ }^{57}$ Laboratory for Particle Physics and Cosmology, Harvard University, Cambridge, MA, USA

58 (a) Kirchhoff-Institut für Physik, Ruprecht-Karls-Universität Heidelberg, Heidelberg, Germany; ${ }^{(b)}$ Physikalisches Institut, Ruprecht-Karls-Universität Heidelberg, Heidelberg, Germany; ${ }^{(c)}$ ZITI Institut für technische Informatik,

Ruprecht-Karls-Universität Heidelberg, Mannheim, Germany

${ }^{59}$ Faculty of Applied Information Science, Hiroshima Institute of Technology, Hiroshima, Japan

60 (a) Department of Physics, The Chinese University of Hong Kong, Shatin, NT, Hong Kong; (b) Department of Physics,

The University of Hong Kong, Pokfulam, Hong Kong; ${ }^{\left({ }^{c}\right)}$ Department of Physics, The Hong Kong University of Science and Technology, Clear Water Bay, Kowloon, Hong Kong, China

${ }^{61}$ Department of Physics, Indiana University, Bloomington, IN, USA

${ }^{62}$ Institut für Astro- und Teilchenphysik, Leopold-Franzens-Universität, Innsbruck, Austria

${ }^{63}$ University of Iowa, Iowa City, IA, USA

${ }^{64}$ Department of Physics and Astronomy, Iowa State University, Ames, IA, USA

65 Joint Institute for Nuclear Research, JINR Dubna, Dubna, Russia

${ }^{66}$ KEK, High Energy Accelerator Research Organization, Tsukuba, Japan

${ }^{67}$ Graduate School of Science, Kobe University, Kobe, Japan

${ }^{68}$ Faculty of Science, Kyoto University, Kyoto, Japan

${ }^{69}$ Kyoto University of Education, Kyoto, Japan

${ }^{70}$ Department of Physics, Kyushu University, Fukuoka, Japan

${ }^{71}$ Instituto de Física La Plata, Universidad Nacional de La Plata and CONICET, La Plata, Argentina

72 Physics Department, Lancaster University, Lancaster, UK

73 (a) INFN Sezione di Lecce, Lecce, Italy; ${ }^{\left({ }^{b}\right)}$ Dipartimento di Matematica e Fisica, Università del Salento, Lecce, Italy

74 Oliver Lodge Laboratory, University of Liverpool, Liverpool, UK

${ }^{75}$ Department of Physics, Jožef Stefan Institute and University of Ljubljana, Ljubljana, Slovenia

${ }^{76}$ School of Physics and Astronomy, Queen Mary University of London, London, UK

77 Department of Physics, Royal Holloway University of London, Surrey, UK

78 Department of Physics and Astronomy, University College London, London, UK

${ }^{79}$ Louisiana Tech University, Ruston, LA, USA

${ }^{80}$ Laboratoire de Physique Nucléaire et de Hautes Energies, UPMC and Université Paris-Diderot and CNRS/IN2P3, Paris, France

${ }^{81}$ Fysiska institutionen, Lunds universitet, Lund, Sweden

82 Departamento de Fisica Teorica C-15, Universidad Autonoma de Madrid, Madrid, Spain

${ }^{83}$ Institut für Physik, Universität Mainz, Mainz, Germany

${ }^{84}$ School of Physics and Astronomy, University of Manchester, Manchester, UK

${ }^{85}$ CPPM, Aix-Marseille Université and CNRS/IN2P3, Marseille, France

86 Department of Physics, University of Massachusetts, Amherst, MA, USA

${ }^{87}$ Department of Physics, McGill University, Montreal, QC, Canada

${ }^{88}$ School of Physics, University of Melbourne, Victoria, Australia

${ }^{89}$ Department of Physics, The University of Michigan, Ann Arbor, MI, USA

${ }^{90}$ Department of Physics and Astronomy, Michigan State University, East Lansing, MI, USA

91 (a) INFN Sezione di Milano, Milan, Italy; ${ }^{(b)}$ Dipartimento di Fisica, Università di Milano, Milan, Italy

92 B.I. Stepanov Institute of Physics, National Academy of Sciences of Belarus, Minsk, Republic of Belarus

${ }^{93}$ National Scientific and Educational Centre for Particle and High Energy Physics, Minsk, Republic of Belarus

${ }^{94}$ Department of Physics, Massachusetts Institute of Technology, Cambridge, MA, USA

${ }^{95}$ Group of Particle Physics, University of Montreal, Montreal, QC, Canada

96 P.N. Lebedev Institute of Physics, Academy of Sciences, Moscow, Russia

${ }^{97}$ Institute for Theoretical and Experimental Physics (ITEP), Moscow, Russia 
${ }^{98}$ National Research Nuclear University MEPhI, Moscow, Russia

${ }^{99}$ D.V. Skobeltsyn Institute of Nuclear Physics, M.V. Lomonosov Moscow State University, Moscow, Russia

${ }^{100}$ Fakultät für Physik, Ludwig-Maximilians-Universität München, Munich, Germany

${ }^{101}$ Max-Planck-Institut für Physik (Werner-Heisenberg-Institut), Munich, Germany

${ }^{102}$ Nagasaki Institute of Applied Science, Nagasaki, Japan

${ }^{103}$ Graduate School of Science and Kobayashi-Maskawa Institute, Nagoya University, Nagoya, Japan

104 (a) INFN Sezione di Napoli, Naples, Italy; ${ }^{(b)}$ Dipartimento di Fisica, Università di Napoli, Naples, Italy

105 Department of Physics and Astronomy, University of New Mexico, Albuquerque, NM, USA

${ }^{106}$ Institute for Mathematics, Astrophysics and Particle Physics, Radboud University Nijmegen/Nikhef, Nijmegen, The

Netherlands

${ }^{107}$ Nikhef National Institute for Subatomic Physics and University of Amsterdam, Amsterdam, The Netherlands

108 Department of Physics, Northern Illinois University, De Kalb, IL, USA

${ }^{109}$ Budker Institute of Nuclear Physics, SB RAS, Novosibirsk, Russia

${ }^{110}$ Department of Physics, New York University, New York, NY, USA

111 Ohio State University, Columbus, OH, USA

112 Faculty of Science, Okayama University, Okayama, Japan

${ }^{113}$ Homer L. Dodge Department of Physics and Astronomy, University of Oklahoma, Norman, OK, USA

${ }^{114}$ Department of Physics, Oklahoma State University, Stillwater, OK, USA

115 Palacký University, RCPTM, Olomouc, Czech Republic

116 Center for High Energy Physics, University of Oregon, Eugene, OR, USA

${ }^{117}$ LAL, Université Paris-Sud and CNRS/IN2P3, Orsay, France

118 Graduate School of Science, Osaka University, Osaka, Japan

${ }^{119}$ Department of Physics, University of Oslo, Oslo, Norway

${ }^{120}$ Department of Physics, Oxford University, Oxford, UK

121 (a) INFN Sezione di Pavia, Pavia, Italy; ${ }^{(b)}$ Dipartimento di Fisica, Università di Pavia, Pavia, Italy

122 Department of Physics, University of Pennsylvania, Philadelphia, PA, USA

${ }^{123}$ National Research Centre "Kurchatov Institute" B.P.Konstantinov Petersburg Nuclear Physics Institute, St. Petersburg, Russia

124 (a) INFN Sezione di Pisa, Pisa, Italy; ${ }^{(b)}$ Dipartimento di Fisica E. Fermi, Università di Pisa, Pisa, Italy

125 Department of Physics and Astronomy, University of Pittsburgh, Pittsburgh, PA, USA

126 (a) Laboratório de Instrumentação e Física Experimental de Partículas-LIP, Lisbon, Portugal; ${ }^{(b)}$ Faculdade de Ciências, Universidade de Lisboa, Lisbon, Portugal; ${ }^{(c)}$ Department of Physics, University of Coimbra, Coimbra, Portugal; ${ }^{\text {(d) }}$ Centro de Física Nuclear da Universidade de Lisboa, Lisbon, Portugal; ${ }^{(e)}$ Departamento de Fisica, Universidade do Minho, Braga, Portugal; ${ }^{(f)}$ Departamento de Fisica Teorica y del Cosmos and CAFPE, Universidad de Granada, Granada, Spain; ${ }^{(g)}$ Dep Fisica and CEFITEC of Faculdade de Ciencias e Tecnologia, Universidade Nova de Lisboa, Caparica, Portugal

${ }^{127}$ Institute of Physics, Academy of Sciences of the Czech Republic, Prague, Czech Republic

${ }^{128}$ Czech Technical University in Prague, Prague, Czech Republic

${ }^{129}$ Faculty of Mathematics and Physics, Charles University in Prague, Prague, Czech Republic

${ }^{130}$ State Research Center Institute for High Energy Physics, Protvino, Russia

131 Particle Physics Department, Rutherford Appleton Laboratory, Didcot, UK

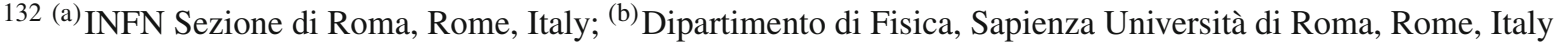

133 (a) INFN Sezione di Roma Tor Vergata, Rome, Italy; ${ }^{(b)}$ Dipartimento di Fisica, Università di Roma Tor Vergata, Rome, Italy

134 (a) INFN Sezione di Roma Tre, Rome, Italy; ${ }^{(b)}$ Dipartimento di Matematica e Fisica, Università Roma Tre, Rome, Italy

135 (a) Faculté des Sciences Ain Chock, Réseau Universitaire de Physique des Hautes Energies-Université Hassan II, Casablanca, Morocco; ${ }^{\left({ }^{b}\right)}$ Centre National de l'Energie des Sciences Techniques Nucleaires, Rabat, Morocco; ${ }^{\left({ }^{(c)}\right.}$ Faculté des Sciences Semlalia, Université Cadi Ayyad, LPHEA-Marrakech, Marrakech, Morocco; ${ }^{(d)}$ Faculté des Sciences, Université Mohamed Premier and LPTPM, Oujda, Morocco; ${ }^{(e)}$ Faculté des Sciences, Université Mohammed V, Rabat, Morocco

${ }^{136}$ DSM/IRFU (Institut de Recherches sur les Lois Fondamentales de l'Univers), CEA Saclay (Commissariat à l'Energie Atomique et aux Energies Alternatives), Gif-sur-Yvette, France

${ }^{137}$ Santa Cruz Institute for Particle Physics, University of California Santa Cruz, Santa Cruz, CA, USA 
${ }^{138}$ Department of Physics, University of Washington, Seattle, WA, USA

${ }^{139}$ Department of Physics and Astronomy, University of Sheffield, Sheffield, UK

${ }^{140}$ Department of Physics, Shinshu University, Nagano, Japan

${ }^{141}$ Fachbereich Physik, Universität Siegen, Siegen, Germany

142 Department of Physics, Simon Fraser University, Burnaby, BC, Canada

${ }^{143}$ SLAC National Accelerator Laboratory, Stanford, CA, USA

144 (a) Faculty of Mathematics, Physics and Informatics, Comenius University, Bratislava, Slovak Republic; ${ }^{(b)}$ Department of Subnuclear Physics, Institute of Experimental Physics of the Slovak Academy of Sciences, Kosice, Slovak Republic

145 (a) Department of Physics, University of Cape Town, Cape Town, South Africa; ${ }^{(b)}$ Department of Physics, University of Johannesburg, Johannesburg, South Africa; ${ }^{(\mathrm{c})}$ School of Physics, University of the Witwatersrand, Johannesburg, South Africa

146 (a) Department of Physics, Stockholm University, Stockholm, Sweden; ${ }^{(b)}$ The Oskar Klein Centre, Stockholm, Sweden

147 Physics Department, Royal Institute of Technology, Stockholm, Sweden

148 Departments of Physics and Astronomy and Chemistry, Stony Brook University, Stony Brook, NY, USA

${ }^{149}$ Department of Physics and Astronomy, University of Sussex, Brighton, UK

${ }^{150}$ School of Physics, University of Sydney, Sydney, Australia

${ }^{151}$ Institute of Physics, Academia Sinica, Taipei, Taiwan

152 Department of Physics, Technion: Israel Institute of Technology, Haifa, Israel

${ }^{153}$ Raymond and Beverly Sackler School of Physics and Astronomy, Tel Aviv University, Tel Aviv, Israel

154 Department of Physics, Aristotle University of Thessaloniki, Thessaloníki, Greece

155 International Center for Elementary Particle Physics and Department of Physics, The University of Tokyo, Tokyo, Japan

${ }^{156}$ Graduate School of Science and Technology, Tokyo Metropolitan University, Tokyo, Japan

${ }^{157}$ Department of Physics, Tokyo Institute of Technology, Tokyo, Japan

${ }^{158}$ Department of Physics, University of Toronto, Toronto, ON, Canada

159 (a) TRIUMF, Vancouver, BC, Canada; ${ }^{(b)}$ Department of Physics and Astronomy, York University, Toronto, ON, Canada

${ }^{160}$ Faculty of Pure and Applied Sciences, University of Tsukuba, Tsukuba, Japan

${ }^{161}$ Department of Physics and Astronomy, Tufts University, Medford, MA, USA

${ }^{162}$ Centro de Investigaciones, Universidad Antonio Narino, Bogotá, Colombia

163 Department of Physics and Astronomy, University of California Irvine, Irvine, CA, USA

164 (a) INFN Gruppo Collegato di Udine, Sezione di Trieste, Udine, Italy; ${ }^{\left({ }^{b}\right)}$ ICTP, Trieste, Italy; ${ }^{(c)}$ Dipartimento di Chimica Fisica e Ambiente, Università di Udine, Udine, Italy

${ }^{165}$ Department of Physics, University of Illinois, Urbana, IL, USA

${ }^{166}$ Department of Physics and Astronomy, University of Uppsala, Uppsala, Sweden

${ }^{167}$ Instituto de Física Corpuscular (IFIC) and Departamento de Física Atómica, Molecular y Nuclear and Departamento de Ingeniería Electrónica and Instituto de Microelectrónica de Barcelona (IMB-CNM), University of Valencia and CSIC, Valencia, Spain

${ }^{168}$ Department of Physics, University of British Columbia, Vancouver, BC, Canada

${ }^{169}$ Department of Physics and Astronomy, University of Victoria, Victoria, BC, Canada

${ }^{170}$ Department of Physics, University of Warwick, Coventry, UK

${ }^{171}$ Waseda University, Tokyo, Japan

172 Department of Particle Physics, The Weizmann Institute of Science, Rehovot, Israel

173 Department of Physics, University of Wisconsin, Madison, WI, USA

${ }^{174}$ Fakultät für Physik und Astronomie, Julius-Maximilians-Universität, Würzburg, Germany

${ }^{175}$ Fachbereich C Physik, Bergische Universität Wuppertal, Wuppertal, Germany

${ }^{176}$ Department of Physics, Yale University, New Haven, CT, USA

177 Yerevan Physics Institute, Yerevan, Armenia

${ }^{178}$ Centre de Calcul de l'Institut National de Physique Nucléaire et de Physique des Particules (IN2P3), Villeurbanne, France

a Also at Department of Physics, King's College London, London, UK

${ }^{b}$ Also at Institute of Physics, Azerbaijan Academy of Sciences, Baku, Azerbaijan

${ }^{\mathrm{c}}$ Also at Novosibirsk State University, Novosibirsk, Russia

d Also at TRIUMF, Vancouver, BC, Canada

e Also at Department of Physics, California State University, Fresno, CA, USA 
${ }^{f}$ Also at Department of Physics, University of Fribourg, Fribourg, Switzerland

g Also at Departamento de Fisica e Astronomia, Faculdade de Ciencias, Universidade do Porto, Porto, Portugal

h Also at Tomsk State University, Tomsk, Russia

${ }^{i}$ Also at CPPM, Aix-Marseille Université and CNRS/IN2P3, Marseille, France

j Also at Universita di Napoli Parthenope, Naples, Italy

${ }^{\mathrm{k}}$ Also at Institute of Particle Physics (IPP), Victoria, Canada

${ }^{1}$ Also at Particle Physics Department, Rutherford Appleton Laboratory, Didcot, UK

${ }^{m}$ Also at Department of Physics, St. Petersburg State Polytechnical University, St. Petersburg, Russia

${ }^{\mathrm{n}}$ Also at Louisiana Tech University, Ruston, LA, USA

${ }^{\circ}$ Also at Institucio Catalana de Recerca i Estudis Avancats, ICREA, Barcelona, Spain

p Also at Graduate School of Science, Osaka University, Osaka, Japan

${ }^{q}$ Also at Department of Physics, National Tsing Hua University, Hsinchu City, Taiwan

${ }^{\mathrm{r}}$ Also at Department of Physics, The University of Texas at Austin, Austin, TX, USA

s Also at Institute of Theoretical Physics, Ilia State University, Tbilisi, Georgia

${ }^{t}$ Also at CERN, Geneva, Switzerland

"Also at Georgian Technical University (GTU), Tbilisi, Georgia

${ }^{v}$ Also at Manhattan College, New York, NY, USA

${ }^{w}$ Also at Hellenic Open University, Patras, Greece

${ }^{x}$ Also at Institute of Physics, Academia Sinica, Taipei, Taiwan

${ }^{y}$ Also at LAL, Université Paris-Sud and CNRS/IN2P3, Orsay, France

${ }^{\mathrm{z}}$ Also at Academia Sinica Grid Computing, Institute of Physics, Academia Sinica, Taipei, Taiwan

aa Also at School of Physics, Shandong University, Shandong, China

${ }^{a b}$ Also at Moscow Institute of Physics and Technology State University, Dolgoprudny, Russia

ac Also at Section de Physique, Université de Genève, Geneva, Switzerland

${ }^{\text {ad }}$ Also at International School for Advanced Studies (SISSA), Trieste, Italy

${ }^{\text {ae }}$ Also at Department of Physics and Astronomy, University of South Carolina, Columbia, SC, USA

${ }_{\text {af }}$ Also at School of Physics and Engineering, Sun Yat-sen University, Guangzhou, China

ag Also at Faculty of Physics, M.V. Lomonosov Moscow State University, Moscow, Russia

ah Also at National Research Nuclear University MEPhI, Moscow, Russia

ai Also at Department of Physics, Stanford University, Stanford, CA, USA

aj Also at Institute for Particle and Nuclear Physics, Wigner Research Centre for Physics, Budapest, Hungary

${ }^{\text {ak }}$ Also at University of Malaya, Department of Physics, Kuala Lumpur, Malaysia

* Deceased 